\title{
Early star catalogues of the southern sky ${ }^{\star}$ De Houtman, Kepler (Second and Third Classes), and Halley
}

\author{
Frank Verbunt ${ }^{1,2}$ and Robert H. van Gent ${ }^{3,4}$
}

\begin{abstract}
1 Astronomical Institute, Utrecht University, PO Box 80 000, 3508 TA Utrecht, The Netherlands; e-mail: f.w.m.verbunt@uu.nl 2 SRON Netherlands Institute for Space Research, Sorbonnelaan 2, 3584 CA Utrecht, The Netherlands

3 until Jan 2010: URU-Explokart, Faculty of Geosciences, Utrecht University, PO Box 80 115, 3508 TC Utrecht, The Netherlands

${ }^{4}$ Institute for the History and Foundations of Science, PO Box 80000, 3508 TA Utrecht, The Netherlands; e-mail: r.h.vangent@uu.nl
\end{abstract}

Received February 27, 2011 / Accepted April 5, 2011

\begin{abstract}
De Houtman in 1603, Kepler in 1627 and Halley in 1679 published the earliest modern catalogues of the southern sky. We provide machine-readable versions of these catalogues, make some comparisons between them, and briefly discuss their accuracy on the basis of comparison with data from the modern Hipparcos Catalogue. We also compare our results for De Houtman with those by Knobel (1917) finding good overall agreement. About half of the 200 new stars (with respect to Ptolemaios) added by De Houtman are in twelve new constellations, half in old constellations like Centaurus, Lupus and Argo. The right ascensions and declinations given by De Houtman have error distributions with widths of about $40^{\prime}$, the longitudes and latitudes given by Kepler have error distributions with widths of about $45^{\prime}$. Halley improves on this by more than an order of magnitude to widths of about $3^{\prime}$, and all entries in his catalogue can be identified. The measurement errors of Halley are due to a systematic deviation of his sextant (increasing with angle to $2^{\prime}$ at $60^{\circ}$ ) and random errors of 0.7 . The position errors in the catalogue of Halley are dominated by the position errors in the reference stars, which he took from Brahe.
\end{abstract}

Key words. astrometry - history and philosophy of astronomy

\section{Introduction}

The star catalogues of Tycho Brahe - available first in manuscript and then in print (Brahe 1598, 1602) - set a new standard of accuracy for positional astronomy. Brahe made his measurements at the island of Hven, at a latitude of $55^{\circ} 52^{\prime}$, and his catalogue accordingly was limited to declinations $\delta(1601) \gtrsim$ $-30^{\circ}$. Stars further south were known from Ptolemaios, who lived in Alexandria, at a latitude of $31^{\circ} 12^{\prime}$. An area around the southern equatorial pole, $\delta(150) \lesssim-53^{\circ}$, was wholly unknown to ancient Greek astronomers and Brahe's European contemporaries alike. In the course of the 17th century four catalogues were published to remedy this situation, by De Houtman (1603), Kepler (1627, two catalogues), and Halley (1679).

In 1603 Frederick de Houtman published a catalogue of 'many fixed stars, located around the south pole, never seen before this time', as he phrases it (in Dutch). De Houtman had sailed in 1595 with the first voyage by Dutch merchants from Amsterdam to Java and Sumatra, which is known in Dutch history as de Eerste Schipvaert (the first sailing of ships) to the Far East. De Houtman returned to Amsterdan in 1597, left in 1598 on a second voyage by competing merchants from Middelburg, was taken prisoner by the Sultan of Atjeh, was released in 1601, and returned to Amsterdam in July 1602. (Further details are given by Dekker 1987). In the preface to the catalogue, de Houtman states that it is based on his own measurements during

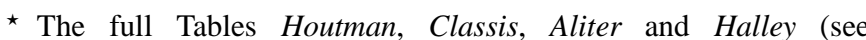
Tables 6. 7. 8) are available in electronic form only at the CDS via anonymous ftp to cdsarc.u-strasbg.fr (130.79.128.5) or via http://cdsweb.u-strasbg.fr/cgi-bin/qcat?J/A+A/ these voyages, upon which he improved while at Atjeh (Northern Sumatra). De Houtman distributed his stars over 21 constellations, among which 12 new ones (see Table 1).

These 12 new constellations had been delineated already in 1597 by Plancius based on positions of southern stars brought to Amsterdam with the returning Eerste Schipvaert. They were first shown on a globe made by Hondius (Van der Krogt 1993, p.152 sqq.). The measurements of the stars in the twelve new constelllations were ascribed on this globe to Pieter Dirksz Keyser - who died during the voyage. There has been some debate whether the catalogue by De Houtman is independent from the work by Keyser. We will return to this question in Section 5.4. De Houtman's catalogue was little known, perhaps not suprisingly as he published it as an appendix to a dictionary of the Malaysian and Madagaskar languages... However, his star positions were used on celestial globes made by Blaeu in 1603 and later (Dekker 1987).

A new edition of Brahe's star catalogue was edited in 1627 by Kepler, in his Tabulae Rudolphinae. To this catalogue Kepler appended two more, with stars which he decribed as belonging to the Secunda classis and Tertia classis (second and third class), respectively. He describes these classes in the beginning of each catalogue as follows (we translate from the Latin; words between [] are added).

[The second class] includes those fixed [stars] from the old catalogue of Hipparchos, retrieved and emended by Ptolemaios, that Tycho omitted. It is convenient to call them semi-Tychonian: indeed having sought them out from the [Greek] manuscript of Ptolemaios, and also 
Table 1. Constellations in the catalogue of De Houtman.

\begin{tabular}{rrrrrl} 
C & C $_{K}$ & & N & F & constellation \\
\hline 1 & 52 & Phe & 13 & 1 & Den voghel Fenicx \\
2 & 49 & CrA & 16 & 14 & De Zuyder Croon \\
3 & 38 & Eri & 7 & 30 & Het Zuyder eynde van den Nyli \\
4 & 62 & Hyi & 16 & 37 & De Waterslang \\
5 & 60 & Dor & 4 & 53 & Den Dorado \\
6 & $(40)$ & Col & 11 & 57 & De Duyve \\
7 & 42 & Arg & 56 & 68 & Argo Navis, het Schip \\
8 & 46 & Cen & 48 & 124 & Centaurus \\
9 & $(46)$ & Cru & 5 & 172 & De Cruzeiro \\
10 & 56 & Mus & 4 & 177 & De Vlieghe \\
11 & 59 & Vol & 5 & 181 & De Vliegende Visch \\
12 & 57 & Cha & 9 & 186 & Het Chameljoen \\
13 & 47 & Lup & 29 & 195 & Lupus, den Wolf \\
14 & 58 & TrA & 4 & 224 & Den Zuyder Trianghel \\
15 & 55 & Aps & 9 & 228 & De Paradijs Voghel \\
16 & 48 & Ara & 12 & 237 & Het Outaer \\
17 & 31 & Sco & 8 & 249 & De steert van Scorpio \\
18 & 54 & Pav & 19 & 257 & De Pauw \\
19 & 53 & Ind & 11 & 276 & De Indiaen \\
20 & 51 & Gru & 12 & 288 & Den Reygher \\
21 & 61 & Tuc & 6 & 299 & Den Indiaenschen Exster, \\
& & all & 304 & & \op Indies Lang ghenaemt
\end{tabular}

Notes. For each constellation the columns give the sequence number $C$, the sequence number $C_{\mathrm{K}}$ of the corresponding constellation in Kepler, the abbreviation we use, the number of stars in the constellation $N$, the sequence number of the first star in the constellation $F$, and the Dutch name as given by De Houtman. (The English translation of the constellation names is given by Knobel 1917). Brackets ( ) around $C_{K}$ indicate that the stars from the constellation in Houtman are listed in a different constellation in Secunda Classis. The twelve new constelllations are indicated with boldface.

${ }^{a}$ A ninth star is decribed ('the extreme one of the tail of Scorpio'), but no position is given; we omit it from Houtman.

using the [Latin] version of [George of] Trebizond published by Schreckenfuchs 76 years ago in Tübingen [actually Basel], I have converted them to the [end of the] year 1600, with the addition to the positions of the longitude recorded by Ptolemaios of such an angle as Tycho added to some other nearby bright [star]; and having added or subtracted to the latitude as much of the angle as at any place the obliquity under Ptolemaios is believed to have been greater; in any case in such a way that it would have the ratio of the adjacent round number.

Furthermore I have considered it expedient to follow this Greek text of Ptolemaios more closely, than among others the Prutentic, Copernican, and Alfonsine [texts], which appear to have followed the Arabic version of the Almagest; as in this way I would offer the opportunity to compare the versions among themselves, since it is uncertain whether the Arabs have corrected anything in these Ptolemaic [numbers], or whether all diversity among the versions originates from the inaccuracy of the copyists. There are a few, to which I have put my hand myself, in the book on the star in Serpentarius [i.e. SN1604], as well as others, which I have rendered in old character, to notify the reader of this.

The third class of fixed stars[:] comprising twelve celestial images, which can not be seen at all in our moderate northern zone. In his Uranometria Joh. Bayer reports that these have been observed by Amerigo Vespucci, Andreas Corsali, and Pedro de Medina, the
Table 2. Constellations in the Secunda Classis of Kepler

\begin{tabular}{|c|c|c|c|c|c|c|}
\hline $\mathrm{C}_{\mathrm{K}}$ & & $N$ & K & OT & constellation & Fig. \\
\hline 4 & Cep & 1 & 1005 & 12 & Cepheus & I-C.6 \\
\hline 5 & Boo & 1 & 1006 & 29 & Bootes & I-C.7 \\
\hline 7 & Her & 3 & 1007 & 29 & Hercules & I-C.9 \\
\hline 9 & Cyg & 1 & 1010 & 28 & Cygnus & I-C. 11 \\
\hline 11 & Per & 1 & 1011 & 34 & Perseus & I-C. 15 \\
\hline 13 & Oph & 26 & 1012 & 38 & Ophiuchus & C.21 \\
\hline 14 & Ser & 13 & 1038 & 14 & Serpens & C.21 \\
\hline 21 & And & 3 & 1051 & 24 & Andromeda & I-C. 27 \\
\hline 24 & Ari & 2 & 1054 & 22 & Aries & I-C.30,I-C. 45 \\
\hline 25 & Tau & 16 & 1056 & 44 & Taurus & C.22 \\
\hline 26 & Gem & 1 & 1072 & 30 & Gemini & I-C. 31 \\
\hline 27 & $\mathrm{Cnc}$ & 2 & 1073 & 16 & Cancer & I-C. 35 \\
\hline 29 & Vir & 2 & 1075 & 40 & Virgo & I-C. 37 \\
\hline 30 & Lib & 2 & 1077 & 19 & Libra & I-C. 38 \\
\hline 31 & Sco & 17 & 1079 & 11 & Scorpius & C.16 \\
\hline 32 & Sgr & 17 & 1096 & 30 & Sagittarius & C.24 \\
\hline 34 & Aqr & 4 & 1113 & 42 & Aquarius & I-C. 43 \\
\hline 35 & Psc & 6 & 1117 & 37 & Pisces & I-C. 44 \\
\hline 36 & Cet & 4 & 1123 & 22 & Cetus & I-C. 45 \\
\hline 38 & Eri & 20 & 1127 & 20 & Eridanus & C.25 \\
\hline 40 & $\mathrm{CMa}$ & 16 & 1147 & 14 & Canis Maior & $\overline{C .6}$ \\
\hline 42 & Arg & 42 & 1163 & 12 & Argo & C.7 \\
\hline 43 & Hya & 9 & 1205 & 25 & Hydra & C.26 \\
\hline 46 & Cen & 33 & 1214 & 5 & Centaurus & C.8 \\
\hline 47 & Lup & 19 & 1247 & 1 & Lupus & C.12 \\
\hline 48 & Ara & 7 & 1266 & 1 & Ara & C.15 \\
\hline 49 & $\mathrm{CrA}$ & 13 & 1273 & 1 & Corona Australis & C.2 \\
\hline 50 & PsA & 17 & 1286 & 1 & Piscis Austrinus & C.27 \\
\hline & , & 298 & & & & \\
\hline
\end{tabular}

Notes. For each constellation the columns give the sequence number $\mathrm{C}_{\mathrm{K}}$ (in KeplerE for $\mathrm{C}_{\mathrm{K}}<46$ ), its abbreviation, the number $N$ of stars added in Secunda Classis to KeplerE, and for the first star in the constellation the sequence number in the whole catalogue $\mathrm{K}$ and the sequence number within the constellation OT. Note that the numbers $C_{K}(\geq 46), K$ and OT are continued from KeplerE. We also give the Figure in which the new stars are depicted, where prefix 'I-' refers to Paper I.

first among Europeans, and declares that they were for the first time corrected to astronomical standard by Pieter Dicksz. [Keyser]. Jacobus Bartsch from Lausitz, a diligent young man, famous for some time now for his great merits concerning the celestial globe, assembled these same [constellations] into numbers and a map from the last tables and manuscripts of Johann Bayer himself (a splendid little collection of Christian constellations extracted from the Uranographia of Schiller, the publication of which is forthcoming in accordance with the last will of the author); and he has promised that he will subsequently publish the most perfect maps, by producing a one-an-a-half foot globe with the ancient images, as more conform with the version of Tycho.

Uranographia of Schiller refers to his celestial atlas, the Coelum Stellatum Christianum of 1627. Kepler thus put the stars known from Ptolemaios, but with positions more-or-less improved by comparison with improved positions of nearby stars, and corrected for the different value of the obliquity used by Ptolemaios, in his second class, together with some new stars measured by himself. In the third class he puts the stars of the new constellations. Kepler states that the positions of the third class are based on those of Petrus Theodorus, i.e. Pieter Dirksz. [Keyser], through the intermediary Bartsch. 
Table 3. Constellations in the Tertia Classis of Kepler

\begin{tabular}{rrrrll|}
$\mathrm{C}_{\mathrm{K}}$ & & $N$ & $\mathrm{~K}$ & constellation & Fig. \\
\hline 51 & Gru & 13 & 1303 & Grus & C.19 \\
52 & Phe & 15 & 1316 & Phoenix & C.1 \\
53 & Ind & 12 & 1331 & Indus & $\overline{\mid C .18}$ \\
54 & Pav & 23 & 1343 & Pavo & $\overline{\mid C .14}$ \\
55 & Aps & 11 & 1366 & Apus, Avis Indica & C.9 \\
56 & Mus & 4 & 1377 & Apis, Musca & C.11 \\
57 & Cha & 10 & 1381 & Chamaeleon & C.13 \\
58 & TrA & 5 & 1391 & Triangulum Aus. & C.10 \\
59 & Vol & 7 & 1396 & Piscis Volans, Passer & C.4 \\
60 & Dor & 7 & 1403 & Dorado, Xiphias & C.20 \\
61 & Tuc & 8 & 1410 & Toucan, Anser Americanus & C.4 \\
62 & Hyi & 21 & 1418 & Hydrus & \\
all & 136 & & &
\end{tabular}

Notes. Columns as in Table 2, without the OT

To our knowledge, no analysis has ever been published of the Secunda Classis and/or Tertia Classis.

The third catalogue of the stars in the southern sky was published by Edmond Halley (1679), on the basis of the measurements he made during a year (roughly Feb 1677 to Feb 1678) on the island of St. Helena, latitude $-15^{\circ} 57^{\prime}$. As Halley explains in his introduction to the catalogue, persistent bad weather prevented him from making planetary observations to determine the obliquity, and therefore he decided to determine the positions of the southern stars from the angular distance to stars from the catalogue of Brahe. In doing so he kept the ecliptic positions as given by Brahe. The bad weather also forced Halley to forego observations of the faint stars in Piscis Austrinus and the stars in Indus, in favor of more important brighter stars in other constellations.

Halley was 20 years and a bachelor student when he left; upon his return, with a recommendation from king Charles II, he was granted a master degree. For further details we refer to the biography of Halley writen by Cook (1998). An analysis of the star catalogue of Halley has been published by Baily (1843).

In this paper, we describe machine-readable versions of the star catalogues of De Houtman, Kepler (second and third classes) and Halley. The machine-readable tables give our identifications with stars from the (modern) Hipparcos catalogue, and on the basis of these the accuracy of the positions and magnitudes tabulated in the old catalogues. For the catalogue of De Houtman we compare our identifications with those made by Knobel (1917).

In the following we refer to (our machine-readable versions of) the star catalogues of De Houtman (1603) as Houtman; and of Halley (1679) as Halley. An F or E number is the sequence number of a star in Houtman, and Halley, respectively. (These letters stand for Frederick and Edmond, respectively, and are used to avoid confusion beween Houtman, Halley, and the catalogue of Hevelius 1690.) Thus F 273 is the 273rd entry in Houtman, and E 55 the 55th entry in Halley. Our emended, machine-readable versions of Kepler's (1627) edition of the star catalogue of Brahe (Verbunt \& Van Gent 2010a, hereafter: Paper I) and of his Second and Third Classes are referred to as KeplerE, Secunda Classis and Tertia Classis, respectively. A Knumber refers to an entry in these catalogues, where we continue the numbering: K 1004 is the last star in KeplerE, K 1005 and K 1302 the first and last entry in Secunda Classis, and K 1303 the first entry in Tertia Classis. The sequence number within a constellation is indicated by a number following the abbreviated
Table 4. Constellations in Halley.

\begin{tabular}{|c|c|c|c|c|c|}
\hline $\mathrm{C}$ & & $N$ & $\mathrm{E}$ & constellation & Fig. \\
\hline 1 & Sco & 29 & 1 & Scorpius & D.1 \\
\hline 2 & Sgr & 21 & 30 & Sagittarius & D.2 \\
\hline 3 & Eri & 30 & 51 & Eridanus & D.3 \\
\hline 4 & $\mathrm{CMa}$ & 5 & 81 & Canis Maior & D.4 \\
\hline 5 & PsA & 1 & 86 & Piscis Austrinus & D.5 \\
\hline 6 & Col & 10 & 87 & Colomba Noachi & D.6 \\
\hline 7 & Arg & 46 & 97 & Argo Navis & D.7 \\
\hline 8 & $\mathrm{RCa}$ & 12 & 143 & Robur Carolinum & D.8 \\
\hline 9 & Hya & 5 & 155 & Hydra & D.9 \\
\hline 10 & Cen & 35 & 160 & Centaurus & D.10 \\
\hline 11 & Lup & 23 & 195 & Lupus & $\mathrm{D.11}$ \\
\hline 12 & Ara & 9 & 218 & Ara, Thuribulum & $\mathrm{D} .12$ \\
\hline 13 & $\mathrm{CrA}$ & 12 & 227 & Corona Australis & $\mathrm{D} .13$ \\
\hline 14 & Gru & 13 & 239 & Grus & $\mathrm{D} .14$ \\
\hline 15 & Phe & 13 & 252 & Phoenix & D.16 \\
\hline 16 & Pav & 14 & 265 & Pavo & D.15 \\
\hline 17 & Aps & 11 & 279 & Apus Avis, Inidica & $\mathrm{D} .18$ \\
\hline 18 & Mus & 4 & 290 & Musca Apis & D.17 \\
\hline 19 & Cha & 10 & 294 & Chamaeleon & $\mathrm{D} .19$ \\
\hline 20 & $\operatorname{Tr} \mathrm{A}$ & 5 & 304 & Triangulum Australe & D.20 \\
\hline 21 & Vol & 8 & 309 & Piscis Volans & $\mathrm{D} .21$ \\
\hline 22 & Dor & 6 & 317 & Dorado, Xiphias & D.23 \\
\hline 23 & Tuc & 9 & 323 & Toucan, Anser Americanus & D.24 \\
\hline 24 & Hyi & 10 & 332 & Hydrus & $\mathrm{D} .22$ \\
\hline & & 341 & & & \\
\hline
\end{tabular}

Notes. For each constellation the columns give the sequence number $C$, the abbreviation we use, the number of stars in the constellation $N$, the sequence number of the first star in the constellation $E$, the constellation name and the Figure where it is shown. Due to bad weather, Halley observed only 1 star in Piscis Austrinus, and none in Indus.

name of the constellation: thus Phe 3 in the third star in Phoenix, in the catalogue under discussion.

We follow Hevelius (1690) in continuing the sequence numbering within each constelllation between KeplerE and Secunda Classis, indicated with OT (Ordo Tychonis) in the Tables (see also Verbunt \& Van Gent 2010b). Thus Cep 11 is the last star of Cepheus in KeplerE, to which Cep 12 is added in Secunda Classis.

\section{Description of the catalogues}

All catalogues are organized by constellation.

\subsection{Houtman (1603)}

The star catalogue of De Houtman (1603) contains 21 constellations, of which twelve are new, 8 are from Ptolemaios, and one, Cruzeiro, is the Southern Cross, split off from Centaurus. Table 1 lists the constellations and the number of stars in them. Each constellation starts with a statement of how many stars it contains, followed by a table with sequence number within the constellation, a short description of the star followed by five columns of integer positive numbers. The first two numbers give the right ascension in degrees $(G)$ and minutes $(M)$, the next two the declination in degrees $(G)$ and minutes $(M)$, and the fifth the magnitude of the star. Zeros are indicated either by an empty slot, or explicitly with 0 . The coordinates then are

$$
\alpha=G+M / 60 ; \quad \delta=-(G+M / 60)
$$

i.e. the minus-sign of the declination is not given in the tables but implicitly assumed. 
Interestingly, Houtman gives the coordinates in the equatorial system. The equinox of the catalogue is not given. We assume it is the same as that of KeplerE, i.e. AD 1601.0, JD 2305824.

\subsection{Secunda Classis, Tertia Classis, Kepler (1627)}

The star catalogue given as the Secunda Classis (second class) by Kepler (1627) starts with stars to be added to the 46 constellations listed in KeplerE, and ends with four constellations that were listed by Ptolemaios but which are too far south to be in KeplerE. Table 2 lists the constellations and the numbers of stars added to them with respect to KeplerE. For each entry a Latin description of the star is followed by the ecliptic longitude and latitude, and the magnitude. The longitude is given in integer degrees $(G)$, integer minutes $(M)$ and zodiacal sign $(Z)$. For one entry, K $1059, \frac{1}{2}$ is indicated behind the integer $M$. The longitude in decimal degrees thus is

$$
\lambda=(Z-1) * 30+G+M / 60
$$

The latitude is given in integer degrees $(G)$, integer minutes $(M)$ and B (for borealis, north) or A (australis, south). Often the B or A is not given explicitly, but implicitly taken to be the same as for the previous entry. The latitude in decimal degrees thus is:

$$
\beta= \pm(G+M / 60) ; \quad+/- \text { for } \mathrm{B} / \mathrm{A}
$$

Kepler gives a number of alternative positions in the Secunda Classis, in text between the catalogue entries, and sometimes in the entry lines themselves, usuallly as aliter (alternatively), in two cases (K 1066, K 1067) as Schreckenf (Schreckenfuchs). In the entry lines, the number for which a variant is given is marked by an explicit degree or arcminute sign.

The star catalogue given as the Tertia Classis (third class) by Kepler (1627) has the same layout as the second class. The constellations in it are the twelve new constellations delineated by Plancius. Table 3 lists the constellations in this catalogue and the number of stars in each of them.

Since Secunda Classis and Tertia Classis immediately follow the star catalogue of Brahe in Kepler (1627), we assume that they have the same equinox as KeplerE.

\subsection{Halley(1679)}

The catalogue of the southern stars, or supplement to the Tychonian catalogue of Halley (1679) is given in six columns. The first column contains the descriptions of the stars. The second and third column are headed unde observata (from where observed) and Distantia observata (observed distance), and contain the names of two reference stars for each entry and the angular distance of the entry to each of them, in degrees (gr.), minutes (m.) and seconds (s.), all in integers. For most entries two reference stars with angular distance are given, for some entries three or four reference stars; occasionally just one reference star is given, together with an indication that a longitude or latitude of the entry was used as given by Brahe or Kepler. The fourth column has two lines per entry, the first one giving the ecliptic longitude in zodiacal sign, degrees and minutes, and the second one the ecliptic latitude in degrees and minutes. An $s$ (for semi) may follow the minute indicating an extra 0.5 (both for longitude and latitude). The latitudes are followed by $b$ (for borealis) if the star has a northern latitude; for the other entries a southern latitude is implictly assumed. The fifth column gives the longitude and latitude in the same notation (without the s's and
Table 5. Certain identifications with objects not in the Hipparcos catalogue

\begin{tabular}{ll} 
entry & identification \\
\hline K1009 & globular cluster M13 \\
K1036 & supernova 1604 (Kepler) \\
K1230 & globular cluster $\omega$ Cen \\
K1408 & Large Magellanic Cloud \\
K1433 & Small Magellanic Cloud \\
F46 & Small Magellanic Cloud \\
E20 & open cluster NGC 6231 \\
E29 & open cluster M7 \\
E146 & highly variable star $\eta$ Car \\
E180 & globular cluster $\omega$ Cen
\end{tabular}

b's) E Catal. vetusto (from the old catalogue). Halley explains in his introduction that with old catalogue he refers to Clavius, Commentaries on Sacrobosco's De Sphaera and Bartsch's excerpt of the Rudolfine Tables by Kepler. The sixth column gives the magnitude as an integer.

Halley notes in the catalogue that due to bad weather he did not observe the faint stars in Piscis Austrinus, nor the stars in Indus. The last six stars of Lupus (E 212 - E 217) he observed inter navigandum (while sailing), and for these no angles to reference stars are given, but only the rough positions.

The equinox of the catalogue is given by Halley as Annum Completum 1677, old style. We assume that this corresponds to 1678 Jan 1, JD 2333948.

\section{Identification procedure}

The procedure that we follow for the identification of the stars in the different catalogues is mutatis mutandis the same as the procedure we followed for the catalogue of Brahe, and we refer to Paper I for details. Briefly, we select all stars for the Hipparcos Catalogue with a Johnson visual magnitude brighter than 6.0, we correct their equatorial position for proper motion between the Hipparcos epoch 1991.25 and the equinox of the catalogue, and precess the resulting equatorial coordinates to the equinox of the catalogue. For Secunda Classis, Tertia Classis, and Halley, we convert the equatorial coordinates to ecliptic coordinates, using the obliquity appropriate for the catalogue equinox.

For each entry in the old catalogue we then find the nearest in terms of angular separation - counterpart in the Hipparcos Catalogue. Often, this star is designated a secure counterpart by us, and given an identification flag 1 . This flag is also used for certain identifications with objects not in the Hipparcos Catalogue (see Table 5). Occasionally, a star brighter than the nearest star is considered by us to be the secure counterpart, and we flag such an identification with 2 . This may happen when the brighter star is at a marginally larger angular distance, or when it is part of a recognizable pattern. Examples are F 7 in Phoenix (near the center in Fig.C.1), and many stars in the constellation Corona Australis, both in Houtman and in Secunda Classis (Fig.C.2). Identifications we consider plausible but uncertain are flagged 3, and cases where several equally plausible counterparts are found, are flagged 4. An unidentified catalogue entry is flagged 5. As we will see, all repeat entries in Secunda Classis except one are repeating entries from KeplerE - with slightly different position, i.e. they are repeat entries only if Secunda Classis and KeplerE are considered as one catalogue. We mark these, and the only repeat entry within Secunda Classis, with a 6. Tertia Classis and Houtman do not contain repeat entries. 


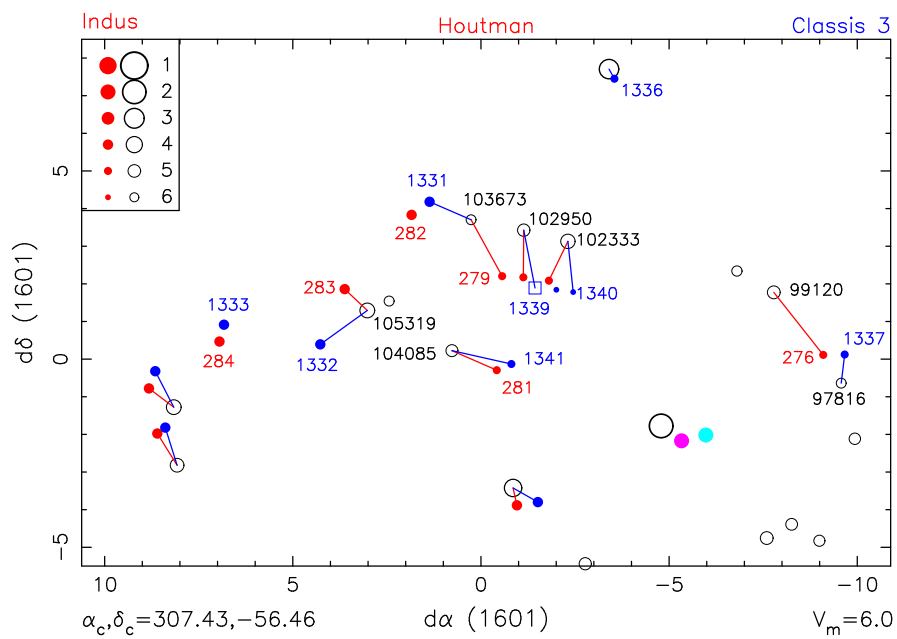

Fig. 1. Comparison of our identifications of entries in Indus in Houtman and Secunda Classis. In this projected image of the constellation Indus red stars and numbers refer to Houtman, blue to Secunda Classis and black to the Hipparcos Catalogue. The axes give the distances in right ascension and declination to the center of the constellation, roughly in degrees (for details of the projection used, see Sect.C).

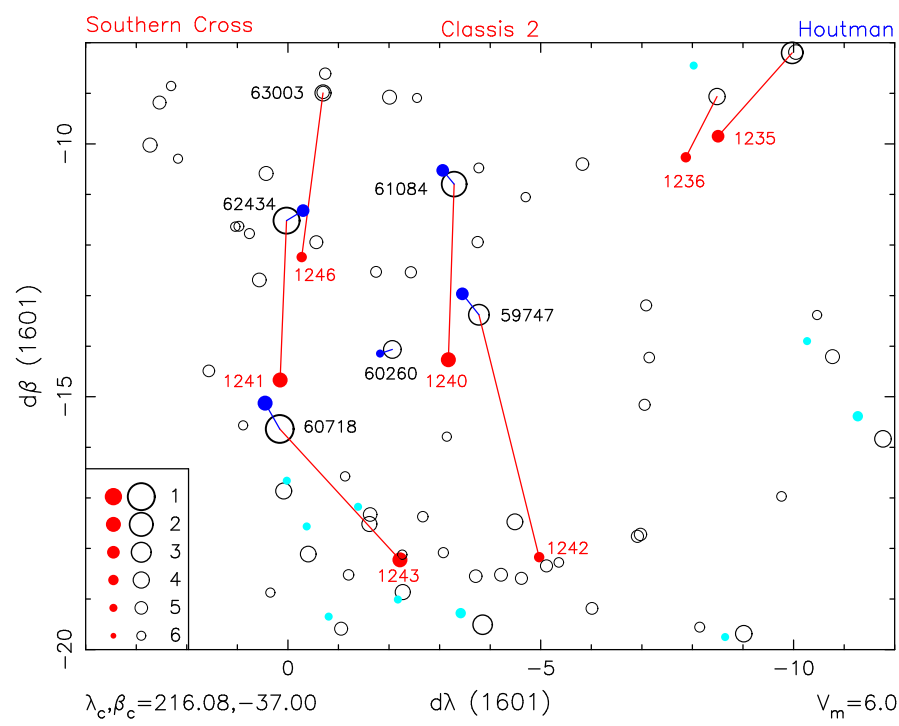

Fig. 2. Comparison of our identifications of entries in Crux in Secunda Classis and Houtman. In this projected image of the constellation Crux red stars and numbers refer to Secunda Classis, blue to Houtman and black to the Hipparcos Catalogue. The axes give the distances in ecliptic longitude and latitude to the center of the constellation, roughly in degrees (for details of the projection used, see Sect.C.

When no good Hipparcos star is found as identification, we investigate the possibility of extended sources such as star clusters, or extinct sources such as (super)novae. In a number of cases this leads to a good identification, as listed in Table 5 When a bright star is near or in a star cluster, the choice for single star or extended object is ambiguous. In two such cases, E 20 and E 29 we list the Hipparcos star in the catalogue, and the cluster in Table 5

In finalizing the identifications we have also compared our initial results for Secunda Classis and Tertia Classis with those for Houtman; this occasionally made us change an initial identi- fication. In crowded constellations we have limited a first search for counterparts to Hipparcos stars with $V<5.0$; this limits confusion by fainter and therefore less likely counterparts. In particular in Argo, many identifications flagged 2, i.e. the counterpart is not the nearest star with $V<6.0$, are the nearest Hipparcos star with $V<5.0$.

It may be argued that the majority of stars in Secunda Classis that are taken from the catalogue of Ptolemaios should properly be identified from that catalogue. We have in fact applied our identification procedure independently both to the Secunda Classis and to the catalogue of Ptolemaios, for which we use the edition by Toomer (1998). In almost all cases, the identifications are the same for corresponding entries in both catalogues; where they are not, they reflect the uncertainty in choosing between several plausible possibilities. We discuss this further in Sect.5.3.

To illustrate some of the problems encountered in trying to identify the catalogue entries, and our solutions of them, we discuss two of the more ambiguous cases. The first of these is the constellation Indus, illustrated in Fig.1 First, we note that with the sole exception of K 1336 ( $\alpha$ Ind) every entry in Tertia Classis is matched with an entry in Houtman. Thus, one may expect the same identification for each matched pair, and mostly this is indeed the case. An exception is the pair F 276/K 1337, where in the case of F 276 we choose the further but brighter HIP 99120 as counterpart and in the case of K 1337 the closer but fainter HIP 97816. We might have chosen to identify both F 274 and K 1339 with the same star, be it the fainter HIP 97816 or the brighter HIP 99120. Another exception involves HIP 102333, HIP 102950 and HIP 103673, three stars in a row. It is tempting to identify these with the three entries in a row about 1.5 to the south, and this we have done for the catalogue of De Houtman. This implies that F 282 is unidentified. In the case of Tertia Classis we have chosen to identify HIP 103673 with K 1331, and this implies that K 1338 - the star between K 1339 and K 1340 - remains unidentified. We have further chosen to identify F 283/K 1332 with HIP 105319. One could consider to identify K 1332 and K 1333 with HIP 104085 and HIP 105319 respectively, and leave K 1341 unidentified. We have chosen to identify K 1332 and K 1341 with HIP 105319 and HIP 104085, respectively, and leave K 1333 unidentified. The matching entries F 281, F 283 and F 284 suggest that this is the right choice. These examples show that a certain arbitrariness can not be avoided. Knobel (1917) made the same choices in identifying the entries in Indus in Houtman as we do.

As the second example we consider the Southern Cross, which in Secunda Classis is part of Centaurus (Fig.2). The identification of the stars in Houtman is straightforward, the identification of the stars in Secunda Classis far from straightforward. If we identify the bright entries K 1240 and K 1241 with HIP 61084 and HIP 62434, respectively, identification of K 1242 and K 1246 with HIP 59747 and HIP 63003 becomes plausible. What about K 1243 ? From its brightness, identification with HIP 60718 appears best, but from the direction of the offsets of the other identifications HIP 60260 may be possible as well. The positions as given by De Houtman for these stars are a marked improvement on (Kepler and through him on) Ptolemaios.

\subsection{Identifications of Houtman by Knobel}

Knobel (1917) identifies stars from Houtman with entries in the Uranometria Argentina (Gould 1879). We use the machinereadable version prepared by Pilcher (2010) which takes into account the (very few) corrections later made by Gould and others 
Table 6. First lines from the machine-readable table Houtman

\begin{tabular}{|c|c|c|c|c|c|c|c|c|c|c|c|c|c|c|c|}
\hline & $\mathrm{C}$ & & & $G$ & $M$ & $G$ & $M$ & $V_{F}$ & HIP & I & K & V & $\Delta \alpha$ & $\Delta \delta$ & $\Delta$ \\
\hline 1 & 1 & $=\mathrm{Phe}$ & 1 & 347 & 20 & 40 & 50 & 5 & 116231 & 1 & 1 & 4.4 & 29.6 & 49.1 & 54.1 \\
\hline 2 & 1 & $=\mathrm{Phe}$ & 2 & 348 & 35 & 44 & 15 & 5 & 389 & 1 & 3 & 4.7 & -15.5 & -33.7 & 35.5 \\
\hline 3 & 1 & $=$ Phe & 3 & 349 & 45 & 42 & 45 & 4 & 0 & 0 & 3 & 0.0 & 0.0 & 0.0 & 0.0 \\
\hline 4 & 1 & $=\mathrm{Phe}$ & 4 & 357 & 10 & 47 & 45 & 4 & 765 & 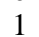 & 1 & 3.9 & 3.0 & -12.0 & 12.2 \\
\hline 5 & 1 & $=\mathrm{Phe}$ & 5 & 1 & 00 & 44 & 05 & 2 & 2081 & 1 & 1 & 2.4 & 34.7 & -24.0 & 34.6 \\
\hline 6 & 1 & $=\mathrm{Phe}$ & 6 & 1 & 30 & 45 & 34 & 4 & 2072 & 1 & 1 & 3.9 & 5.1 & -20.0 & 20.3 \\
\hline 7 & 1 & $=$ Phe & 7 & 2 & 40 & 50 & 15 & 4 & 2472 & 2 & 1 & 4.8 & 18.3 & -46.1 & 47.6 \\
\hline
\end{tabular}

Notes. For explanation of the columns see Sect.4.1.

Table 7. First lines from the machine-readable table Classis

\begin{tabular}{|c|c|c|c|c|c|c|c|c|c|c|c|c|c|c|c|c|c|c|}
\hline $\mathrm{K}$ & $C_{\mathrm{K}}$ & & & $Z$ & $G$ & $M$ & $G$ & $M$ & $S$ & $V_{K}$ & HIP & I & $V$ & $\Delta \lambda$ & $\Delta \beta$ & $\Delta$ & $\mathrm{P}$ & $\mathrm{I}_{\mathrm{P}}$ \\
\hline 1005 & 4 & $=\mathrm{Cep}$ & 12 & 1 & 04 & 30. & 64 & 00 & B & 5 & 107259 & 1 & 4.2 & -15.4 & 10.9 & 12.9 & 86 & 1 \\
\hline 1006 & 5 & $=$ Boo & 29 & 7 & 28 & 45. & 45 & 45 & B & 4 & 75312 & 6 & 5.0 & 165.0 & 65.6 & 131.5 & 91 & 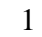 \\
\hline 1007 & 7 & $=$ Her & 29 & 9 & 01 & 20. & 55 & 55 & B & 5 & 83313 & 1 & 5.3 & 64.5 & 1.9 & 36.2 & 131 & 1 \\
\hline 1008 & 7 & $=$ Her & 30 & 9 & 02 & 30. & 58 & 15 & B & 5 & 83838 & 1 & 5.4 & 92.9 & 15.7 & 51.2 & 132 & 1 \\
\hline 1009 & 7 & $=$ Her & 31 & 8 & 24 & 39. & 57 & 30 & B & 5 & 0 & 1 & 5.8 & -40.9 & 23.8 & 32.3 & 0 & 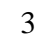 \\
\hline 1010 & 9 & $=\mathrm{Cyg}$ & 28 & 12 & 03 & 00. & 63 & 20 & B & 9 & 101138 & 2 & 4.9 & -147.3 & 43.6 & 78.5 & 175 & 1 \\
\hline
\end{tabular}

Notes. For explanation of the columns see Sect.4.2 The format of the file with alternative positions, Aliter, is identical

Table 8. First lines from the machine-readable table Halley

\begin{tabular}{|c|c|c|c|c|c|c|c|c|c|c|c|c|c|c|c|c|c|c|c|c|c|c|}
\hline$E$ & $C$ & & $Z$ & $G$ & $M$ & $G$ & $M$ & $V_{E}$ & HIP & $G$ & $M$ & $S$ & HIP & $G$ & $M$ & $S$ & HIP & $\mathrm{I}$ & V & $\Delta \lambda$ & $\Delta \beta$ & $\Delta$ \\
\hline 1 & $1=$ Sco & 1 & 8 & 28 & 41.0 & 01 & 5.0 & 2.5 & 65474 & 39 & 28 & 0 & 79593 & 16 & 15 & 20 & 78820 & 1 & 2.6 & 0.7 & -2.1 & 2.2 \\
\hline 2 & $1=\mathrm{Sco}$ & 2 & 8 & 28 & 4.0 & -01 & 54.0 & 2.5 & 65474 & 38 & 42 & 20 & 79593 & 19 & 13 & 10 & 78401 & 1 & 2.3 & 0.6 & -2.6 & 2.7 \\
\hline 3 & $1=\mathrm{Sco}$ & 3 & 8 & 28 & 26.0 & -05 & 23.0 & 3.0 & 65474 & 39 & 9 & 30 & 79593 & 22 & 42 & 10 & 78265 & 1 & 2.9 & 0.8 & -3.0 & 3.2 \\
\hline 4 & $1=\mathrm{Sco}$ & 4 & 8 & 28 & 38.5 & -08 & 28.0 & 3.5 & 65474 & 39 & 39 & 0 & 77070 & 35 & 44 & 0 & 78104 & 1 & 3.9 & 0.8 & -5.5 & 5.5 \\
\hline 5 & $1=\mathrm{Sco}$ & 5 & 9 & 00 & 8.0 & 01 & 42.0 & 4.0 & 65474 & 40 & 57 & 40 & 79593 & 15 & 46 & 40 & 79374 & 1 & 4.0 & 0.9 & -1.5 & 1.8 \\
\hline 6 & $1=\mathrm{Sco}$ & 6 & 8 & 29 & 11.0 & 00 & 16.0 & 5.0 & 65474 & 39 & 53 & 20 & 79593 & 17 & 4 & 20 & 78933 & 1 & 3.9 & -0.5 & -0.3 & 0.6 \\
\hline
\end{tabular}

Notes. For explanation of the columns see Sect.4.3.

to the 1879 version, and which adds among others the HD number of the stars. We convert this to the Hipparcos number, for comparison with our identification. (The Hipparcos Catalogue provides the HD number for each of its entries.) When the identification by Knobel is identical to ours we flag it $K=1$, when it is different from ours, we flag it 3 . (Flag 2, when we choose from two possible identifications the other one than he, does not occur.) Flag 0 indicates that Knobel has no identification.

\section{The machine-readable catalogues}

\subsection{The star catalogue of De Houtman}

The machine-readable table Houtman contains the following information (see Table 6). The first column gives the sequence number $\mathrm{F}$. The second and third column give the sequence number of the constellation $C$ and the abbreviation of the constellation name. The fourth column gives the sequence number within the constellation. Columns 5 and 6 give the right ascension in degrees $(G)$ and minutes $(M)$, and columns 7 and 8 the declination in degrees $(G)$ and minutes $(M)$. The negative declination (which all entries have) is implicit. Column 9 gives the magnitude according to De Houtman.

Columns 10-16 provide additional information from our analysis, viz. the Hipparcos number of our identification HIP, the flag I indicating the quality of the identification, the flag $\mathrm{K}$ which compares our identification with that by Knobel, the vi- sual (Johnson) magnitude $V$ given in the Hipparcos Catalogue for our identification, the differences in right ascension $\Delta \alpha$ and declination $\Delta \delta$ in minutes, and the angle $\Delta$ between the catalogue entry and our Hipparcos identification, in arcminutes ('). If the catalogue entry for minutes $M$ as given by De Houtman is $M_{\mathrm{F}}$ and the value computed from the position and proper motion in the Hipparcos Catalogue $M_{\mathrm{HIP}}$, then columns 14 and 15 give $M_{\mathrm{HIP}}-M_{\mathrm{F}}$.

\subsection{Secunda Classis and Tertia Classis from Kepler; variants to Secunda Classis}

The machine-readable table Classis combines the Secunda Classis and Tertia Classis. As noted above, Kepler gives alternative positions for some entries in Secunda Classis. Some of these alternative positions occur in KeplerE or its variants that we collected in Variants in Paper I. Such is the case for the alternative positions given for UMa 39, 40, 41 and 56 at the very beginning of Secunda Classis, and for Tau 30 (Electra in the Pleiades). Variants that do not occur in these earlier catalogues we collect in Aliter, with the exception of those for K 1139 and K 1140 , which we use to emend the main catalogue entry (see Sect.A.3.

The files Classis and Aliter contain the following information (see Table 7). The first column gives the sequence number $\mathrm{K}$. The second and third column give the sequence number of the constellation $C_{\mathrm{K}}$ and the abbreviation of the constellation name. 
The fourth column gives the sequence number within the constellation. Columns 5 - 7 give the longitude in zodiacal sign $(Z)$, degrees $(G)$ and minutes $(M)$, and columns 8 - 10 the latitude in degrees $(G)$, minutes $(M)$ and sign $S$. Column 11 gives the magnitude according to Kepler. A tabulated magnitude 8 indicates that Kepler does not give a magnitude; a 9 indicates a magnitude given by Kepler as ne(bulous).

Columns 12-18 provide additional information from our analysis, viz. the Hipparcos number of our identification HIP, the flag I indicating the quality of the identification, the visual (Johnson) magnitude $V$ given in the Hipparcos Catalogue for our identification, the differences in longitude $\Delta \lambda$ and latitude $\Delta \beta$ in minutes, and the angle $\Delta$ between the catalogue entry and our Hipparcos identification, in arcminutes ('). If the catalogue entry for minutes $M$ as given by Kepler is $M_{\mathrm{K}}$ and the value computed from the position and proper motion in the Hipparcos Catalogue $M_{\mathrm{HIP}}$, then columns 16 and 17 give $M_{\mathrm{HIP}}-M_{\mathrm{K}}$.

Columns 19 and 20 indicate the connection with the catalogue of Ptolemaios. Column 19 gives the sequence number $P$ of the entry in the catalogue of Ptolemaios which corresponds to the entry in Classis, in most cases based on a common Hipparcos identification, in which case column 20 contains a 1 . In some cases the correspondence is based on positional coicidence (after taking precession into account), but the identification is different: these have a 2 in column 20. This includes cases where one or both of the corresponding stars is unidentified. A zero in column 19 indicates that no corresponding entry is found, in these cases colum 20 has a 3, indicating a genuinely new entry.

\subsection{The catalogue of Halley}

The machine-readable table Halley uses one line for each 2-line entry in the catalogue as printed by Halley. Column 1 gives the sequence number for the entry in the catalogue as a whole $E$ (for Edmond), columns 2 and 3 the sequence number $C$ and abbreviation of the constellation, and column 4 the sequence number of the entry within the constellation. Columns 5-7 give the longitude in integers $Z$ and $G$ and real $M$, such that the longitude in decimal degrees is

$$
\lambda=(Z-1) * 30+G+M / 60
$$

. Columns 8-9 give the latitude with integer $G$ and real $M$ such that the latitude in decimal degrees is

$$
\beta= \pm(|G|+M / 60) ; \quad+/- \text { for } G>0 / G<0
$$

i.e. the sign of $G$ gives the sign of $\beta$. Column 10 gives the magnitude according to Halley.

Columns 11-14 give the Hipparcos number HIP of the first reference star and the angular distance of the entry to it in degrees $G$, arcminutes $M$ and arcseconds $S$, and columns 15-18 the same for the second reference star. In place of the distance to the second reference star a longitude or latitude from Brahe or Kepler is used in five cases; we indicate these with a negative value for HIP: -1 when a longitude from Brahe is used (for E 12), -2 latitude from Brahe (for E 9), -3 longitude from Kepler (for E 11, E 16 and E 17). Columns 15-20 give the results from our analysis, viz. the Hipparcos number of our identification, a flag $I$ indicating the quality of the identification, as explained in Sect.3 the magnitude of the Hipparcos entry of column 15 , the differences $\Delta \lambda \equiv \lambda_{\text {HIP }}-\lambda$ in minutes as tabulated and $\Delta \beta \equiv \beta_{\mathrm{HIP}}-\beta$ in arcminutes between the longitude $\lambda_{\text {HIP }}$ and latitude $\beta_{\text {HIP }}$ as derived from the Hipparcos catalogue and the values $\lambda$ and $\beta$ derived from the values in Halley, and the
Table 9. Frequency of identification flags in the four catalogues.

\begin{tabular}{l|rrrrr|r|r|r|r|r}
$\mathrm{I} \backslash \mathrm{K}$ & 0 & 1 & 2 & 3 & all & & $\mathrm{I}_{\mathrm{S}}$ & $\mathrm{I}_{\mathrm{T}}$ & & $\mathrm{I}_{\mathrm{E}}$ \\
\hline 1 & 3 & 185 & 0 & 19 & 207 & & 162 & 82 & & 336 \\
2 & 0 & 76 & 0 & 8 & 84 & & 71 & 48 & & 5 \\
3 & 0 & 4 & 0 & 4 & 8 & & 17 & 2 & & 0 \\
4 & 0 & 2 & 0 & 0 & 2 & & 0 & 1 & & 0 \\
5 & 2 & 0 & 0 & 1 & 3 & & 9 & 3 & & 0 \\
6 & 0 & 0 & 0 & 0 & 0 & & 39 & 0 & & 0 \\
all & 5 & 267 & 0 & 32 & 304 & & 298 & 136 & & 341
\end{tabular}

Notes. The distribution of the identification flags is given for Houtman (I), Secunda Classis $\left(\mathrm{I}_{\mathrm{S}}\right)$, Tertia Classis $\left(\mathrm{I}_{\mathrm{T}}\right)$, and Halley $\left(\mathrm{I}_{\mathrm{E}}\right)$. For Houtman we further give the distribution of the flags K comparing identifications by Knobel (1917) with ours. (The meaning of the flags is explained in Sect. 3)

distance $\Delta$ in arcminutes between the position derived from the Hipparcos catalogue and the position according to Halley.

The last four lines of the catalogue give additional angles to reference stars, with sequence number of star, sequence number and abbreviation of constellation, sequence number in constellation, and Hipparcos identification with flag, in columns 1-4 and 19, 20; and the Hipparcos number of the reference stars and the associated angles, in column 11-14 and 15-18.

\section{Analysis and discussion}

\subsection{The accuracy of Houtman}

Figure 3 and Table 9 display some results of our analysis of the star catalogue of De Houtman (1603). The catalogue consists of 304 entries, of which we claim to have identified 291 with certainty, one of which (F 46) with the Large Magellanic Cloud. In ten cases we suggest plausible or possible identifications, and in three cases we do not find a counterpart. Comparison with Classis suggests that the first of these unidentified entries, F 3 (near -9.9,6.5 in Fig.C.1), corresponds to K 1319 (near $-8.8,1.0$ ), but obtained a wrong position due to a computational or scribal error. The other two, in Indus, were discussed in Sect. 3 (see Fig.1).

As shown in Table 9, our identifications agree on the whole with those by Knobel (1917). We identify three of the five stars that Knobel (1917) left unidentified. In 27 cases we do not agree with the identification given by Knobel (1917) and give another identification; in 4 cases we prefer an alternative but consider his identification possible as well; and in the case of $\mathrm{F} 3$ comparison with Classis (see discussion in the previous paragraph and in Sect.(B) leads us to reject his identification.

The magnitude distributions of the 290 entries securely identified with a star in the Hipparcos Catalogue show a good correlation between the magnitudes as assigned by De Houtman and those of the counterparts in the Hipparcos Catalogue. The brightest category in Houtman is magnitude 1: all three stars in this category $(\alpha$ Eri $=$ Achernar, $\alpha$ Car $=$ Canopus, and $\beta$ Cen $)$ have $V_{\mathrm{HIP}}<0.5$. The faintest category in Houtman has magnitude 6 ; most stars in this category have $V_{\mathrm{HIP}}=5$.

The position errors $\Delta \alpha$ and $\Delta \delta$ are a mixture of random measurement errors and occasionaly large systematic errors, as illustrated by the Figures in Appendix C, e.g. Fig.C.2. As a result, the error distributions are not gaussians. Separate fits of gaussians to the central peak $\left(N>10\right.$ in error bins of $15^{\prime}$, as shown in Figure 3) and the whole distribution out to errors of $150^{\prime}$, we obtain $\sigma \sim 25^{\prime}$ and $\sigma \sim 40^{\prime}$, respectively. Since gaussians are 
Frank Verbunt and Robert H. van Gent: Early star catalogues of the southern sky
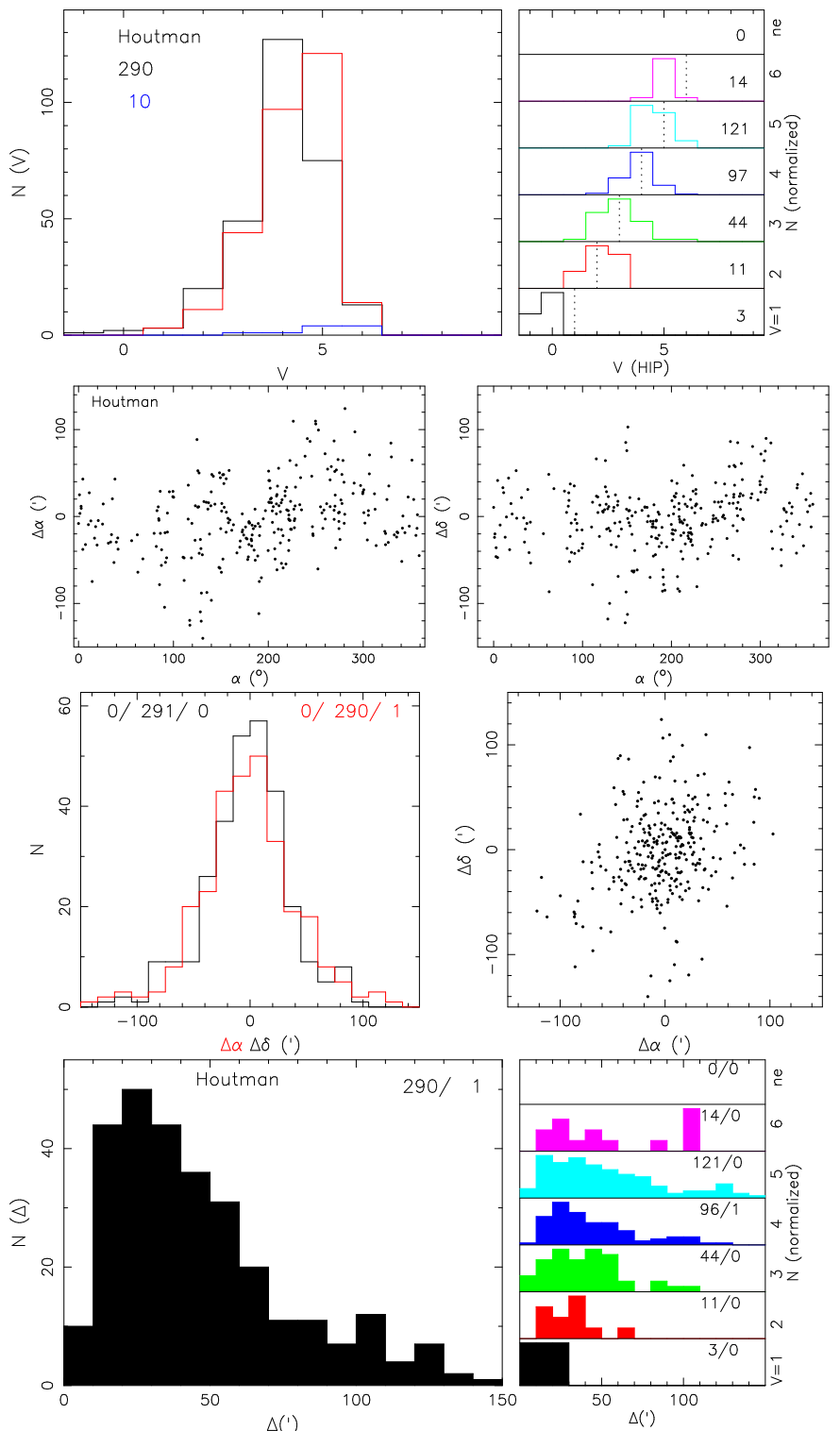

Fig. 3. Magnitude and position errors and their correlations in Houtman. The top frame shows the distributions of magnitudes according to Houtman for securely identified stars (identification flags 1,2; red) and for not securely identified stars (flags 3-5; blue), and of the magnitudes in the Hipparcos Catalogue for all securely identified stars (black) in the large frame, and for each magnitude according to Houtman separately in the small frames. The middle frames show the errors $\Delta \alpha$ in right ascension and $\Delta \delta$ in declination as a function of right ascension and of one another, and histograms of the position errors for the securely identified stars separately for $\Delta \alpha$ (red) and $\Delta \delta$ (black). The numbers indicate stars within the frame (middle) and outside the frame to the left or right. The lower frame shows the distributions of position error $\Delta$, for all securely identified sources in the large frame, and as a function of magnitude in Houtman in the small frames. The numbers indicate stars within/outside each frame.

not acceptable fits, these numbers should be considered as rough indicators only of the position accuracy. The errors in right ascension and in declination do not show clear correlations with right ascension or with one another. The distribution of the total position error $\Delta$ peaks near $25^{\prime}$, and shows a broad tail towards $\sim 100^{\prime}$, with larger errors for fainter magnitudes.
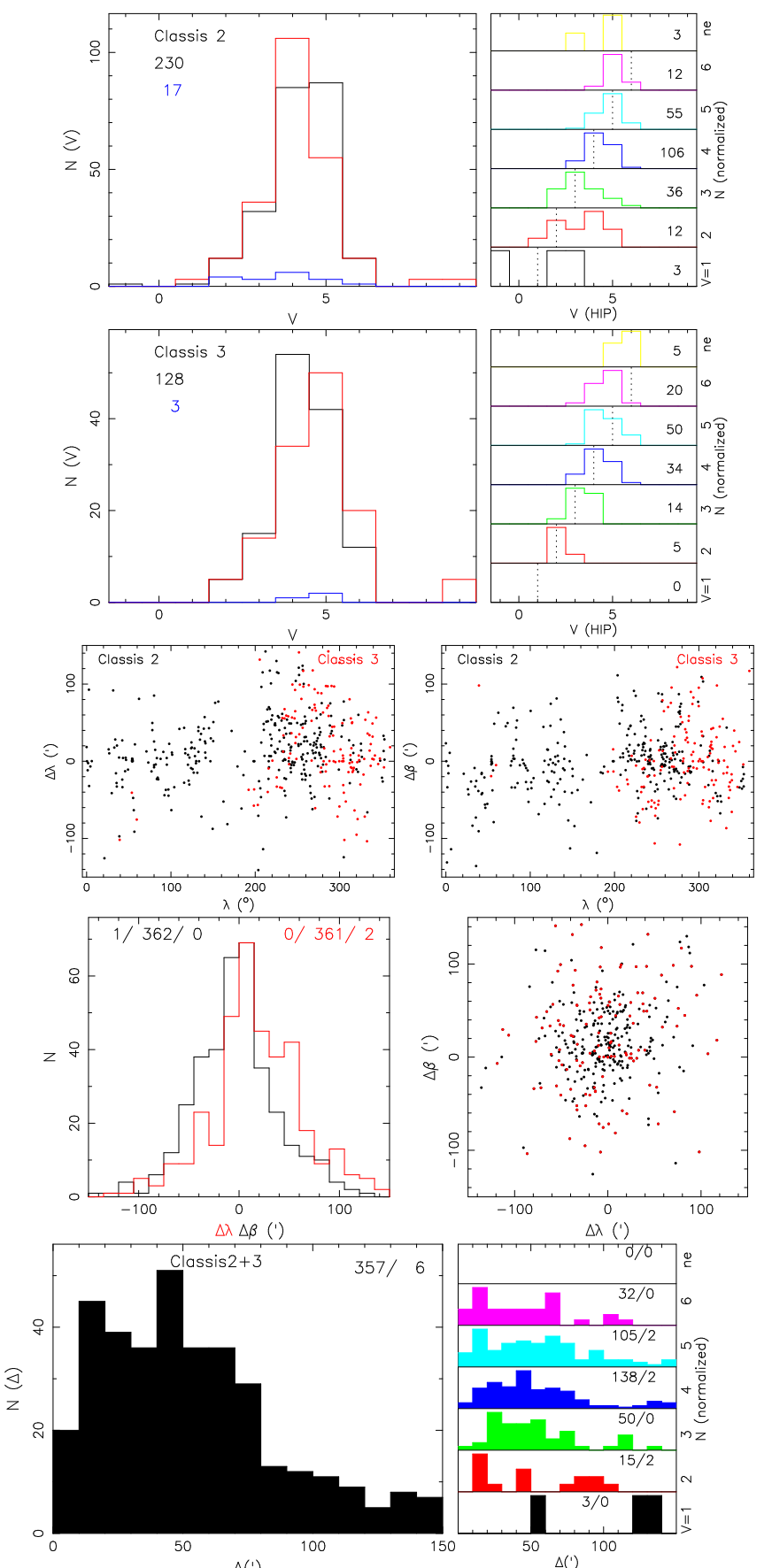

$\Delta\left({ }^{\prime}\right)$

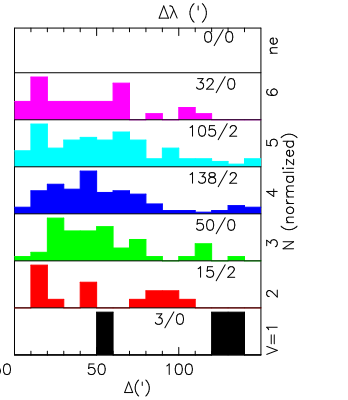

Fig. 4. Magnitude and position errors and their correlations in Secunda Classis and Tertia Classis. As Fig. 3, but with position errrors in the ecliptic system. In the middle correlation frames black/red dots indicate stars from Secunda Classis and Tertia Classis, respectively

\subsection{The accuracy of Secunda Classis and Tertia Classis}

Figure 4 and Table 9 display some results of our analysis of the second and third classes of Kepler (1627). The Table shows that Secunda Classis contains 39 repeat entries; all except one correspond to entries from KeplerE (Table 10). K 1296 repeats (or foreshadows) K 1303 in Tertia Classis. With few exceptions, the repeat entries in Secunda Classis have rather less accurate positions than the corresponding entries in KeplerE. Among the exceptions are some southern stars in Ophiuchus (Fig.C.21). 
Table 10. Stars in Secunda Classis repeating a star in KeplerE.

\begin{tabular}{rrrrccr}
\multicolumn{3}{c}{ Secunda Classis } & \multicolumn{3}{c}{ KeplerE } \\
K & Con & $\Delta_{S}\left(^{\prime}\right)$ & HIP & \multicolumn{1}{c}{ K } & Con & $\Delta_{K}\left({ }^{\prime}\right)$ \\
\hline 1006 & Boo & 131.5 & 75312 & 143 & Boo & 2.8 \\
1012 & Oph & 10.4 & 78727 & 685 & Lib & 3.2 \\
1013 & Oph & 34.0 & 78207 & 684 & Lib & 2.6 \\
1014 & Oph & 19.5 & 77853 & 683 & Lib & 3.3 \\
1015 & Oph & 18.6 & 80628 & 348 & Oph & 3.9 \\
1023 & Oph & 25.1 & 86284 & 336 & Oph & 46.4 \\
1024 & Oph & 25.1 & 84893 & 341 & Oph & 55.7 \\
1027 & Oph & 5.1 & 84970 & 355 & Oph & 131.6 \\
1028 & Oph & 10.3 & 85340 & 356 & Oph & 86.7 \\
1029 & Oph & 22.0 & 85755 & 357 & Oph & 97.5 \\
1032 & Oph & 33.4 & 88149 & 349 & Oph & 3.3 \\
1033 & Oph & 23.2 & 88192 & 350 & Oph & 2.7 \\
1034 & Oph & 53.4 & 88601 & 352 & Oph & 4.9 \\
1035 & Oph & 41.1 & 88290 & 351 & Oph & 2.7 \\
1037 & Oph & 64.3 & 88771 & 342 & Oph & 4.4 \\
1038 & Ser & 15.9 & 77257 & 343 & Oph & 2.6 \\
1040 & Ser & 17.5 & 84880 & 344 & Oph & 5.9 \\
1041 & Ser & 24.3 & 86263 & 345 & Oph & 4.7 \\
1042 & Ser & 21.2 & 86565 & 346 & Oph & 2.8 \\
1054 & Ari & 31.6 & 13061 & 504 & Ari & 3.8 \\
1055 & Ari & 32.6 & 12828 & 823 & Cet & 13.3 \\
1057 & Tau & 4.1 & 17499 & 534 & Tau & 37.1 \\
1058 & Tau & 2.8 & 17702 & 536 & Tau & 1.5 \\
1059 & Tau & 3.3 & 17608 & 535 & Tau & 8.6 \\
1062 & Tau & 2.5 & 17847 & 537 & Tau & 2.5 \\
1063 & Tau & 56.3 & 16852 & 918 & Eri & 0.6 \\
1064 & Tau & 47.0 & 23497 & 522 & Tau & 35.8 \\
1068 & Tau & 69.7 & 26640 & 326 & Aur & 1.3 \\
1069 & Tau & 34.2 & 27468 & 327 & Aur & 0.9 \\
1070 & Tau & 36.1 & 27830 & 324 & Aur & 2.1 \\
1071 & Tau & 56.9 & 28237 & 325 & Aur & 2.0 \\
1072 & Gem & 6.0 & 29696 & 323 & Aur & 1.4 \\
1127 & Eri & 53.3 & 20507 & 917 & Eri & 2.2 \\
1128 & Eri & 120.1 & 19849 & 916 & Eri & 0.7 \\
1129 & Eri & 123.6 & 13701 & 911 & Eri & 0.6 \\
1148 & CMa & 38.5 & 31592 & 938 & CMA & 18.8 \\
1205 & Hya & 78.3 & 42313 & 980 & Hyd & 1.4 \\
1206 & Hya & 14.9 & 42799 & 963 & Hyd & 1.3 \\
Secunda Classis & & & Tertia Classis \\
1296 & PsA & 41.0 & 108085 & 1303 & Gru & 11.0
\end{tabular}

Notes. For each repeat star in Secunda Classis, the table gives the constellation and position error $\Delta_{s}$ to its identification HIP, and the sequence number and constellation of the corresponding entry in KeplerE with its position error $\Delta_{K}$. In one case the corresponding entry is in Tertia Classis

The three securely identified entries (flags 1,2 ) with no magnitude or a magnitude 'nebulous' in the upper small frame for Secunda Classis in Fig. 4 are K 1010 in Cygnus (possibly indicating a combination of two stars, see Sect.[B), K 1099 in Sagittarius (the close binary $v^{1}, v^{2}$ Sgr), and K 1093 a single star for which it is not clear why it would be marked nebulous. For three new stars of the Pleiades Secunda Classis gives no magnitude. The five securely identified (flags 1,2 ) stars with a magnitude 'nebulous' in the frame for Tertia Classis are K 1324 and K 1325 in Phoenix (Fig.C.1), K 1339 in Indus (Fig.11), and K 1349 and K 1351 in Pavo (Fig.C.17), for none of which there is an obvious reason for the label nebulous.

Separate fits of gaussians to the central peak of the distributions of errors $\Delta \lambda$ in longitude and $\Delta \beta$ in latitude $(N>10$ in error bins of $15^{\prime}$, as shown in Figure 4) and the whole distribution out to errors of $150^{\prime}$, give $\sigma \sim 35^{\prime}$ and $\sigma \sim 45^{\prime}$, respectively. Separate fits for Secunda Classis and Tertia Classis show that the errors in Secunda Classis are on average about 5' smaller; those in Tertia Classis are larger by up to $10^{\prime}$. However, as the errors are the sum of random and systematic errors, not well described by gaussian distributions, these numbers must be considered as rough indicators of the position accuracy only. The errors in longitude and in latitude do not show clear correlations with right ascension or with one another. The distribution of the total position error $\Delta$ has a broad peak from 20 to $70^{\prime}$, and a broad tail towards larger error. The importance of systematic errors causes the errors for bright stars to be similar to those of fainter stars.

\subsection{Comparison between Classis and the star catalogue of Ptolemaios}

To compare the stars in Classis with those in the star catalogue of Ptolemaios we use the edition of the latter by Toomer (1998). For this we convert the identifications given by Toomer to Hipparcos numbers; and precess the coordinates of the entries in Classis to the epoch of the catalogue of Ptolemaios. In doing so, we assume that the positions in the star catalogue of Ptolemaios, after subtraction from the longitude of $2^{\circ} 40^{\prime}$, correspond to the epoch of $-128(=129 \mathrm{BC})$, as stated by Ptolemaios in the Almagest (Toomer 1998, p.333). II In some cases the correspondence with Ptolemaios is explicit in the description of the entry in Classis. For example, K 1006 is described 10 Ptol. in Classis, and K 1040-1042 as 13-15 Ptol. In the other cases we determine corresponding pairs by their having the same counterpart from the Hipparcos Catalogue, and graphically by similar positions in the constelllation.

An example of the graphical comparison is given in Fig.5, in which we show the constellations of Ophiuchus and Serpens, the constellations to which Kepler added most new stars. Short solid lines connect the catalogue entries with the center of their Hipparcos identifications in this Figure, enabling for many Classis entries a straightforward determination whether they have a corresponding entry in the star catalogue of Ptolemaios. (Note that in some cases the entry from the Hipparcos catalogue is almost hidden behind the Classis entry in the Figure, viz. K 1043 and K 1019, near 3.8,-6.3 and -9.1,-13.4 in Fig.5])

Among the entries in Classis that are not matched in the star catalogue of Ptolemaios we note the globular clusters M 13 in Hercules (K 1009) and $\omega$ Cen, SN 1604 in Ophiuchus (K 1036), and four new stars in the Pleiades (K 1056 and K 1058-1060),

\subsection{Comparison between Houtman and Classis}

In Table 11 we compare the numbers of entries in those constellations that are listed both in Houtman and in Classis. and the numbers of matching pairs, i.e. entries in Houtman and Classis that are identified with the same star in the Hipparcos Catalogue. We see from the Table that 99 of the 112 entries in the twelve new constelllations in Houtman are matched by a star in Classis, all of them in Tertia Classis. To this may be added 8 matches not with the same Hipparcos identification, viz. F 3 (see Sect.B) in Phoenix, F 46 (the Small Magellanic Cloud), and three stars each

1 The longitudes thus found for the catalogue of Ptolemaios are more accurate than the tabulated longitudes for the epoch of Ptolemaios, 137 AD, which are systematically too low by about a degree. This suggests that Ptolemaios did not make his own measurements, but corrected the positions measured by Hipparchos with his estimate for the change due to precession, i.e. $2^{\circ} 40^{\prime}$; the correct value being $\simeq 3^{\circ} 40^{\prime}$. An ingenious analysis by Duke (2003) shows that Ptolemaios did indeed copy most of his star positions from Hipparchos. 


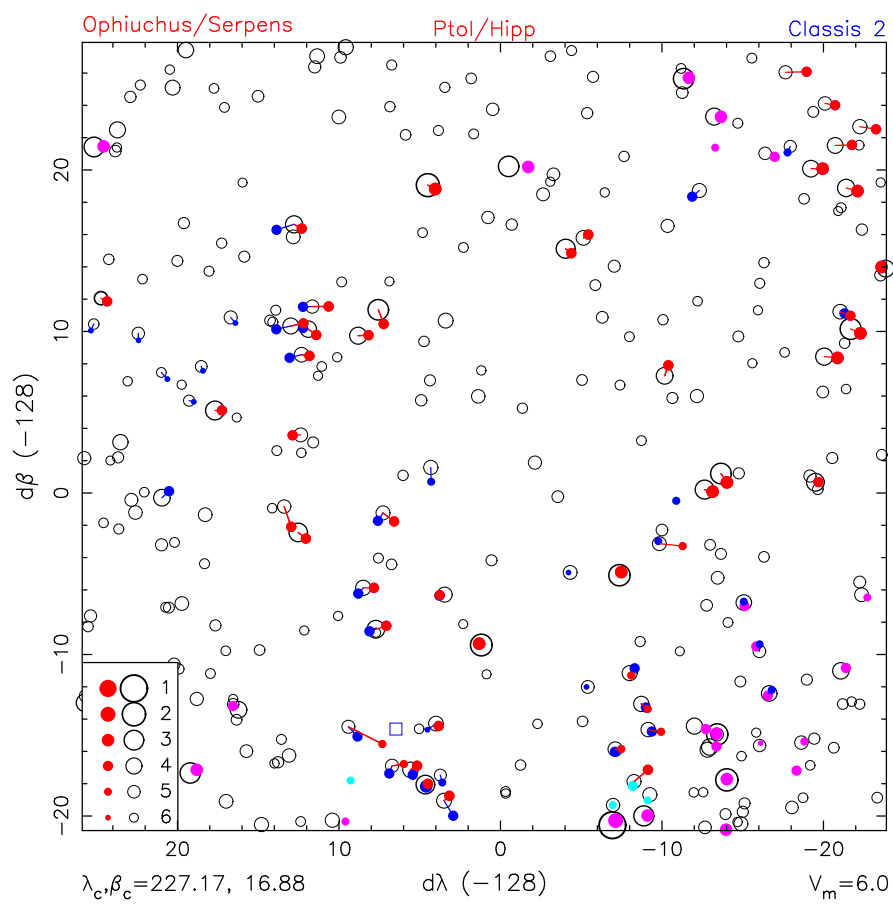

Fig. 5. The stars of Ophiuchus and Serpens in the star catalogue of Ptolemaios (red; other stars from this catalogue in purple) and in Secunda Classis after precession of the coordinates to the epoch of Ptolemaios/Hipparchos (dark blue; other stars in Classis in light blue). Open black circles are stars from the modern Hipparcos Catalogue. Short solid lines indicate identifications. In many cases stars in Classis are matched by an entry in the star catalogue of Ptolemaios, occasionally by an entry in a different constellation. New stars in Classis, not matched by an entry in Ptolemaios' catalogue are also seen, including the supernova of 1604 , indicated as a blue square near $6,-14$.

in Pavo and Indus whose pattern matches between Houtman and Classis, even though identified with different Hipparcos stars (Figs.1, C.17). Thus the new constellations as listed in Houtman contain only five entries not found in Classis. Conversely, Tertia Classis contains 29 stars not in Houtman.

In comparing constelllations from Ptolemaios in Houtman with Classis, we see that 101 entries in Houtman are identified with the same star in the Hipparcos Catalogue as a star in Classis, all in Secunda Classis. The pair F 63-K 1153 in Colomba / Canis Maior may also be considered a match even though they are identified with different Hipparcos stars (near 3, -0.5 in Fig.C.6. Thus there are 90 stars in these constellations in Houtman not matched in Classis. Four of these, the northernmost stars in Centaurus, are actually in KeplerE.

Knobel (1917) gives a table in which the numbers of stars new with respect to Ptolemaios are given, for each constellation separately. For the twelve new constellations, these numbers are virtually identical to the total number of stars in them; of the stars in Houtman but not in Tertia Classis, one star in Hydrus and one in Grus are considered by Knobel to be present in the catalogue of Ptolemaios (we find one in Grus, F 287=P 1022, but none in Hydrus). For the old constellations, Knobel's numbers are very close to the numbers of stars in each constellation in Houtman minus the numbers of matches with Secunda Classis. This is understandable, as Secunda Classis is mainly composed of stars from the catalogue of Ptolemaios (see previous Section). One star in Corona Austrinus, two in Centaurus/Crux (not counting
Table 11. Numbers of matching stars in Houtman and Tertia Classis and Secunda Classis.

\begin{tabular}{crrr|crrr} 
Con & H & $3 \mathrm{C}$ & $\mathrm{M}$ & Con & H & $2 \mathrm{C}$ & $\mathrm{M}$ \\
\hline Phe & 13 & 15 & $12+1$ & CrA & 16 & 13 & 11 \\
Hyi & 16 & 21 & $12+1$ & Eri & 7 & 20 & 1 \\
Dor & 4 & 7 & 3 & Col & 11 & 16 & $9+1 / 1$ \\
Mus & 4 & 4 & 4 & Arg & 56 & 42 & 22 \\
Vol & 5 & 7 & 5 & Cen & 53 & 33 & $27 / 1$ \\
Cha & 9 & 10 & 9 & Lup & 29 & 19 & 14 \\
TrA & 4 & 5 & 4 & Ara & 12 & 7 & $6 / 1$ \\
Aps & 9 & 11 & $7 / 2$ & Sco & 8 & 17 & 8 \\
Pav & 19 & 23 & $16+3$ & & & & \\
Ind & 11 & 12 & $8+3$ & & & & \\
Gru & 12 & 13 & 12 & & & & \\
Tuc & 6 & 8 & 5 & & & & \\
\hline tot & 112 & 136 & $99+8$ & tot & 192 & 167 & $101+1$
\end{tabular}

Notes. For each constellation the numbers of stars in Houtman and in Secunda Classis (2C) or Tertia Classis (3C) is given as well as the number of matching pairs. Most numbers indicates matches based on identifiction with the same star in the Hipparcos Catalogue. A match of a star in Houtman with a star in a different constellation in Classis is indicated behind a slash; Colomba and Canis Maior are treated as one constellation. The numbers behind a + sign indicate pairs that we consider matches in addition, even though identified with different Hipparcos stars, or unidentified.

the four matches with KeplerE), and one in Lupus, not matched in Secunda Classis, are considered by Knobel to be matched with stars from the catalogue of Ptolemaios.

Our analysis of Secunda Classis, Tertia Classis, and Houtman leads us to agree with the conclusions of Stein (1917) that the data for the twelve new constellations were mostly obtained during the Eerste Schipvaert, to which Keyser and De Houtman both contributed, and that the data for the new stars in the Ptolemaean constellations were obtained by De Houtman during his stay in Sumatra. Indeed, this agrees with the statement of De Houtman in his introduction to the star catalogue, except that he does not acknowledge a contribution by Keyser.

Knobel (1917) correctly notes that the twelve new constellations can not have been observed by De Houtman from Sumatra, but his conclusion that De Houtman merely plagiarized Keyser is not warranted. The fact that Secunda Classis has none of the stars added by Houtman to the Ptolemaean constellations, indicates that Kepler in 1627 used only data from the Eerste Schipvaert. The large similarity between the lists of stars in Tertia Classis with those for the twelve new constellations in Houtman then indicates a connection between the two viz. the observations obtained during the Eerste Schipvaert.

Dekker (1987) shows significant differences between the depictions of the twelve new constellations by Hondius on globes in 1598 and 1601 (and therefore based on data from the Eerste Schipvaert) and those by Blaeu on his globe of 1603 (based on data from De Houtman). Dekker concludes that Keyser and De Houtman recorded their observational data separately. The large overlap in the star lists lead us to suggest an alternative explanation, viz. independent reductions by Plancius and Blaeu of a shared set of observational data. The presence in Tertia Classis of 29 stars not in Houtman then shows that de Houtman did not use all stars from the shared set, possibly because he omitted the stars that he had not measured himself. 


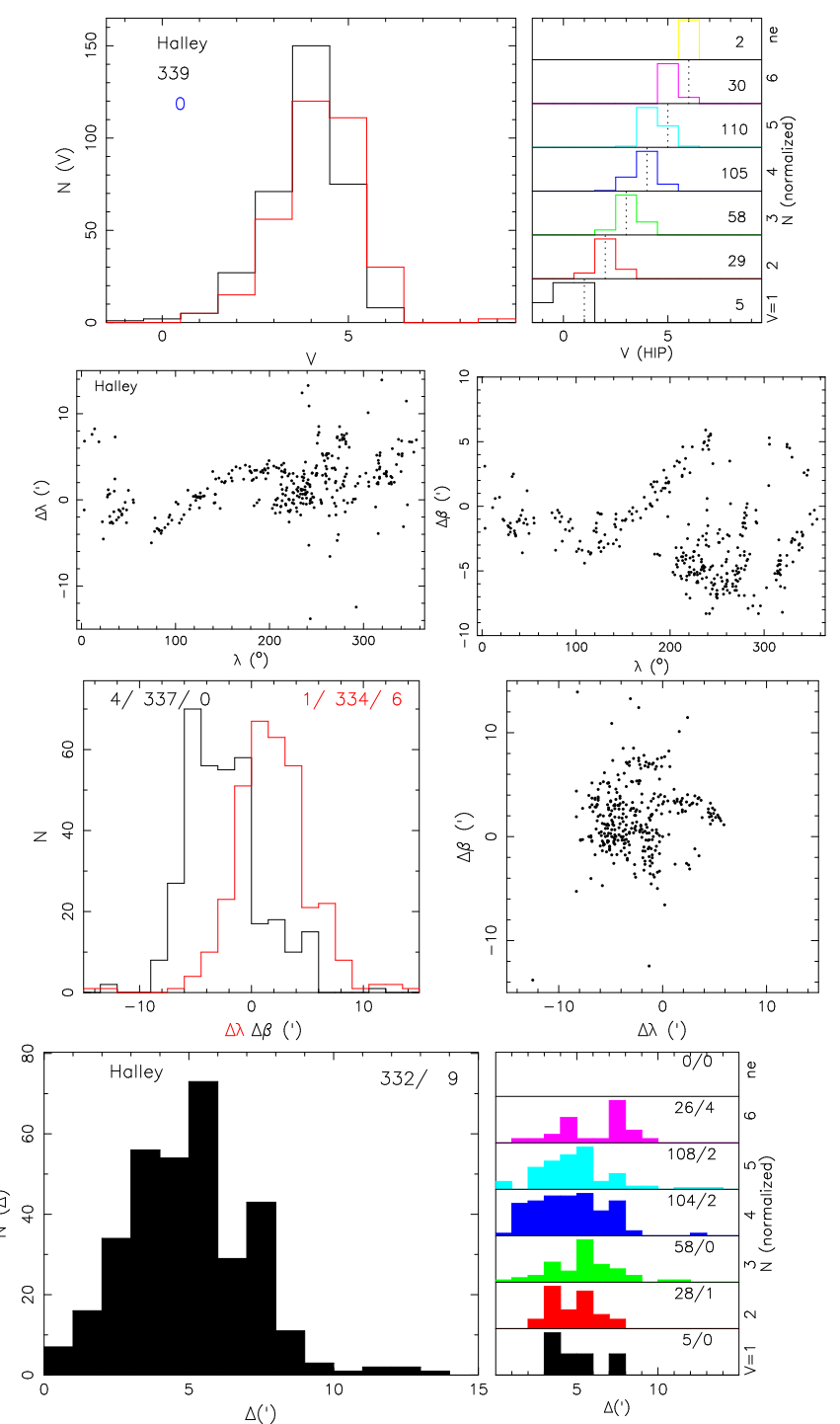

Fig. 6. Magnitude and position errors and their correlations in Halley. As Fig. 3, but with position errrors in the ecliptic system.

\subsection{The accuracy of Halley}

Figure 6 and Table 9 display some results of our analysis of the star catalogue of Halley (1679), and illustrate its remarkable accuracy. Separate fits of gaussians to the distributions of errors $\Delta \lambda$ in longitude and $\Delta \beta$ in latitude as shown in Figure 6 give $\sigma \sim 3^{\prime}$ for both, with offsets of $2^{\prime}$ for $\Delta \lambda$ and $-2.6^{\prime}$ for $\delta \beta$. These numbers justify what Halley writes in the introduction to the catalogue (we translate from the Latin):

Then there is a rumour that a certain Dutchman Frederick [de] Houtman has made an effort on these stars on the island of Sumatra, and that Willem Blaeu [used] his observations to correct the celestial globe which he [Blaeu] published. Which instruments he used is not known to me, but from a comparison made of his globe with our catalogue, it is sufficiently and abundantly clear that this observer was little practised in this arena.

This is somewhat harsh on De Houtman, who probably used an ordinary mariner's cross-staff, whereas Halley used an astronomical sextant with telescopic sights, made specifically for his observations in St. Helena, probably in the Ordnance Office (Cook, 1998, pp. 38).

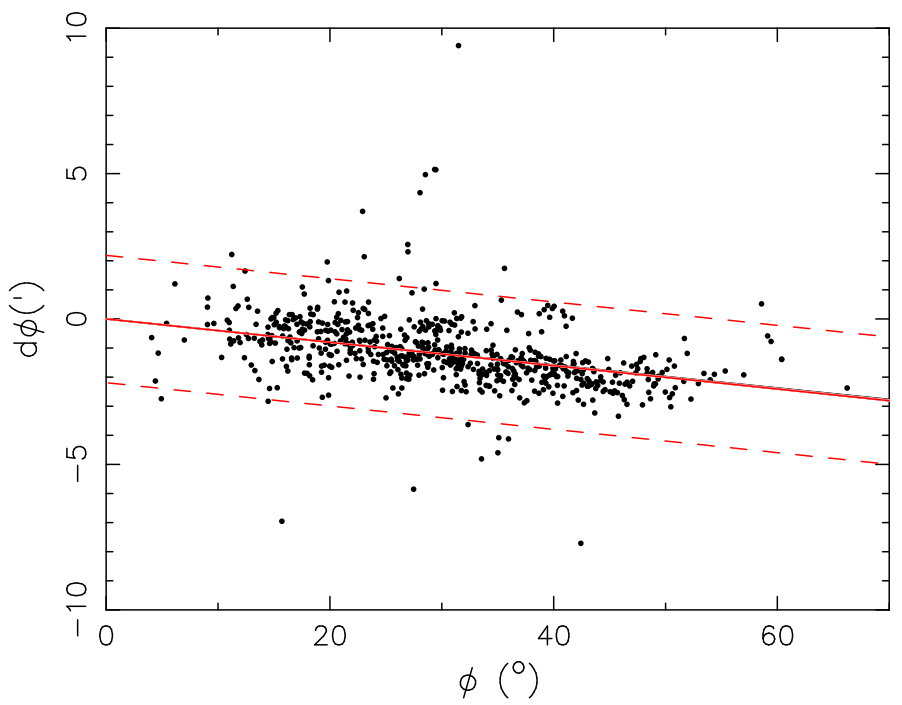

Fig. 7. For each angle $\phi$ between an entry in Halley and a reference star, the figures shows the difference $d \phi \equiv \phi_{E}-\phi$ between the catalogue value $\phi_{E}$ and the modern value $\phi$, as a function of $\phi$. The red line shows a linear fit, forced to go through $(0,0)$, in which points deviating more than 3-sigma have been excluded. The 3-sigma range is indicated with the dashed lines.

Fig. 6 shows that the correlations of the errors in longitude $\Delta \lambda$ and latitude $\Delta \beta$ with longitude $\lambda$ clearly are not random. (The largest errors, of three stars in Lupus, observed only from the ship while sailing, are outside the frames.) The position errors of the stars in Halley are the result of his own measurement errors and of the errors in the positions of the reference stars that he used, from the catalogue of Brahe. Our identifications of the reference stars are given in Table A.1. The angular distances given in the catalogue of each entry to the reference stars allow us to test the measurements errors of Halley, by comparing them with the values derived from the Hipparcos Catalogue, taking into account the proper motion between 1991.25 and 1678.0. The result is shown in Figure 7 The sextant shows a systematic error, increasing roughly linearly to about $2^{\prime}$ at the largest angles of about $60^{\circ}$, on which errors with a standard deviation $\sigma \simeq 0$ '. 7 are superposed.

Table A.1 shows that both the systematic errors and the random errors in the angles with the reference stars are small with respect to the position errors of many reference stars themselves, both of some primary references (e.g. $\sigma$ Sgr and Fomalhaut) and of secondary references derived from them. These errors explain both the range and the systematics seen in the error correlation plots in Fig. 6. Even so, it may be concluded that Halley achieved a stunning accuracy in his catalogue.

A problem of identification with earlier catalogues is mentioned by Halley following his list of the stars in Argo. He remarks that Ptolemaios assigns a magnitude 2 to the 31 st star in his list for the constellation of Argo, but that there is now little or no remanant of this star. Halley qualifies this by noting that in particular the southern stars in Argo have such large positional errors that identification with the stars in his catalogue cannot be be made safely. In fact, it appears to us that Halley's entry E 118 is a good match for Arg 31 of Ptolemaios: both entries have magnitude 2 in the old catalogues, and both are identified with HIP 44816 ( $\lambda$ Vel), a $V=2.2$ star.

Acknowledgements. We thank Jetze Touber and Rienk Vermij for help in translating the Latin texts heading Secunda Classis and Tertia Classis. This research 
has made use of the SIMBAD database, operated at CDS, Strasbourg, France, and was supported by the Netherlands Organisation for Scientific Research under grant 614.000.425.

\section{References}

Baily, F. 1843, Mem.R.A.S., 13, 35 and 13, 167

Brahe, T. 1598, Stellarum octavi orbis inerrantium accurata restitutio (manuscript), edited by Dreyer 1916, pp.333-373; pp.412-417

Brahe, T. 1602, Astronomiae Instauratae Progymnasmata edited by Dreyer 1915, pp. 258-280; pp.451-452

Cook, A. 1998, Edmond Halley, charting the heavens and the seas (Clarendon Press, Oxford)

Duke, D.W. 2003, JHA, 34, 227

de Houtman, F. 1603, Spraeck ende woord-boeck, inde Maleysch ende Madagaskarsche talen (Cloppenburch, Amsterdam)

Dekker, E. 1987, Annals of Science, 44, 439

Dreyer, J.L.E. 1915, Tychonis Brahe Dani Scripta Astronomica, Vol.II (Gyldendal, Kopenhagen)

Dreyer, J.L.E. 1916, Tychonis Brahe Dani Opera Omnia, Vol.III (Gyldendal, Kopenhagen)

ESA 1997, The Hipparcos and Tycho Catalogues (SP-1200, ESA Publications Division, Noordwijk)

Gould, B.A. 1879, Uranometria Argentina (Observatorio Nacional Argentino, Cordoba);

Halley, E. 1679, Catalogus stellarum australium (Thomas James \& R. Harford, London)

Hevelius, J. 1690, Catalogus Stellarum Fixarum (Johann Stolle, Gdansk)

Kepler, J. 1627, Tabulae Rudolphinae (Jonas Saur, Ulm)

Knobel, E.B. 1917, MNRAS, 77, 414 and 77, 580

Pilcher, F. 2010, Gould's (1917) Uranometria Argentina reprinted and updated http://vizier.u-strasbg.fr/viz-bin/Cat?V/135

Stein, J. 1917, Studiën, tijdschrift voor godsdienst, wetenschap en letteren, 88, 36

Toomer, G.J. 1998 Ptolemy's Almagest, 2nd ed. (Princeton University Press)

van der Krogt, P. 1993, Globi Neerlandici: the production of globes in the Low Countries (HES, Utrecht)

Verbunt, F., van Gent, R.H. 2010a, A\&A, 516, A28 (Paper I)

Verbunt, F., van Gent, R.H. 2010b, A\&A, 516, A29

\section{Appendix A: Annotations and Emendations}

\section{A.1. Emendations to Knobel}

Vel55 is identified by Knobel as $215=\mathrm{x}$ Vel from the Uranometria Argentina; we emend to $225=\mathrm{x}$ Vel

Pav 4. Knobel has 32=v Pav; we emend to $33=v$ Pav

\section{A.2. Annotations and emendations to De Houtman}

F 273 and F 274 have $\alpha=330^{\circ} 36^{\prime}$ and $209^{\circ} 45^{\prime}$ in the catalogue, which puts both well outside the constellation. Since the descriptions het hert (the heart) and Een onder dese (one below this) puts F 272 in the body and F 274 below it, we emend to $\alpha=300^{\circ} 36^{\prime}$ and $299^{\circ} 45^{\prime}$. The emended positions are near 6.5,-3.3 in Fig.C.17

\section{A.3. Emendations to Classis}

K 1064 has $\lambda=\varnothing 1100$ in the catalogue, which puts it well outside the constellation. We emend to II 1100 , in accordance with the description Trium supra australe cornu praeced. (the leading of the three above the southern horn).

K 1139 \& K 1140 have zodiacal sign $\uparrow$ for their longitude in Classis, but Kepler notes $\varnothing$ as an alternative. This corresponds to their location in the star catalogue of Ptolemaios, and we make the emendation. Kepler also gives an alternative latitude $\beta=53^{\circ}$ A for K 1139, but since this does not fit its description as being north of K 1140 we do not make this emendation.

\section{A.4. Annotations and emendations to Halley}

E 14 and E 15 have zodiacal sign $M$, in the catalogue; we emend to $\times$.

E 40. The angle to reference star HIP 97649 (lucida Aquilae) is given as the angle to reference star HIP 86032 (caput Ophiuchi) and v.v. in the catalogue. We emend the star names.

E 157 has $\lambda=\bumpeq 3^{\circ} 59^{\prime}$; we emend to $\Omega 8^{\circ} 59^{\prime}$.

E $193=\beta$ Cen has an uncharacteristically large position error $\Delta=44$. 1 . It is used as a reference star for the four stars in Musca: these stars have position errors between 5.2 and 5:9, implying that Halley used a more accurate position for E 193. The table has $\lambda$ of E 193 as MD18 18.5, Halley probably used M19 18.5, which gives $\Delta=5: 9$. We do not emend this, as it may not have been obvious to a contemporary of Halley.

E 300. The angle to reference star HIP 30438 (Canopus) is given as the angle to reference star HIP 52727 (clarior in summ. Roboris Carolini) and v.v. in the catalogue. We emend the star names.

The descriptions of the reference stars given by Halley are listed in Table A.1 together with our identifiation of them, and with the error $\Delta$ in their position.

\section{Appendix B: Notes on individual identifications}

\section{B.1. De Houtman}

F 2, F 3, are near -10.5,4.9 and -9.9,6.5, respectively in Fig.C.1. We identify F 2 with its nearest Hipparcos star, HIP116389 and leave F 3 unidentified; Knobel identifies F 3 with HIP 116389 and F 2 with HIP 116602 near -9.6,1.5. Comparison with Tertia Classis leads us to think that F 3 corresponded to HIP 116602 , but that its position in Houtman is corrupted.

F 7, near $-0.5,-0.1$ in Fig.C.1 is closest to HIP $2383(V=5.7)$ but we identify it with the brighter HIP 2472 below it.

F 14-29, Corona Australis. The pattern of this constellation appears shifted as a whole, and we identify the stars accordingly, which in a number of cases leads us to prefer an Hipparcos counterpart at larger angular distance to the nearest Hipparcos star (Fig.C.2). In all cases, Knobel agrees. For F 19, near 0.5,-3.9 he prefers HIP 93049, which however has $V=6.3$.

F 38, near 12.4,5.3 in Fig.C.4 is identified by Knobel with HIP 13884 , near $13.7,3.7$, brighter but further $\left(V=5.0, d=2^{\circ}\right)$ than our preferred counterpart.

F 42,43 are identified by us with the nearest counterparts HIP 5896 and HIP 4293, respectively, near 2,1.5 in Fig.C.4. Knobel identifies F 42 with HIP 4293 and F 43 with HIP 1647 near $-2.2,1.6$

F 44, near $-0.8,-1.6$ in Fig. C.4, is identfied by Knobel with HIP 865 , a $V=6.7$ star, too faint in our view.

F 46, near 1.8,-1.5 in Fig.C.4 is the Small Magellanic Cloud F 53, F 54, near -7.6,5.2 and -3.8,2.6 in Fig.C.5 are identified by us with HIP 19893 and HIP 21281, respectively. Knobel identifies F 53 with HIP 21281 and F 54 with HIP 23693, near $0.0,1.2$.

F 59, near -3.4,5.2 in Fig.C.6 is identified by Knobel with HIP $26862(V=6.2)$, one magnitude fainter than the counterpart we prefer.

F 77, near -8.2,11.3 in Fig.C.7 is identified by Knobel with HIP 37664, near $-7.4,8.4$, both fainter and further $(V=5.1)$ than our counterpart.

F 78, F 79 near -6.8,13.9 and -5.6,17.9 in Fig.C.7 are identified by Knobel with our counterpart for F 83 and F 82, respectively, near $-5.7,9.0$. and $-5.8,10.7$. 
Table A.1. Reference stars of Halley and their position error.

\begin{tabular}{|c|c|c|c|}
\hline name in Halley & Bayer & HIP & $\Delta\left(^{\prime}\right)$ \\
\hline Spica Virginis & $\alpha$ Vir & 65474 & 2.6 \\
\hline Yed Ophiuchi & $\delta \mathrm{Oph}$ & 79593 & 1.0 \\
\hline lucida colli Serp. & $\alpha \mathrm{Ser}$ & 77070 & 2.7 \\
\hline Lanx Austrina & $\alpha^{2} \mathrm{Lib}$ & 72622 & 3.0 \\
\hline genus Ophiuchi & $\eta \mathrm{Oph}$ & 84012 & 3.7 \\
\hline humerus $(\sin .) \chi^{\top}$ & $\sigma \mathrm{Sgr}$ & 92855 & 7.7 \\
\hline Cor Scorp. & $\alpha \mathrm{Sco}$ & 80763 & 4.2 \\
\hline oculus Pavonis & $\alpha \mathrm{Pav}$ & 100751 & $(6.9)$ \\
\hline lucida Aquilae & $\alpha \mathrm{Aql}$ & 97649 & 2.5 \\
\hline caput Ophiuchi & $\alpha \mathrm{Oph}$ & 86032 & 2.7 \\
\hline sequent. capit. $\nearrow$ & $\pi \mathrm{Sgr}$ & 94141 & 2.5 \\
\hline 5tus spond. caud. & $\theta$ Sco & 86228 & $(5.8)$ \\
\hline lucida Arietis & $\alpha$ Ari & 9884 & 0.8 \\
\hline Aldebaran & $\alpha$ Tau & 21421 & 1.3 \\
\hline (Lux) Mandibula Ceti & $\alpha$ Cet & 14135 & 2.1 \\
\hline Aust. Cadae Ceti & $\beta$ Cet & 3419 & 2.0 \\
\hline Regel Orionis & $\beta$ Ori & 24436 & 1.8 \\
\hline Achernar & $\alpha$ Eri & 7588 & (7.6) \\
\hline Fomalhaut & $\alpha$ PsA & 113368 & 5.9 \\
\hline Cor Hydrae & $\alpha$ Нуа & 46390 & 1.6 \\
\hline Sirius & $\alpha \mathrm{CMa}$ & 32349 & 0.9 \\
\hline Marchab Pegasi & $\alpha$ Peg & 113963 & 1.3 \\
\hline Procyon & $\alpha \mathrm{CMi}$ & 37279 & 1.5 \\
\hline sect.trans.(Navis) & $\lambda \mathrm{Vel}$ & 44816 & (3.1) \\
\hline Canobus & $\alpha$ Car & 30438 & (3.5) \\
\hline Lucida in Tran. & $\zeta$ Pup & 39429 & $(3.2)$ \\
\hline Cauda Leonis & $\beta$ Leo & 57632 & 2.0 \\
\hline Cor Leonis & $\alpha$ Leo & 49669 & 0.6 \\
\hline Lanx Borea & $\beta \mathrm{Lib}$ & 74785 & 2.5 \\
\hline pes (dext.) Cent. & $\alpha$ Cen & 71683 & $(4.7)$ \\
\hline Pes Crucis & $\alpha^{1} \mathrm{Cru}$ & 60718 & $(5.8)$ \\
\hline Aust.(Part.)arc. $\nearrow^{\nearrow}$ & $\epsilon \mathrm{Sgr}$ & 90185 & (5.4) \\
\hline Caput Phoenicis & $\alpha$ Phe & 2081 & (5.6) \\
\hline lucida ad rad. Roboris Carolini & $\beta$ Car & 45238 & $(5.2)$ \\
\hline genus sinistr. Cent. & $\beta$ Cen & 68702 & $(44.1)^{a}$ \\
\hline clarior.in summ. Roboris Carolini & $\mu \mathrm{Vel}$ & 52727 & $(3.2)$ \\
\hline ult.Erid.Ptol. & $\theta^{1}$ Eri & 13847 & $(6.8)$ \\
\hline ala gruis & $\alpha \mathrm{Gru}$ & 109268 & $(5.8)$ \\
\hline
\end{tabular}

Notes. The position errors are taken from KeplerE, those in brackets refer to stars not in KeplerE, and are taken from Halley. Small variations in the descriptions given by Halley are ignored; words between brackets are occasionally omitted by him.

${ }^{a}$ see annotation for E 193 in Sect.A.4

F 82, F 83, near -3.8,10.5 and -3.6,9.1 in Fig.C.7 are identified by Knobel with HIP 40091 and HIP 40326, near -2.1,10.4 and $-1.6,9.7$, respectively.

F 115, F 116, near 18.9,3.0 and 18.7,1.5 in Fig.C.7 F 115 is unidentified by Knobel, and F 116 with our counterpart for F 115 .

F 117, F118, the close pair near 20.5,-0.8 in Fig.C.7 are identified by Knobel with the pair HIP 51561, HIP 51610 near 22.1,1.3.

$\mathrm{F} 119, \mathrm{~F} 120, \mathrm{~F} 121$ three stars near near $22.1,-1.9$ to -4.8 in Fig.C.7 are identified by Knobel with our counterpart for F 117 near 20.8,-0.8, and F 119, F 120, respectively, i.e shifted by one counterpart upwards.

F 142-F 146, five stars near 0,0 in Fig. C.8 form a pattern with four stars in a vertical row and one star to the left. We follow Knobel in his identification which implies a shift to the south, taking further stars as identifications for four stars. The alternative would leave the top star, F 145, unidentified.
F 195-197, near -9,3 in Fig.C.12, are closely matched with our counterparts, and less so by those proposed by Knobel. He identifies F 195 with HIP 69671, too faint at $V=6.3$; F 195 with HIP 70054 at $-9.6,5.4$, more than $2^{\circ}$ distant, and F 196 with our counterpart for F 195 .

F 199, near -7.8,3.5 in Fig.C.12 is closest to HIP 70915 ( $V=5.5, d=16$ ' 7 , but we consider the brighter HIP 70576 the more likely, albeit further counterpart.

F 203, near $-2.0,-3.5$ in Fig.C.12 is identified by Knobel with HIP 73345 ( $\left.V=6.8, d=55^{\prime} 9\right)$, much fainter and only marginally closer than our preferred counterpart.

F 214, near 0.9,0.3 in Fig.C.12 is closest to HIP 75181 ( $V=5.7$, $d=33$.4), but we slightly prefer the somewhat brighter but further HIP 75206.

F 228-231, from $-5,-6$ to $-6,-1$ in Fig. C.14 We shift these stars south by about 1.5 , after which each is identified with the then nearest star, thereby identifying these four stars with the four brightest nearby Hipparcos stars.

F 232-233, near -2.5,-0.5 in Fig.C.14 are both closest to HIP 80047/80057. (These Hipparcos stars are separated by $1: 7$ and cannot be separated by the naked eye.) We identify F 232 with the Hipparcos pair, and F 233, to the south of F 232, with the star east and south of the Hipparcos pair, viz. HIP 81065.

F 236, near 9.3,1.6 in Fig.C.14 is identified by Knobel with HIP 92394, much fainter and somewhat further $(V=6.0, d=64.5)$ than our preferred counterpart.

F 249, near $-5.4,2.5$ in Fig.C.16 is closest to HIP 82545 $(\mu$ Sco, $V=3.6, d=7.7)$, but we take the brighter HIP 82514 $\left(\mu^{1} \mathrm{Sco}\right)$ as more plausible counterpart. The two Hipparcos stars are separated by 5:8 only.

F 250, F 251, near $-4.7,-1.4$ and $-4.5,-1.9$ in Fig.C.16. Knobel identifies these with the close (7.5) pair HIP 82671, HIP 82729 ( $\zeta^{1}$ and $\zeta^{2}$ Sco); we identify F 250 with the nearer, but fainter Hipparcos star.

F 258 is almost equidistant from HIP 86929 and HIP 88866. Because HIP 86929 is already identified with F 257, we identify F 258 with HIP 88866

F 257-F 275, i.e. the whole constellation Pavo (Fig.C.17), form a pattern which appears to match the Hipparcos stars best after a shift of about 1.5 to the north-east. This explains several differences in our identifications with those by Knobel. Our emendations to F 273 and F 274, where Knobel has no and a different identification, respectively, explain our different identifications for these stars. F 268, near 2.6,-2.4, is close to the pair HIP 98478 - HIP 98624 (which is separated by 8.7), we choose the brighter of the two as counterpart.

F 304, near 4.0,-0.7 in Fig.C.20 is the counterpart of the close $\left(d=300^{\prime} 0\right.$, i.e. inseparable by the naked eye) pair HIP 2484/HIP 2487 ( $\beta$ Tuc).

\section{B.2. Secunda Classis and Tertia Classis}

In this section, we abbreviate reference to Figures C.n in Paper I with PI-C.n.

K 1006, near 10.6,3.6 in Fig. PI-C.7, is closest to HIP 74596, but that star is already matched with K 142; we identify K 1006 with the next nearest star, near 12.1,4.2.

$\mathrm{K} 1009$, near $-1.4,3.2$ in Fig. PI-C.9 is M 13

$\mathrm{K} 1010$, near 8.9,14.9 in Fig. PI-C.11, is closest to HIP 101243 ( $\omega^{2}$ Cyg, $V=5.4, d=67$ '9), but we choose HIP $101138\left(\omega^{1}\right.$ Cyg $)$ as a brighter, slightly further counterpart. The angle between HIP 101138 and HIP 101243 is 20'3, and perhaps K 1010 - indicated nebulous in the catalogue, is the combined light of these two stars. 
$\mathrm{K} 1011$, near 4.8,-14.4 in Fig. PI-C.15, is nearest to HIP 19335 $(V=5.5, d=68$.7) but we identify it with the further but brighter HIP 19811, near 6.1,-13.3.

K 1012-K 1042: Ophiuchus and Serpens. Ophiuchus in particular has many bad and/or uncertain position in KeplerE. Many stars in Secunda Classis are repeats of stars in KeplerE, sometimes with rather better positions (see Table 10.

$\mathrm{K} 1036$ is the supernova of 1604 . For the modern position we use $\alpha(2000)=17 \mathrm{~h} 30 \mathrm{~m} 36 \mathrm{~s}, \delta(2000)=-21^{\circ} 28^{\prime} 56^{\prime \prime}$.

K 1054, near 4.5,7.3 in Fig. PI-C.30, repeat entry for K 504

K 1055, near 16.6,11.7 in Fig. PI-C.45, repeat entry for K 823

K 1072, near $-10.5,5.8$ in Fig. PI-C.31, repeat entry for K 323

$\mathrm{K} 1084$, near $-6.3,2.9$ in Fig.C.16 is close to the pair HIP 82514/HIP 82545 (separation 5.8) and perhaps represents their combined light

K 1095, near 5.7,14.8 in Fig.C.16 Kepler mentions a latitude aliter (alternatively) for this star, 430 A, three degrees further south, which would lead to identification with HIP 87072, near 6.5,11.9.

K 1099, near -1.8,11.8 in Fig. C.24 is described In oculo nebulosa duplex (a double nebulous [star] in the eye), corresponding to the close (13'.9) pair HIP9ं2761/92845 $=v_{1} / v_{2}$ Sgr.

$\mathrm{K} 1104$ and K 1105 , near $0.7,-11.8$ and $0.1,-6.9$ in Fig. C.24 are identified by us with the two bright stars further East, $\beta$ and $\alpha$ Sgr, near 2.0,-10.6 and 2.8,-6.9, respectively.

K 1147, near 11.5,17.0 in Fig.C.6, lies almost exactly between two stars of different brightness, HIP 33077 to the North and HIP 33092 to the South; we take the brighter star as the counterpart.

K 1148 repeat of K 938

K 1152, near 12.7,32.3 in Fig.C.6. We identify this star with HIP 33184, northwest of it, but perhaps HIP 33971, to the southeast, is also possible, being brighter but further $(V=5.0$, $\left.d=135.5^{\prime}\right)$.

$\mathrm{K} 1170$, near $-12.1,16.7$ in Fig. C.7 is surrounded by faint $(V<5.0$ stars, but we choose a brighter star near $-11.2,17.4$ as counterpart.

K 1171, K 1173 and K 1174, respectively near -12.4,11.2, $-8.3,10.8$ and $-7.5,12.1$ in Fig. C.7 are identical to the brightest stars from the Hipparcos Catalogue near each of them, even though fainter stars are closer.

$\mathrm{K} 1178$, close to the much brighter K 1176, both near -3.9,9.5 in Fig.C.7is the faint star close to the bright counterpart of K 1176 , $\zeta$ Pup.

K 1230, near $-2.4,2.0$ in Fig. C.8 is the globular cluster $\omega$ Cen. We use the magnitude and position given by Harris (1996, version of February 2003).

K 1237-K 1243, K 1246. These southern stars in Centaurus are badly matched. Comparing their pattern with the bright stars from the Hipparcos Catalogue we suggest that they are located about $4^{\circ}$ too far south and varying degrees too far west in Secunda Classis, and we have chosen counterparts accordingly. See also Fig.2,

K 1244, near -2.7,-1.8 in Fig.C.8 has no obvious counterpart. Even though HIP 61932 is very close (3') to K 1232, we prefer to identify K 1244 with it and K 1232 with HIP 61622 just above it. An alternative is to emend the zodiacal sign of its longitude from

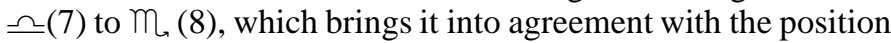
in the catalogue of Ptolemaios and leads to identification with HIP 71683 ( $\alpha$ Cen).

$\mathrm{K} 1245$, near 4.5,-11.6 in Fig.C.8, is identified with HIP 68702 $(\beta$ Cen $)$.

K 1266-K 1272, Ara (Fig.C.15). There is a general shift of the catalogue positions of this constellation to the East, leading to many cases where the obvious and correct Hipparcos counterpart is not the nearest Hipparrcos star.

K 1273-1285, Corona Australis (Fig.C.2). There appears to be a general shift in the coordinates of the stars in this constellation, leading us to accept Hipparcos counterparts at larger angles in many cases where nearer Hipparcos stars are present.

K 1296, near -1.4,-3.6 in Fig.C.27, has the same counterpart as a star from Grus in Tertia Classis, K 1303.

$\mathrm{K} 1300$, near -9.0,-2.5 in Fig.C.27, is closest to HIP 104738, which however is already taken by K 1298, hence we suggest a faint star to the North as counterpart, HIP 104752 near $-8.3,-0.7$.

K 1302, near -7.5,3.5 in Fig.C.27 is tentatively identified not with the nearest star to the west, but with the brighter one beyond it, near $-9.6,4.0$.

K 1408 in Fig.C.5 is the Large Magellanic Cloud

K 1433, near 2.4,-3.1 in Fig.C.4 is the Small Magellanic Cloud

\section{B.3. Halley}

E 1, near $-10.4,10.1$ in Fig. D.1, is the combined light of the close (15") pair HIP 78820/HIP78821 ( $\left.\beta^{1} \mathrm{Sco} / \beta^{2} \mathrm{Sco}\right)$.

E 20, near 3.1,-9.8 in Fig.D.1 is near the open cluster NGC 6231, which is probably why Halley calls it nebulous.

E 21, near 3.3,-10.3 in Fig. D.1 is closer to HIP 82671 ( $V=4.7$, $d=4.0^{\prime}$ ) than to the brighter counterpart we prefer.

E 29, near 14.6,-2.6 in Fig.D.1. The Hipparcos object that we identify this with is a member of the open cluster M 7 .

E 146, near -1.1,2.7 in Fig.D.8, is $\eta$ Car. This highly variable star is not in the Hipparcos catalogue; it is HR 4210. Halley's catalogue is the first known reference to this star.

E 180, near $-4.3,0.3$ in Fig. D.10, is the globular cluster $\omega$ Centauri.

E 212-E 217. Halley notes that these last five stars of Lupus, in the upper left corner of Fig.D.11, were observed while sailing (inter navigandum), and thus less accurate but sufficiently accurate for use on a globe. The Figure shows that these positions are indeed less accurate than the others.

E 282, near 1.1,-2.7 in Fig. D.18, corresponds to the close (1.7') pair HIP 80047/HIP80057 ( $\delta^{1} / \delta^{2}$ Aps $)$

E 311, near 1.1,-7.4 in Fig. D.21, corresponds to the combined light of the close (13") pair HIP 34473/HIP 34481.

E 314, near 1.3,-1.6 in Fig.D.21, corresponds to the combined light of the close (1'7) pair HIP 40817/HIP 40834. 


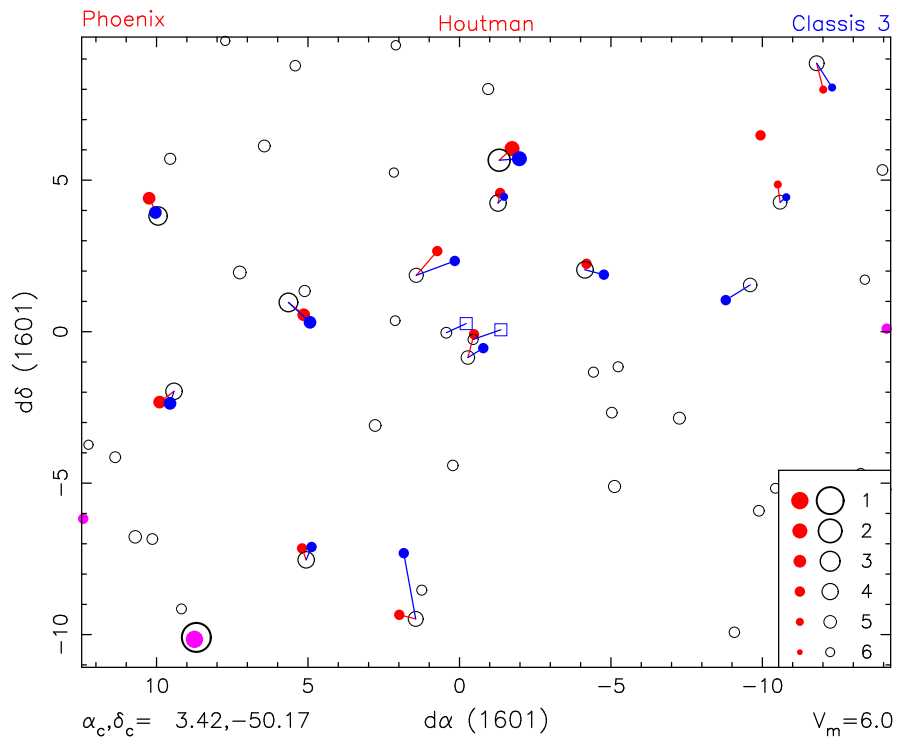

Fig. C.1. Phoenix

\section{Appendix C: Constellations in Houtman and Classis}

To illustrate and clarify our identifications we provide figures for each constellation, starting with the constellations in Houtman. To minimize deformation we rotate the approximate center of the constellation to point $\Upsilon$, as explained in Appendix C of Paper I, but now for equatorial rather than ecliptic coordinates. The values used for this center $\alpha_{\mathrm{c}}, \delta_{\mathrm{c}}$ are indicated with each Figure, as is the magnitude limit to which we also show all Hipparcos stars $V_{\mathrm{m}}$ (usually $V_{\mathrm{m}}=6.0$ ). The magnitudes of both catalogue and Hipparcos stars are indicated with symbol size.

In illustrating the entries from Secunda Classis and Tertia Classis we wish to facilitate comparison with the catalogues KeplerE on one hand, and Houtman on the other hand. When Secunda Classis only adds a small number of stars to a constellation in KeplerE, we have shown these added stars in yellow in the figures for KeplerE in Paper I, as indicated in Table2 When the constellation in Secunda Classis shows a large overlap with a constellation in Houtman, and for all constellations in Tertia Classis, we show the stars from Secunda Classis or Tertia Classis in the figure for the corresponding constellation in Houtman, as indicated in Tables 2 and 3 . To do so we convert the ecliptic positions as given in Secunda Classis and Tertia Classis to equatorial positions, using the modern value for the obliquity in 1601, $\epsilon=23^{\circ} .491$. (Kepler and Brahe used $\epsilon=23^{\circ} .525$.) Constellations in Secunda Classis which show little or no overlap with Houtman are shown together with stars from KeplerE, in rotated ecliptic coordinates.

In these figures the stars plotted from the first catalogue are shown in red when member of the illustrated constellation, and purple when not a member. Stars from a second catalogue in the same figure and member of the constellation are shown in blue, and other stars in light-blue.

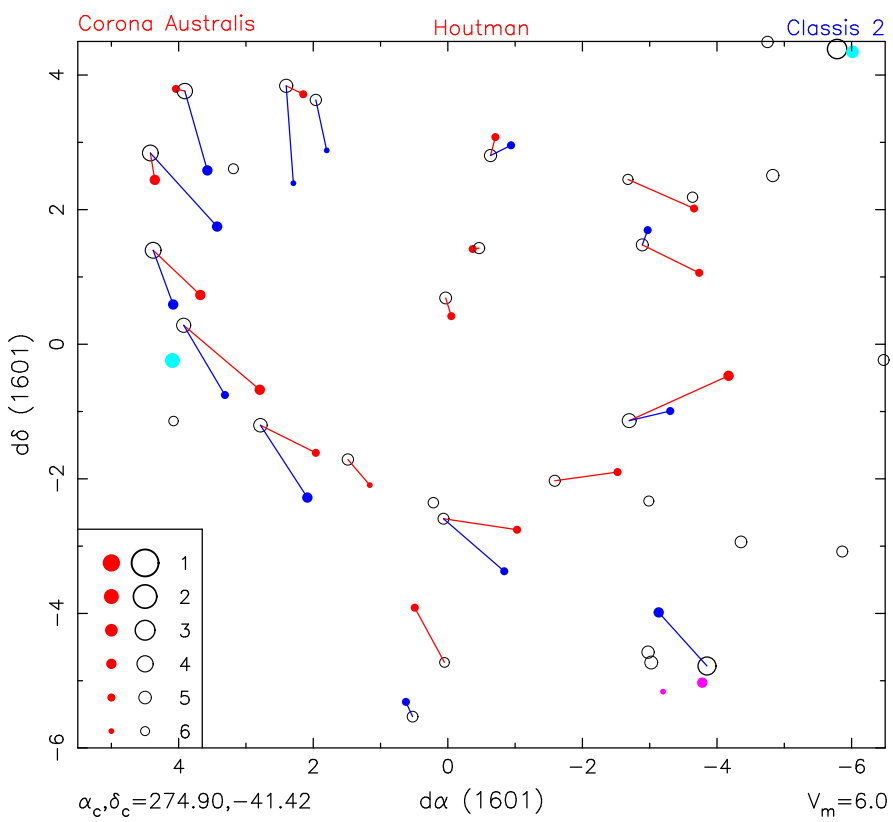

Fig. C.2. Corona Australis

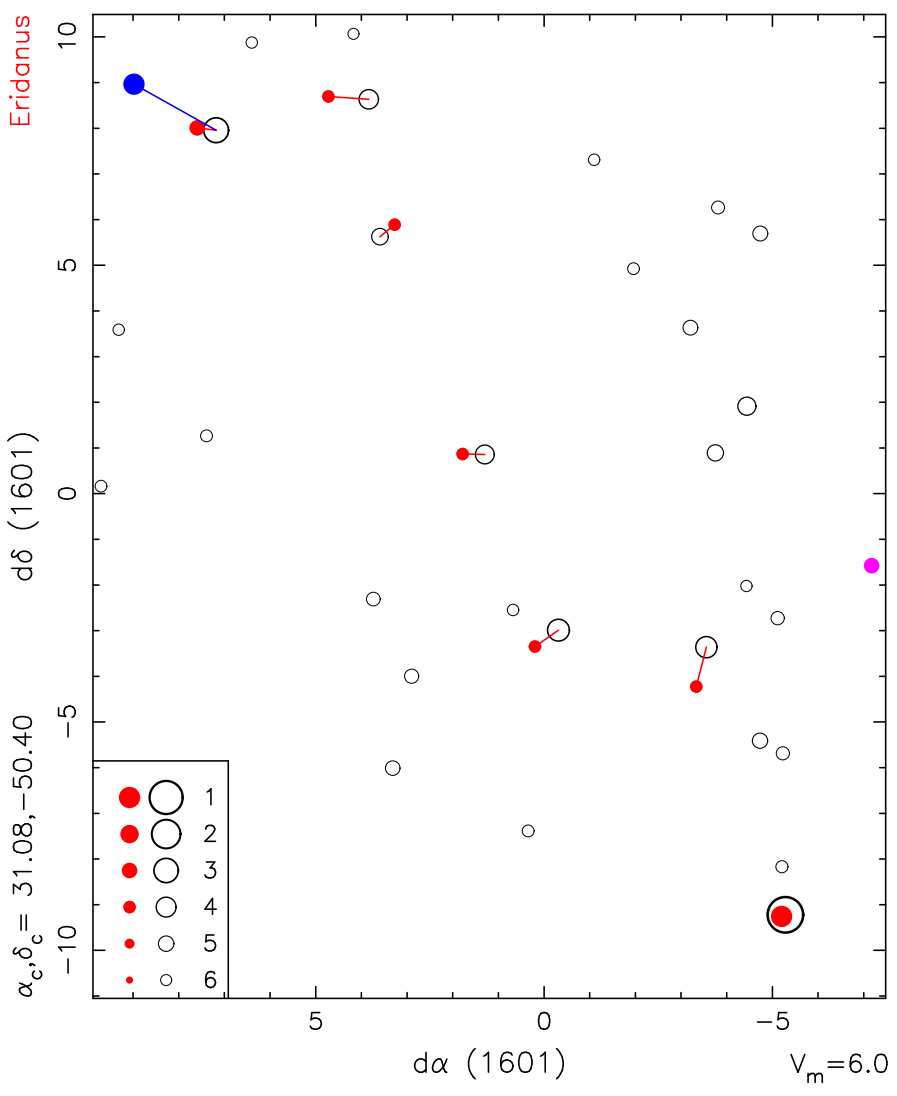

Fig. C.3. Eridanus in Houtman. K 1146, the only star in Classis in common with Houtman, is indicated in blue. See Fig.C.25, where it is at $-10.6,-16.0$. 
Frank Verbunt and Robert H. van Gent: Early star catalogues of the southern sky

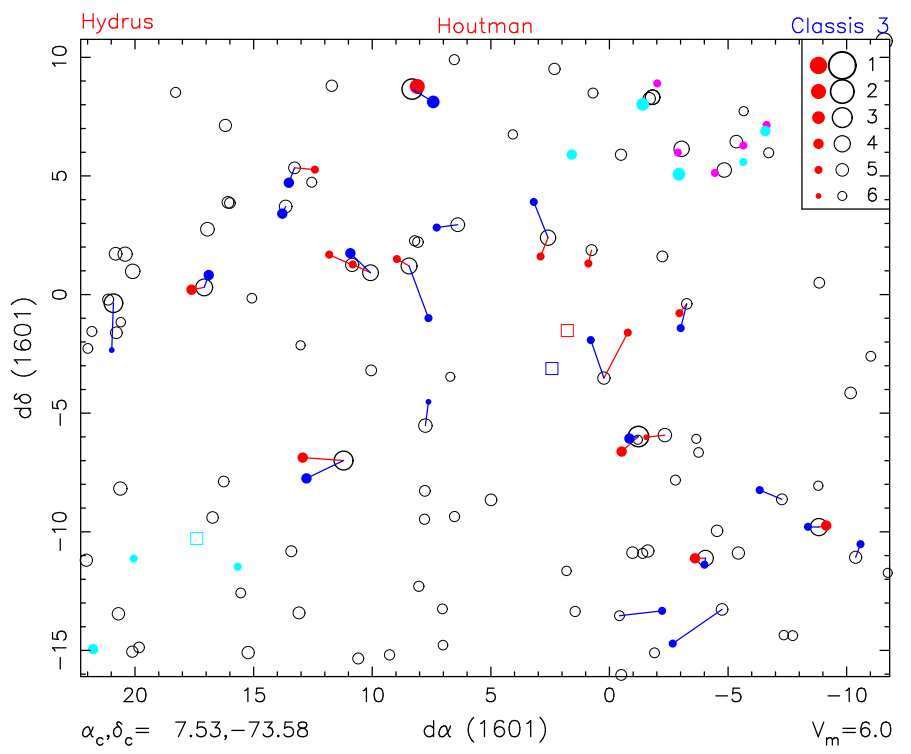

Fig. C.4. Hydrus, with the Small Magellanic Cloud. The nebulous object near 1,-3 is the Small Magellanic Cloud, present both in Houtman and Tertia Classis.

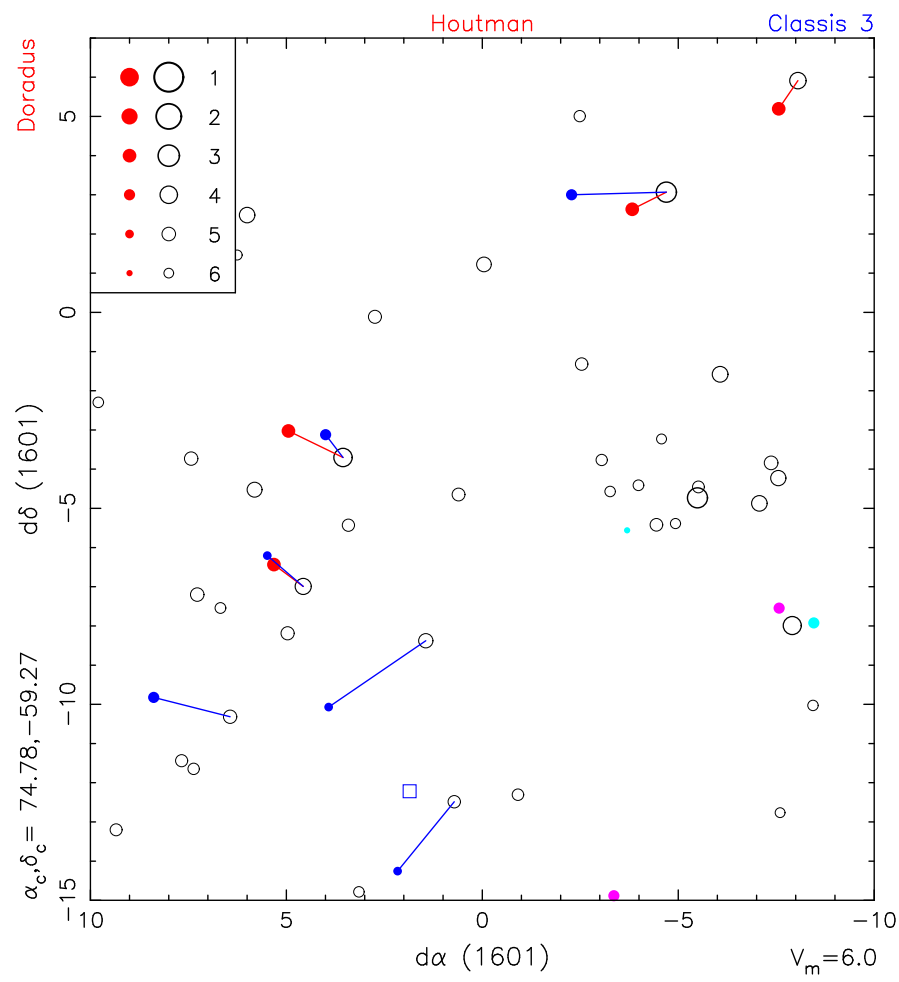

Fig. C.5. Doradus. The nebulous object near 2,-12.5 is the Large Magellanic Cloud, present in Tertia Classis, but not in Houtman.

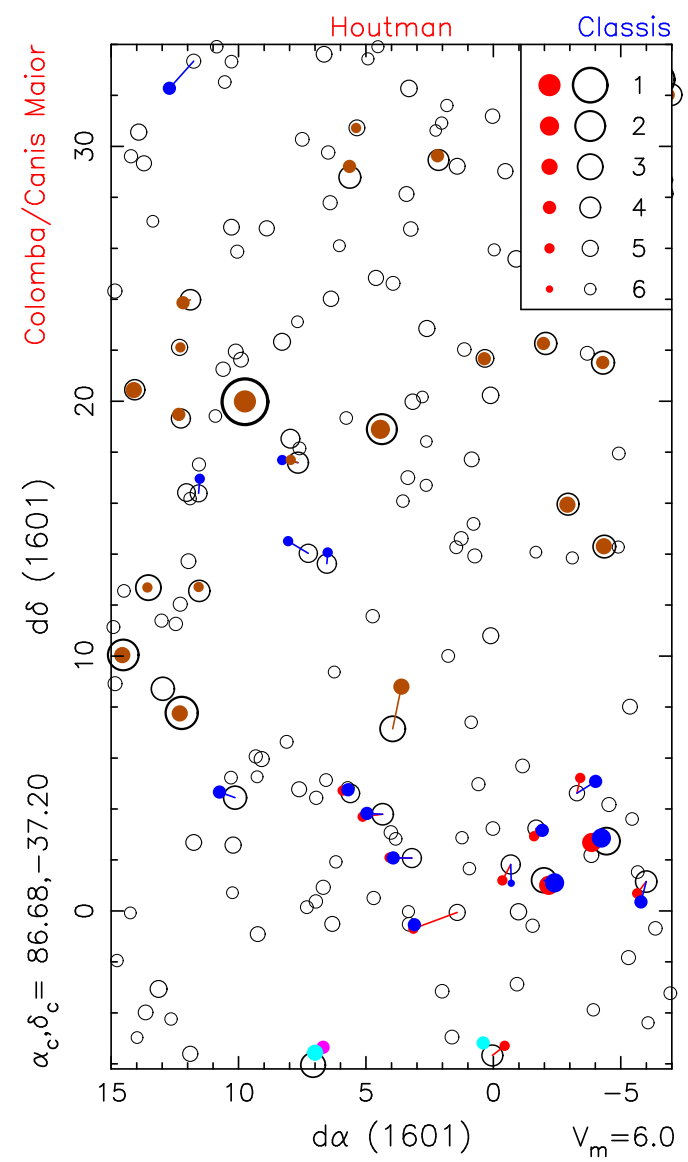

Fig. C.6. Colomba in Houtman is part of Canis Maior in Secunda Classis. Stars in KeplerE are indicated in brown.

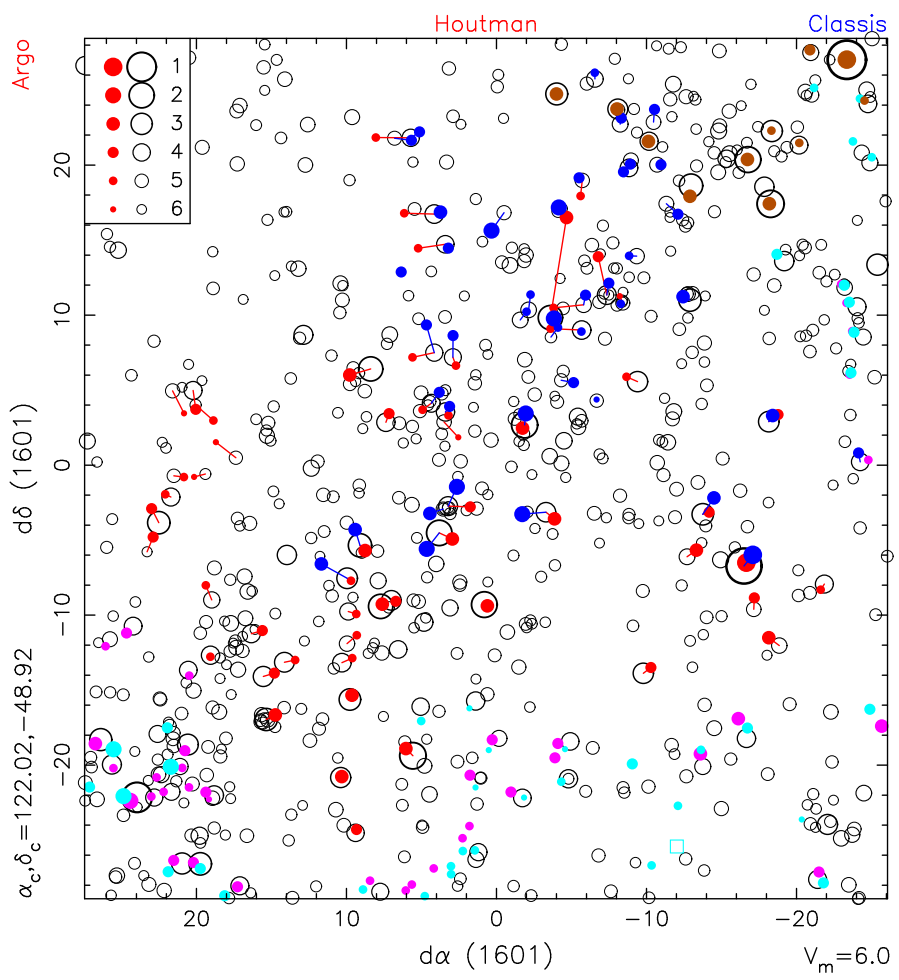

Fig. C.7. Argo. The crowded region near $-6,10$ is hard to interpret. We have chosen to identify the brightest stars in Houtman with the brightest stars in that area, even if this leads to large positional shifts. Stars in KeplerE are indicated in brown. 
Frank Verbunt and Robert H. van Gent: Early star catalogues of the southern sky

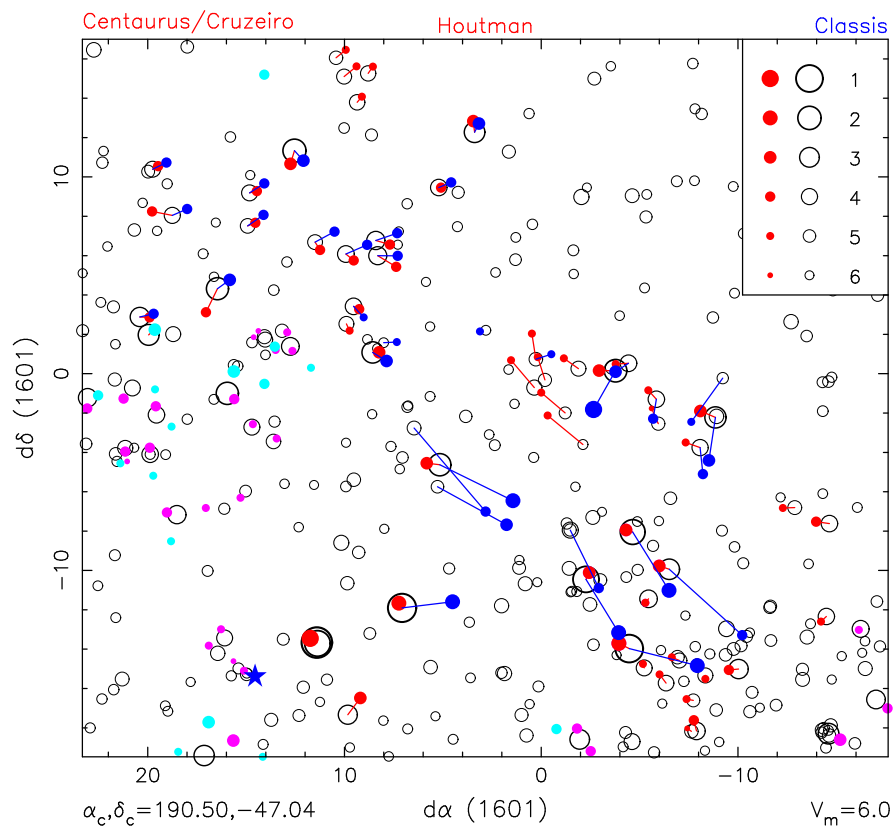

Fig. C.8. Centaurus and Crux. The bright star K 1244, near $-2.7,-1.8$ is tentatively identified by us with HIP 61932 ( $\gamma$ Cen); an alternative position is indicated with a blue star, and leads to identfication with HIP 71683 ( $\alpha$ Cen).

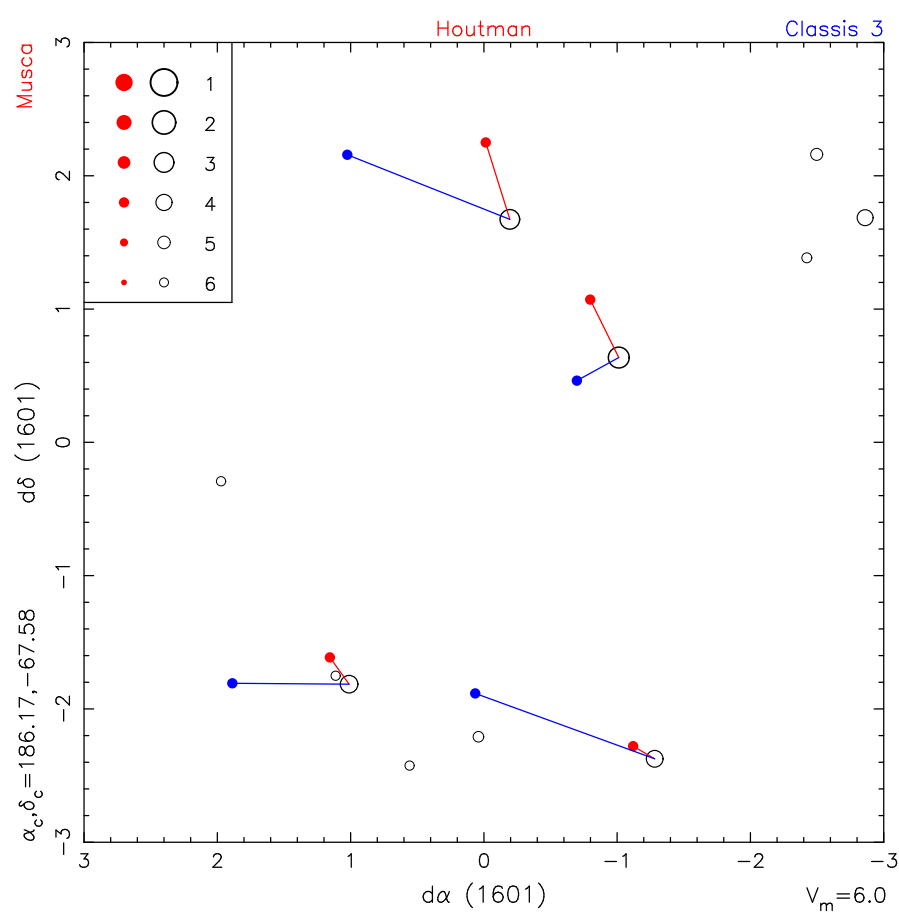

Fig. C.9. Musca

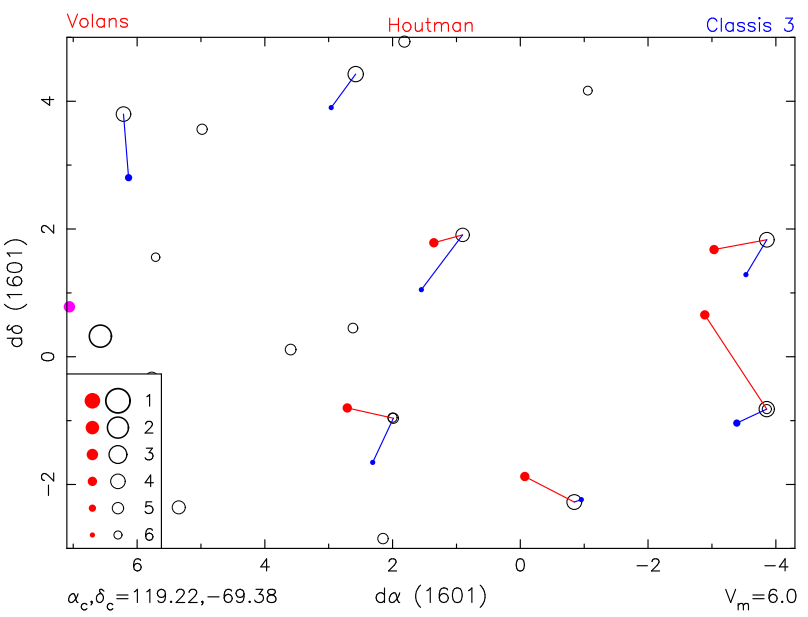

Fig. C.10. Volans

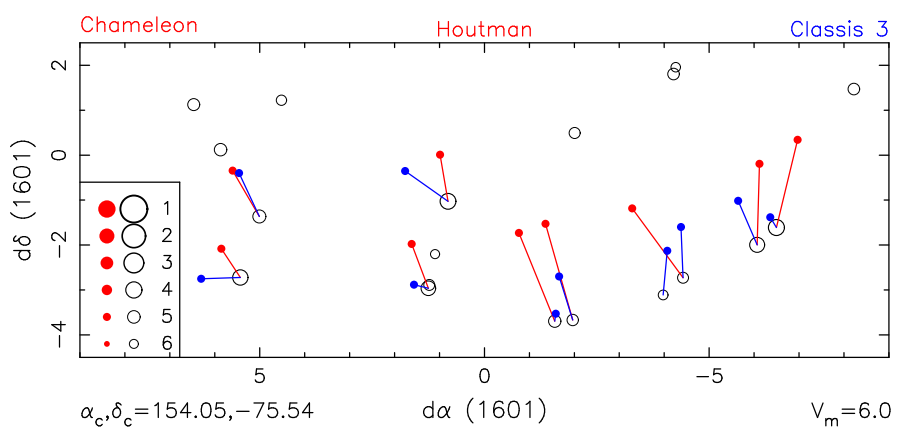

Fig. C.11. Chamaeleon

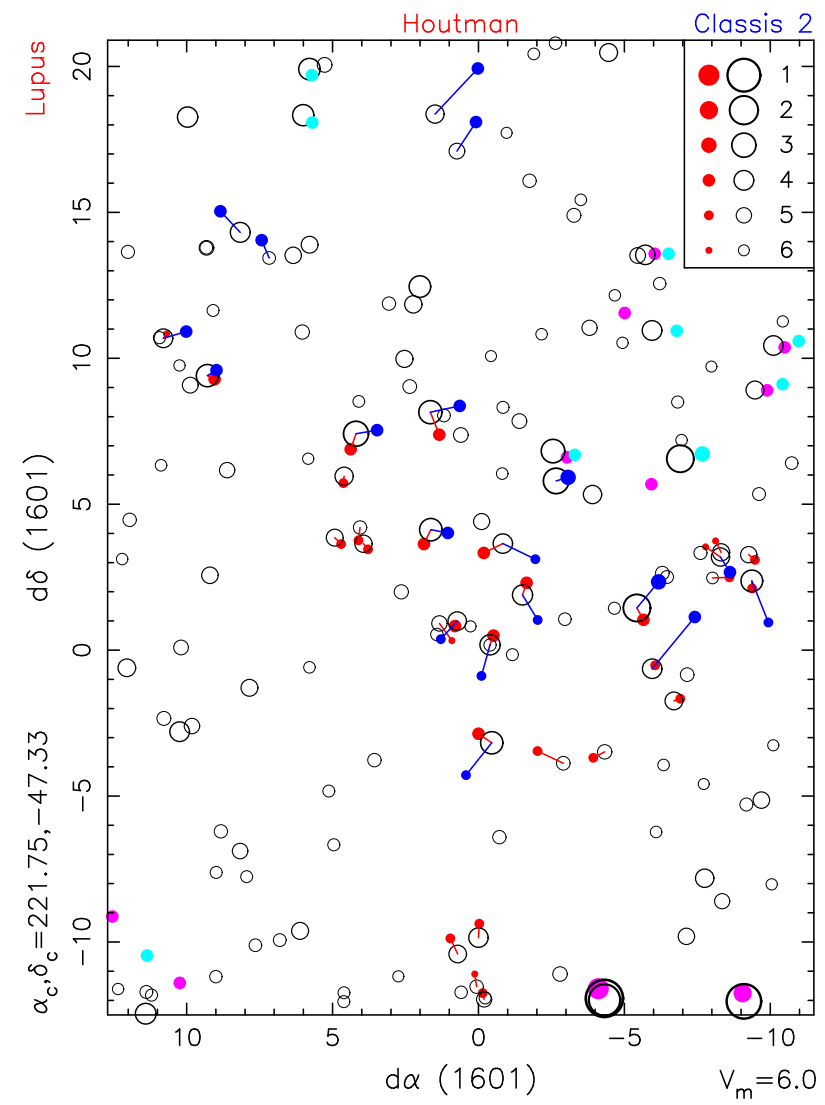

Fig. C.12. Lupus 
Frank Verbunt and Robert H. van Gent: Early star catalogues of the southern sky

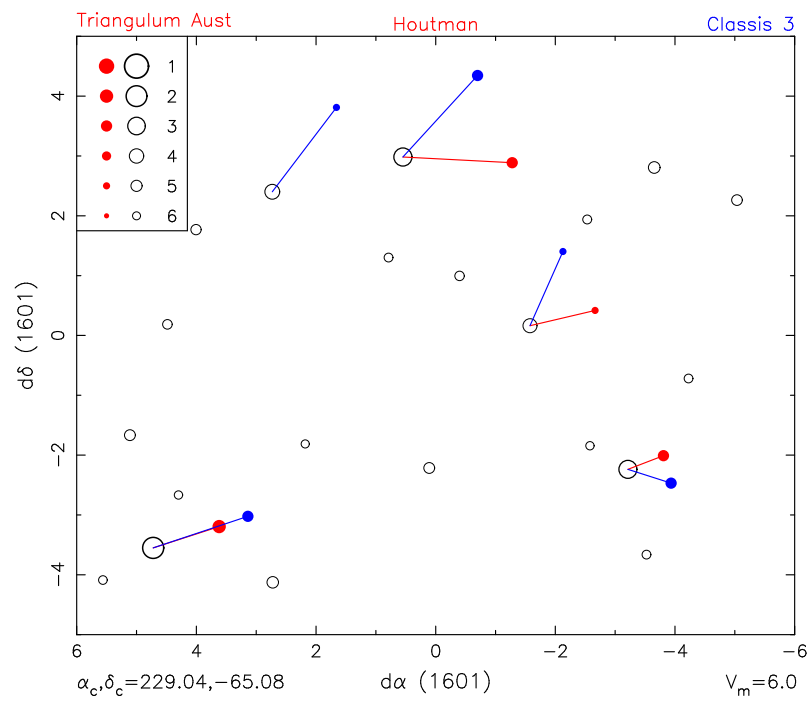

Fig. C.13. Triangulum Australe

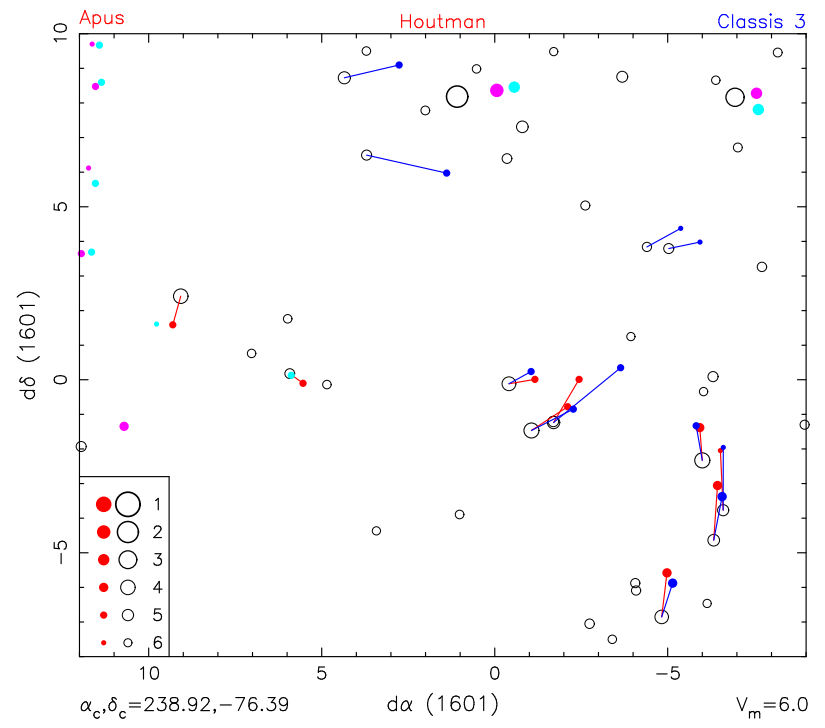

Fig. C.14. Apus

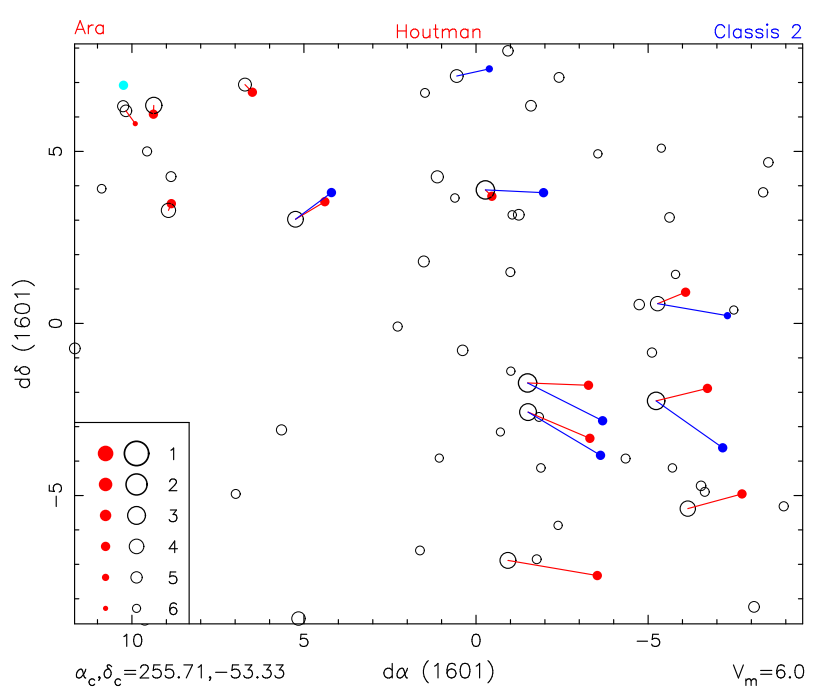

Fig. C.15. Ara

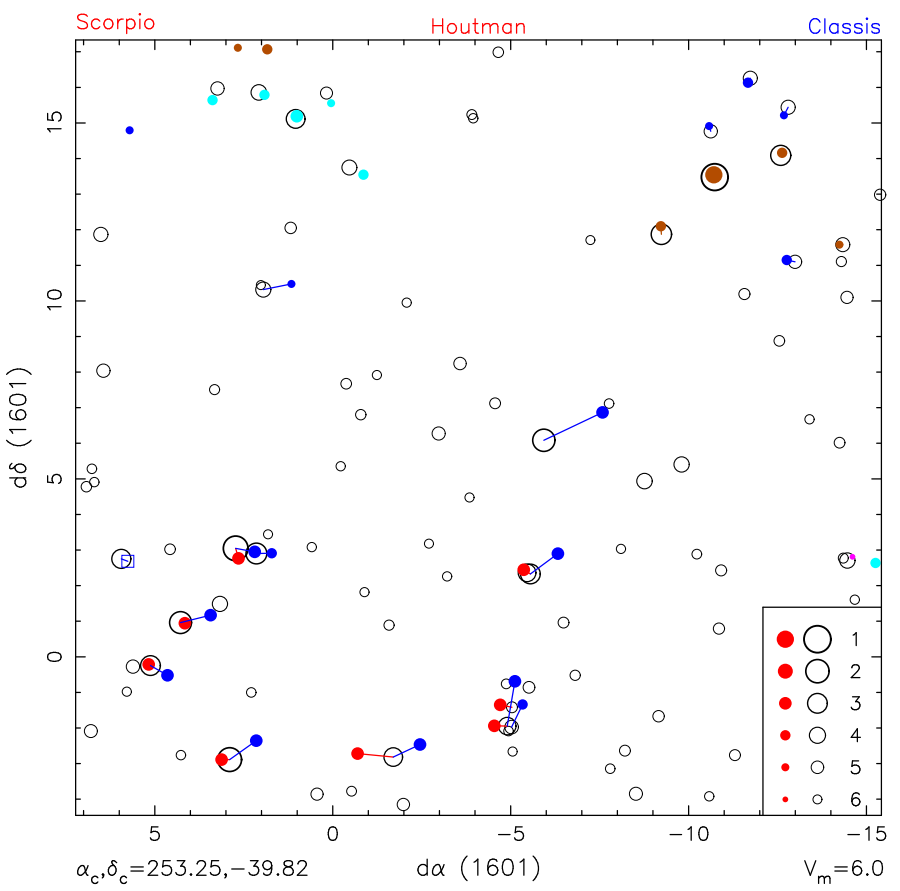

Fig. C.16. Scorpius. Stars in KeplerE are indicated in brown.

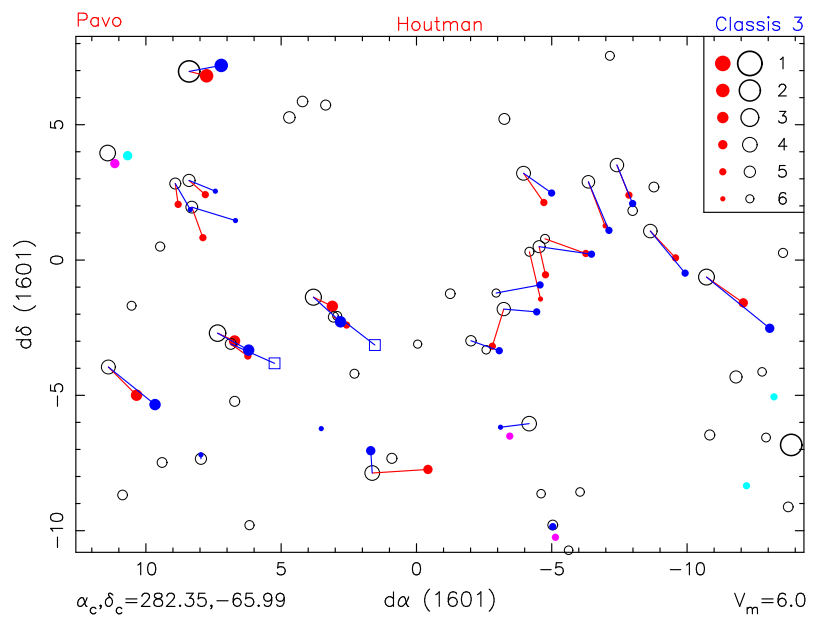

Fig. C.17. Pavo

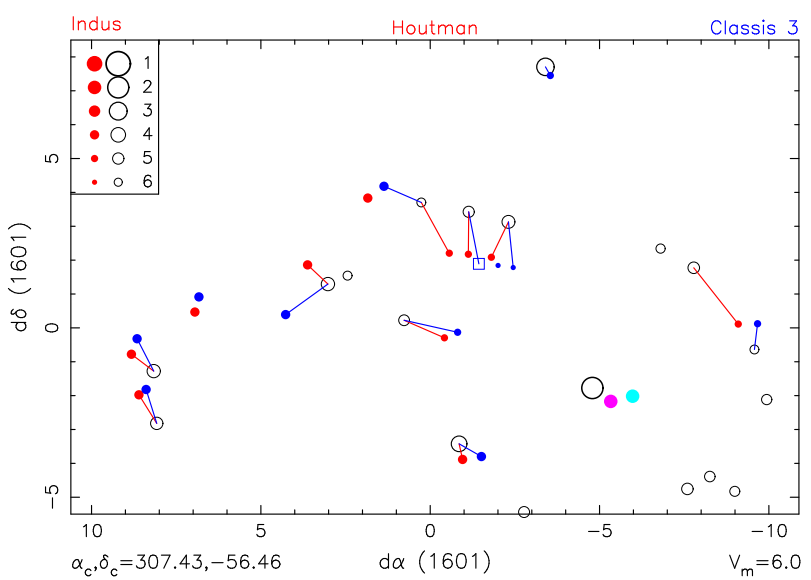

Fig. C.18. Indus. See also Fig.1, 
Frank Verbunt and Robert H. van Gent: Early star catalogues of the southern sky
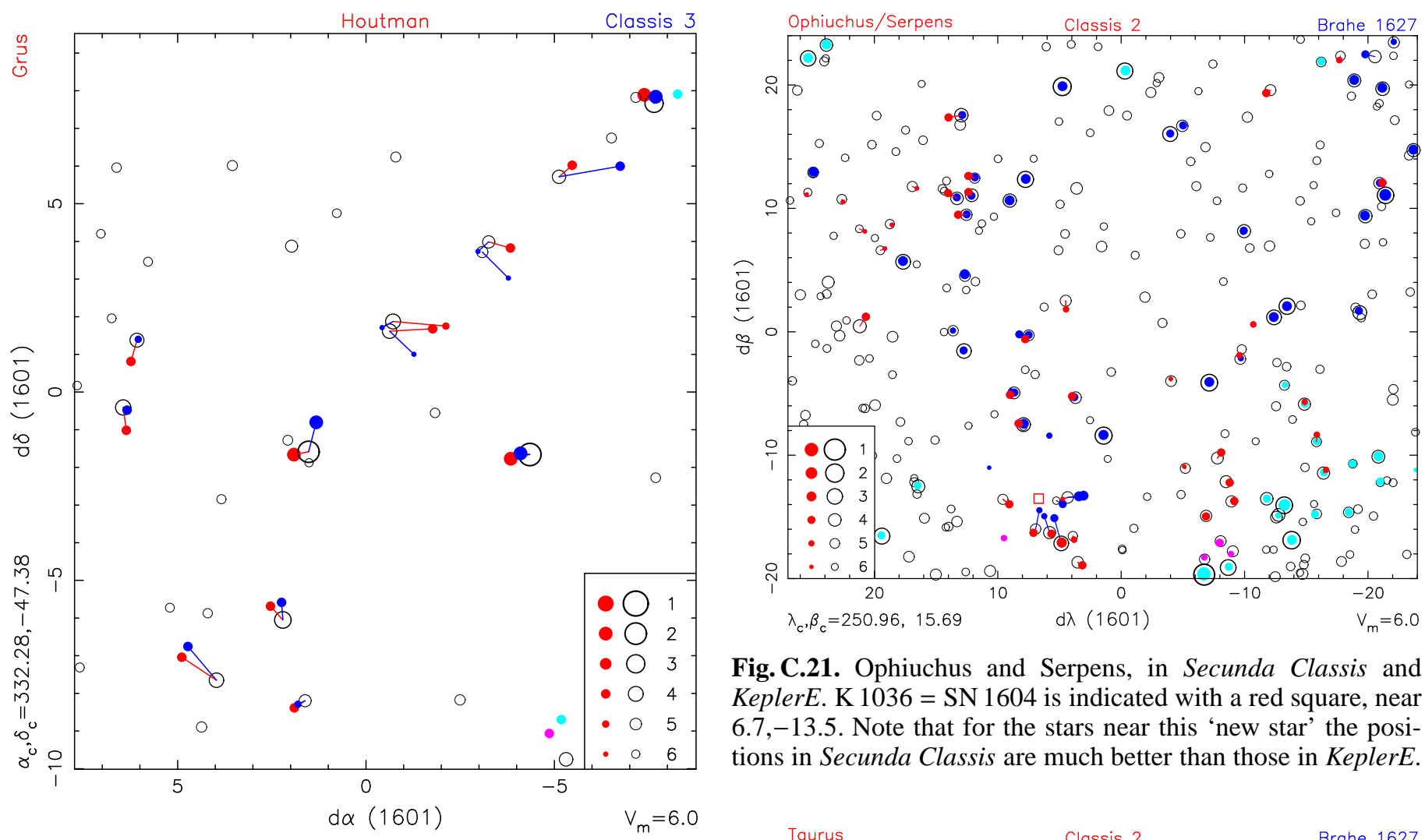

Fig. C.19. Grus

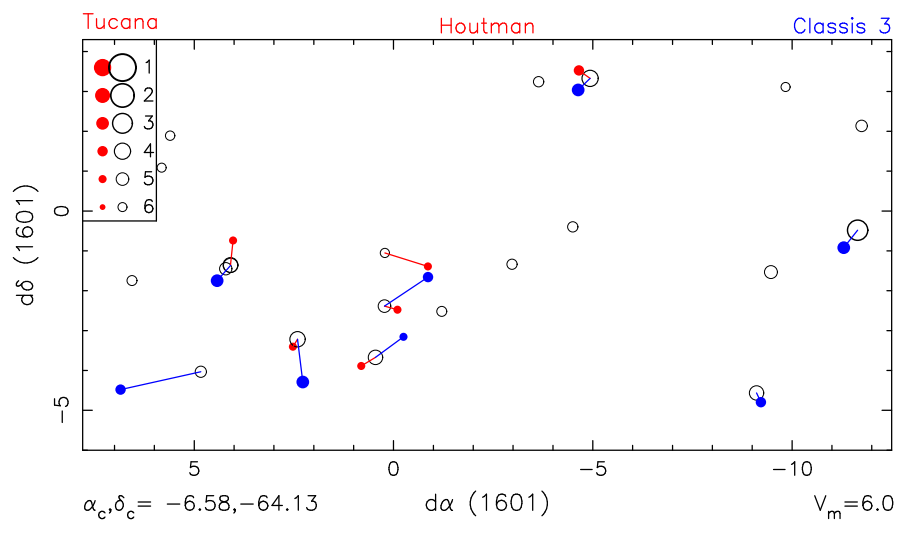

Fig. C.20. Tucana

Fig. C.21. Ophiuchus and Serpens, in Secunda Classis and KeplerE. K 1036 = SN 1604 is indicated with a red square, near 6.7,-13.5. Note that for the stars near this 'new star' the positions in Secunda Classis are much better than those in KeplerE.

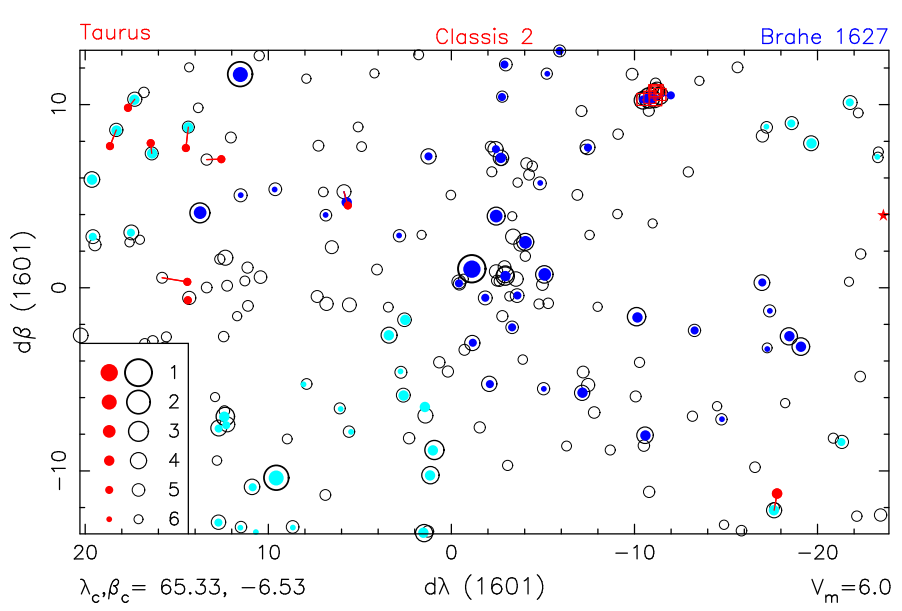

Fig. C.22. Taurus, in Secunda Classis and in KeplerE. The red star at the right gives the position of K 1064 before emendation. For Pleiades, see Fig.C.23. 
Frank Verbunt and Robert H. van Gent: Early star catalogues of the southern sky

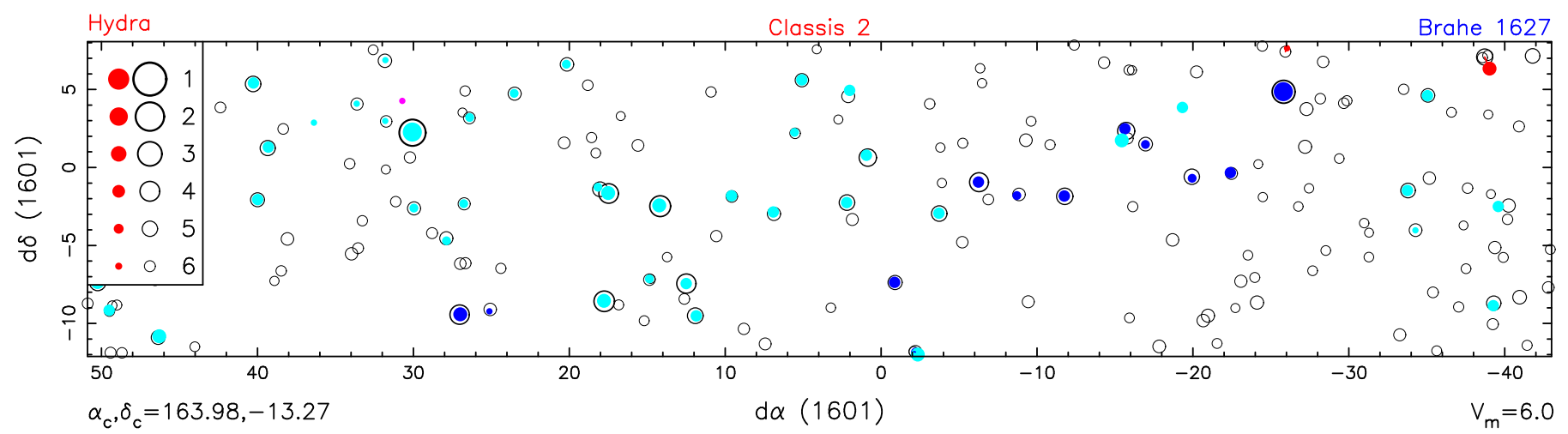

Fig. C.26. Hydra.

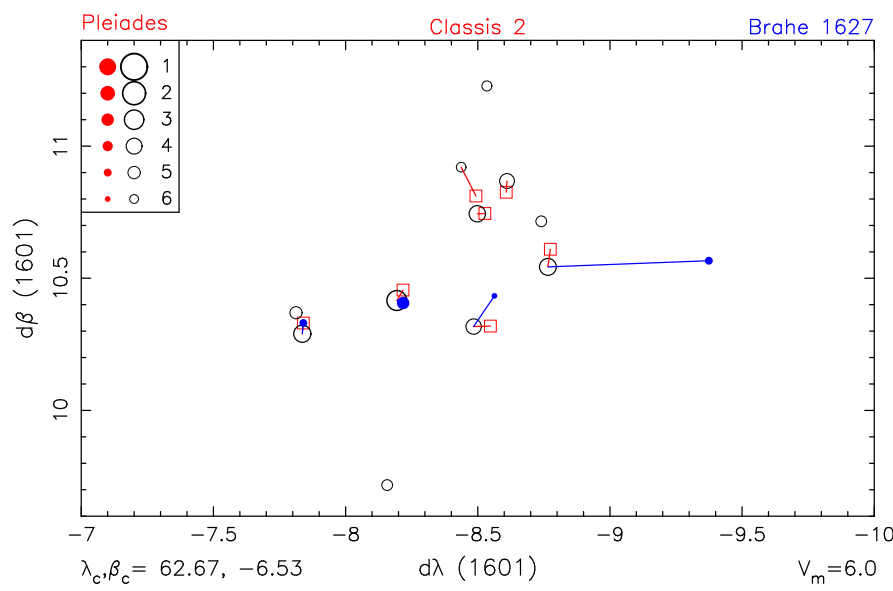

Fig. C.23. Pleiades. Stars from the Pleiades in Secunda Classis are plotted as squares since Secunda Classis does not give magnitudes for these stars.

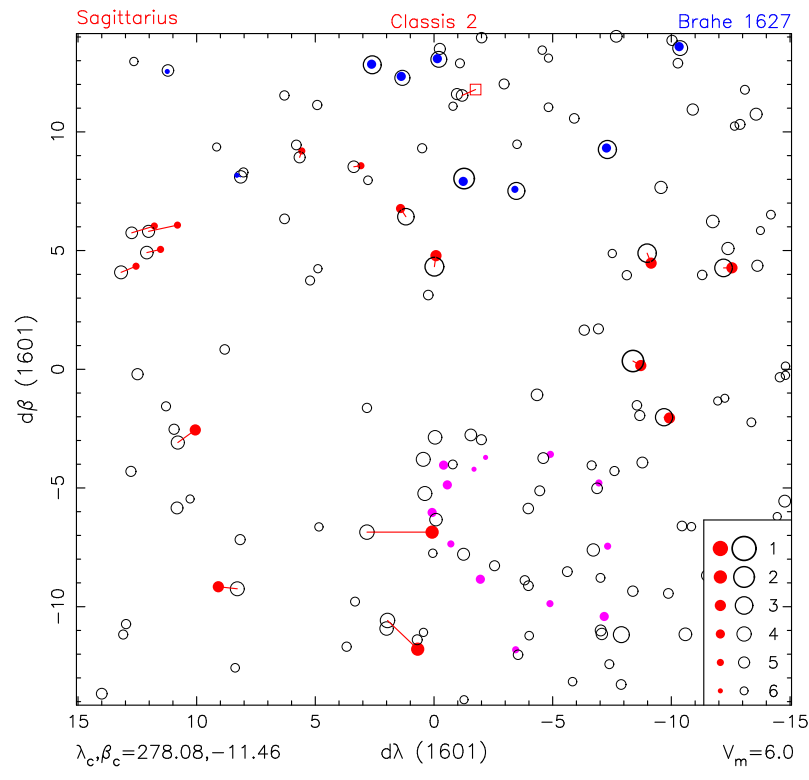

Fig. C.24. Sagittarius.

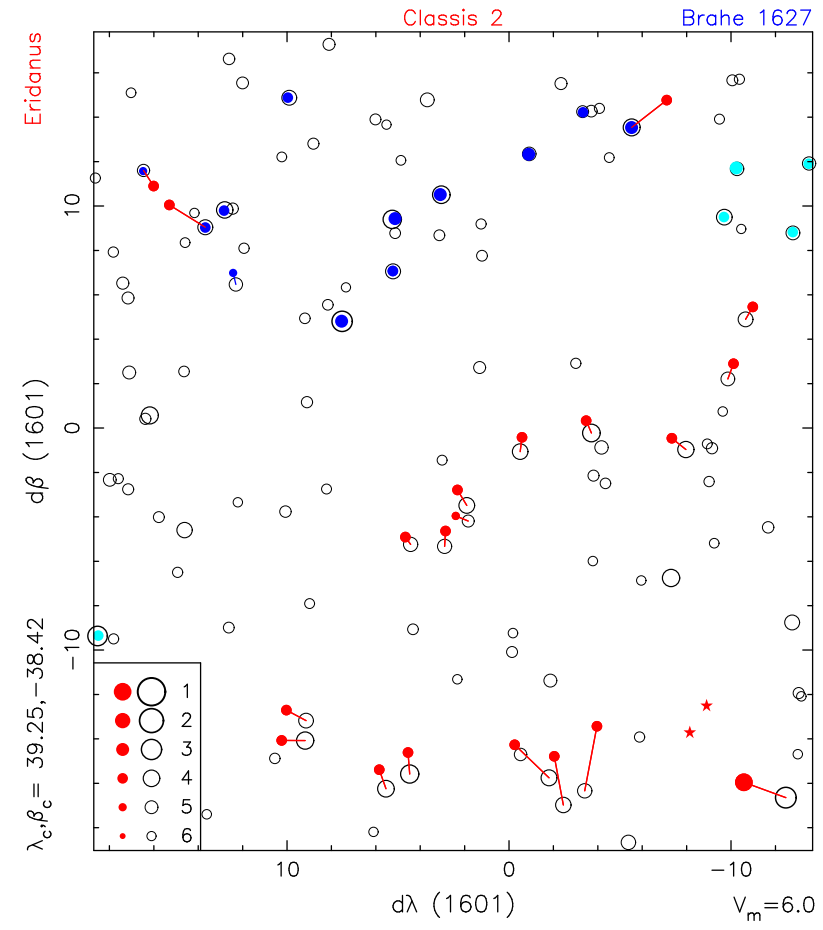

Fig. C.25. Eridanus. The two red stars right below give the positions of K 1139 and K 1140 before emendation.

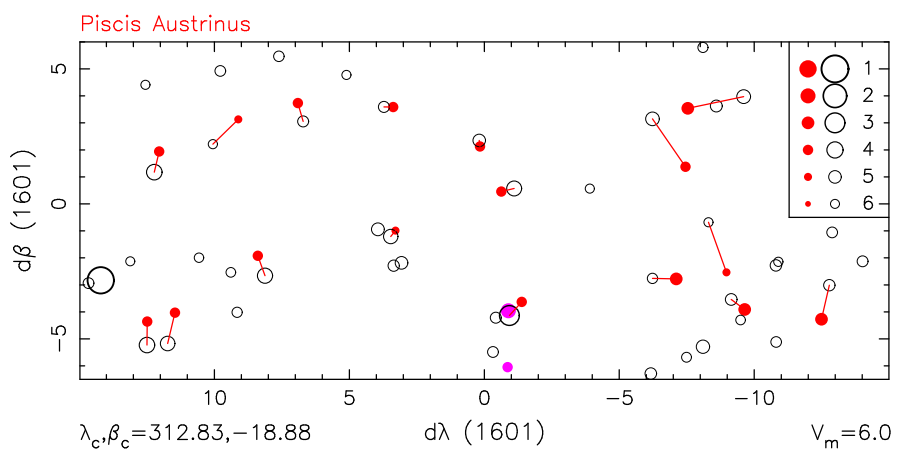

Fig. C.27. Piscis Austrinus. 
Frank Verbunt and Robert H. van Gent: Early star catalogues of the southern sky

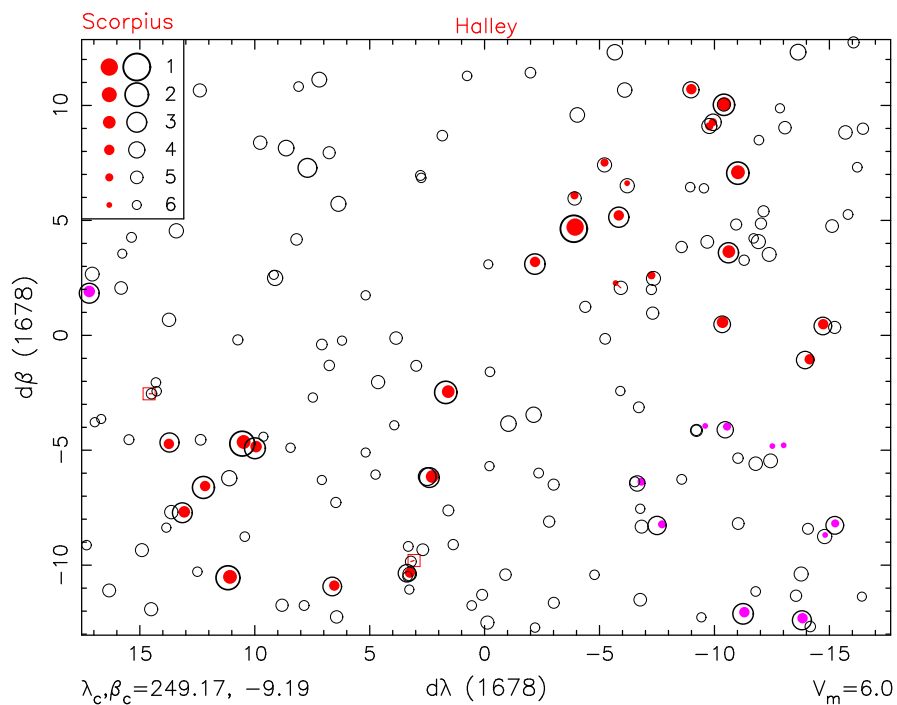

Fig. D.1. Scorpius.

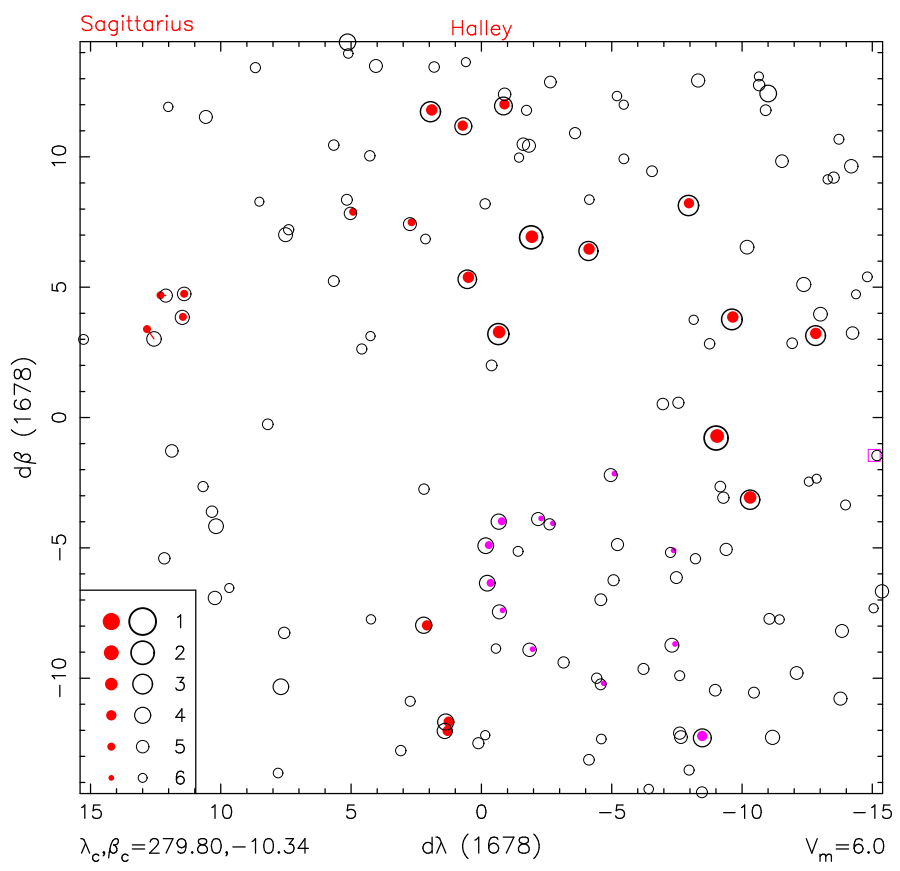

Fig. D.2. Sagittarius.

\section{Appendix D: Constellations in Halley}

The Figures illustrating the identifications by Halley are shown in the rotated ecliptic system.

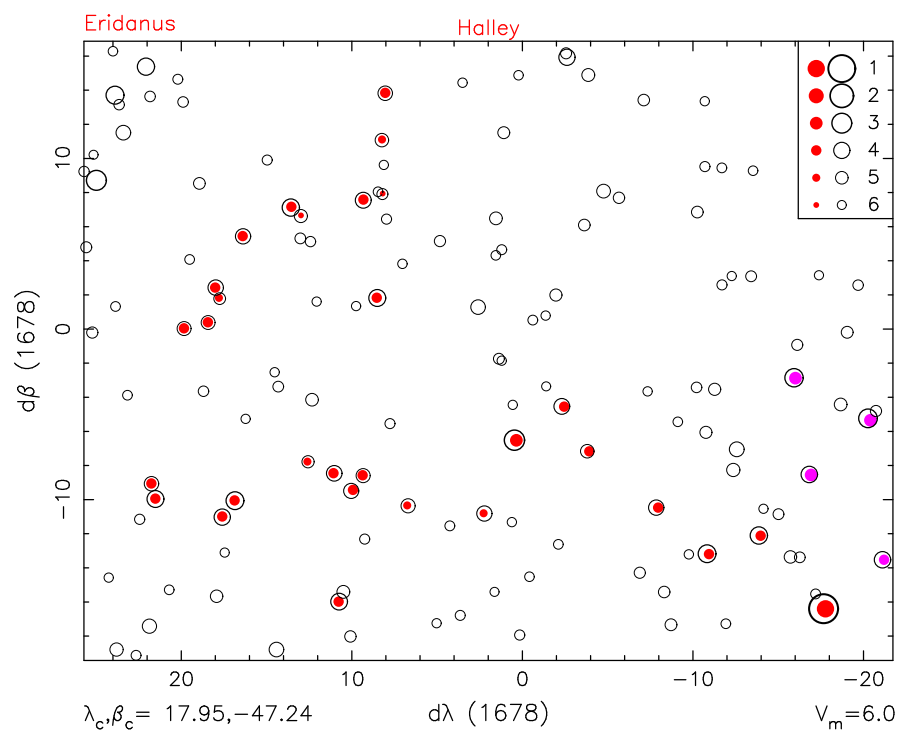

Fig. D.3. Eridanus.

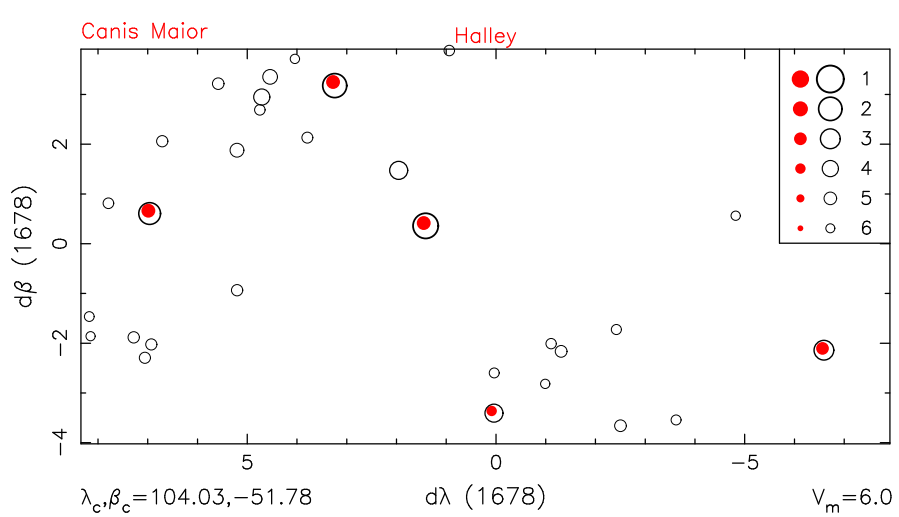

Fig. D.4. Canis Maior in Halley.

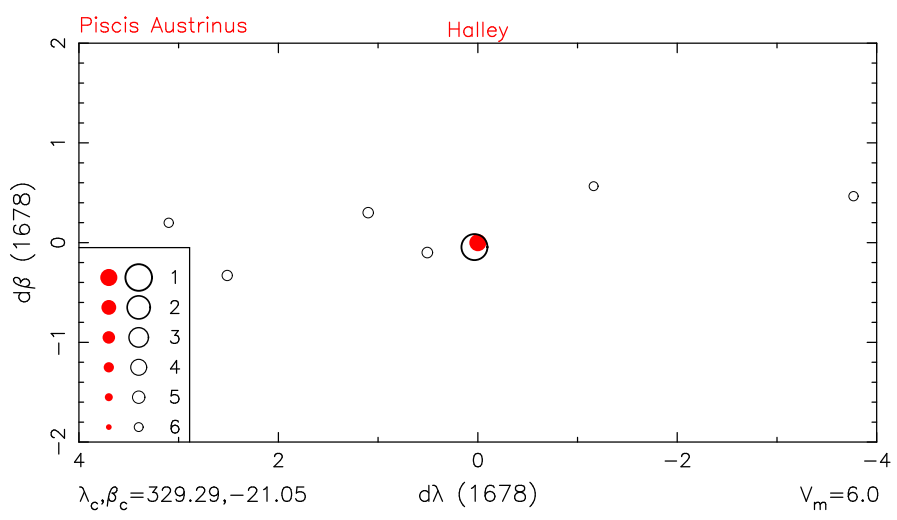

Fig. D.5. Piscis Austrinus. 
Frank Verbunt and Robert H. van Gent: Early star catalogues of the southern sky

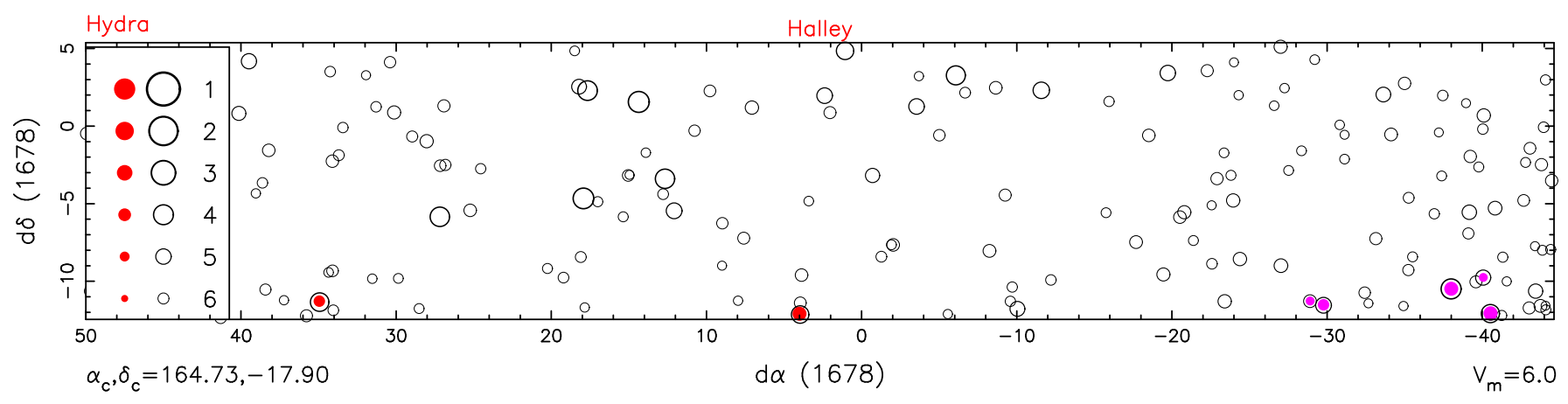

Fig. D.9. Hydra.

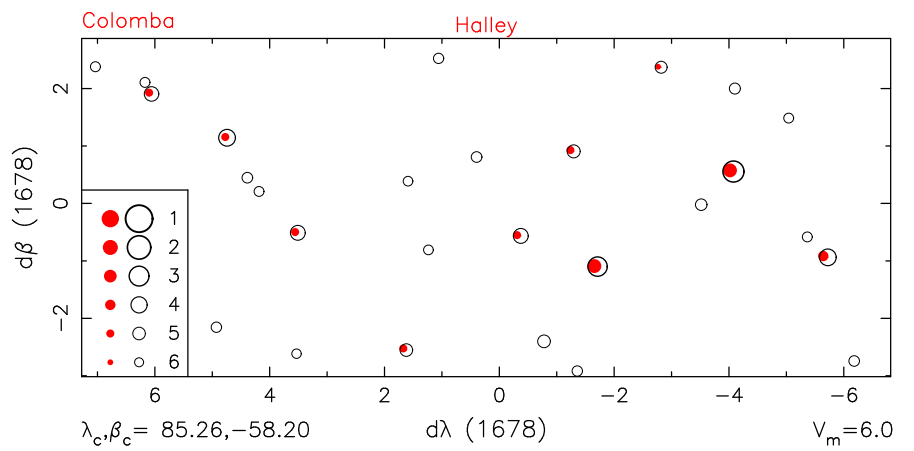

Fig. D.6. Colomba (the dove of Noach).
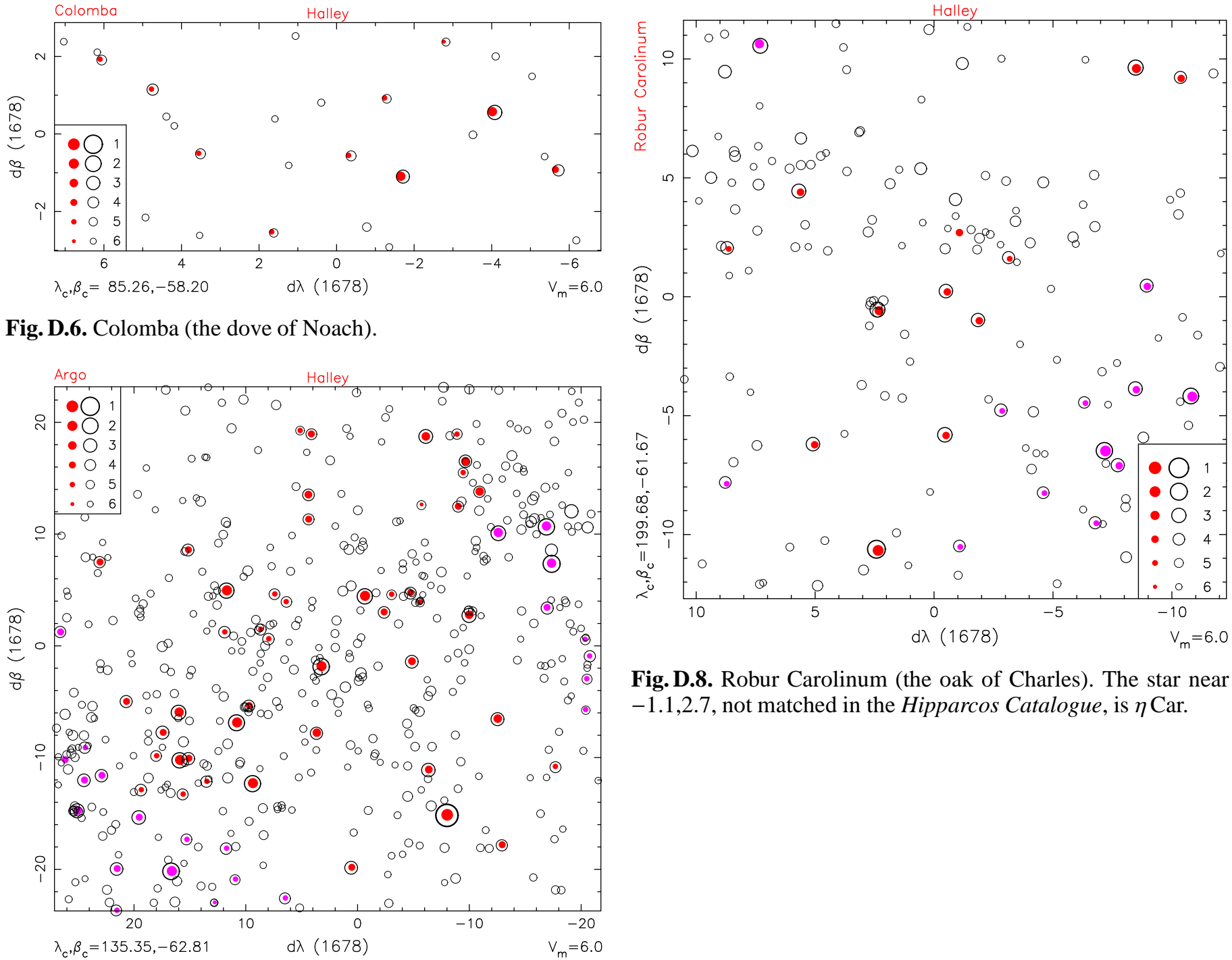

Fig. D.8. Robur Carolinum (the oak of Charles). The star near $-1.1,2.7$, not matched in the Hipparcos Catalogue, is $\eta$ Car.

Fig. D.7. Argo Navis (the ship Argo). 
Frank Verbunt and Robert H. van Gent: Early star catalogues of the southern sky

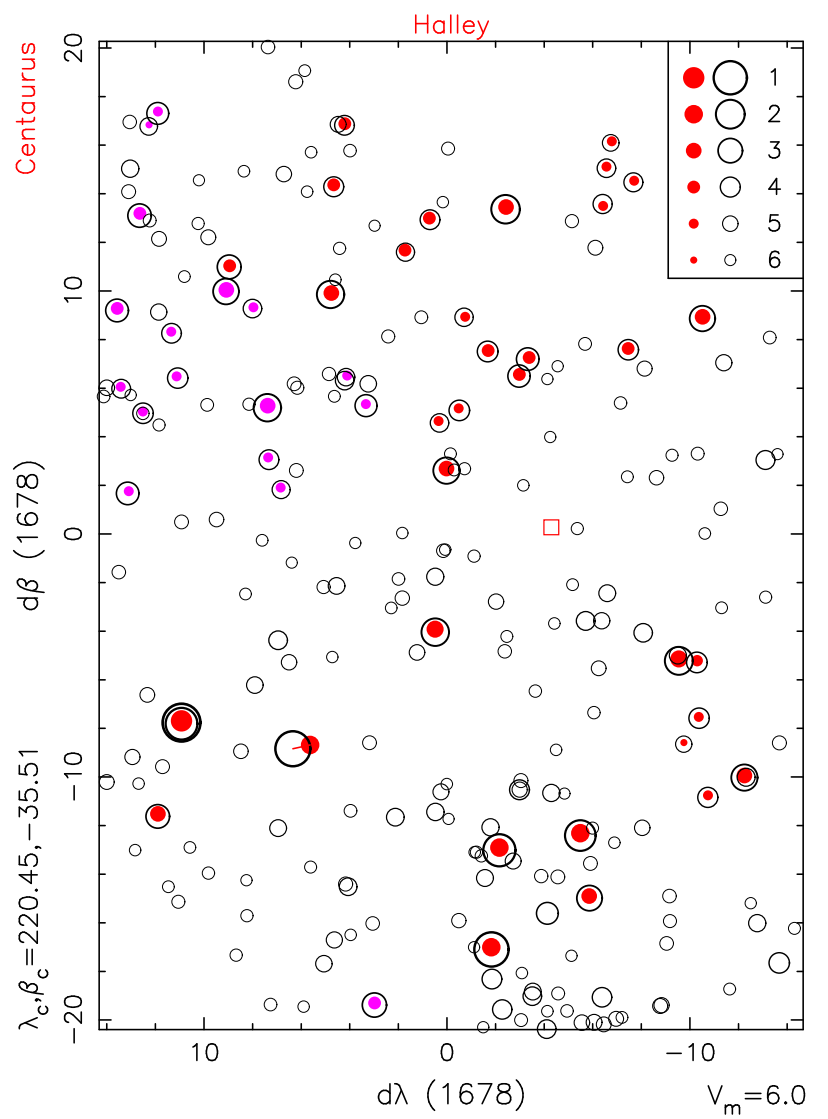

Fig. D.10. Centaurus. The nebulous object is the globular cluster $\omega$ Centauri.

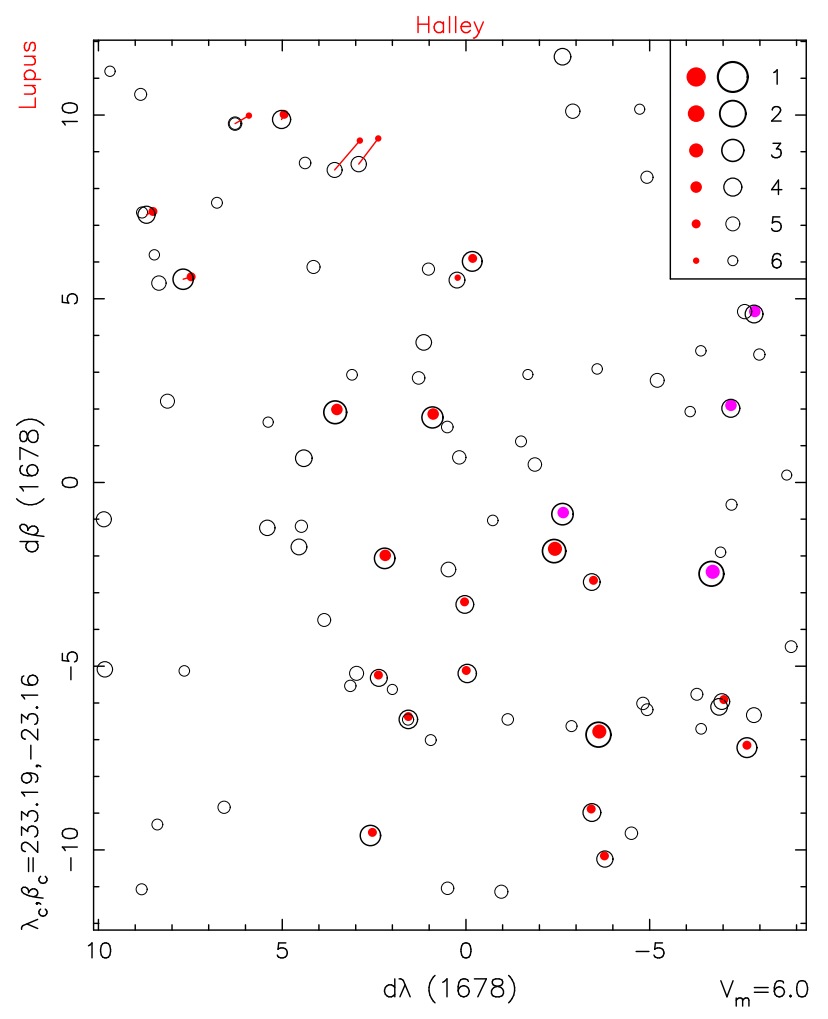

Fig. D.11. Lupus.

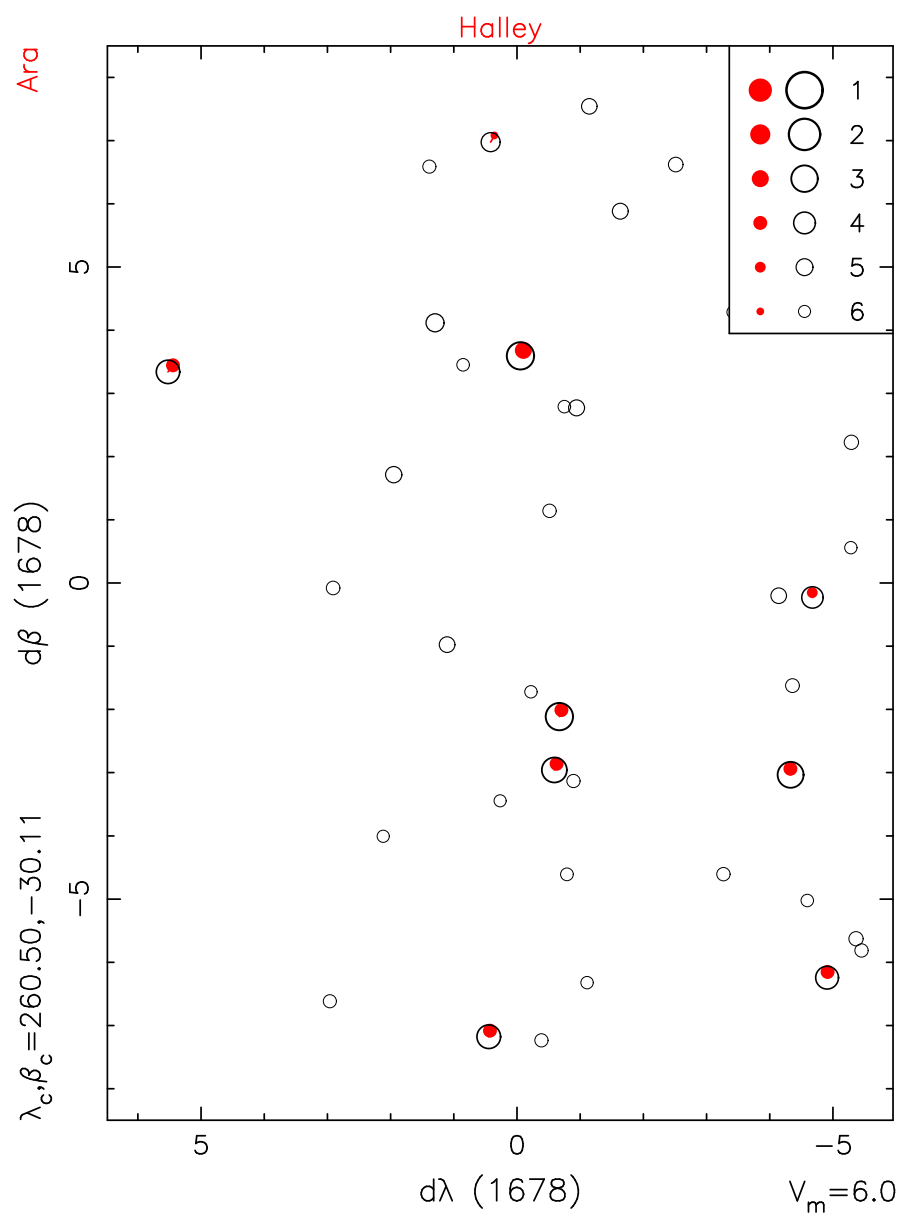

Fig. D.12. Ara, Thuribulum.

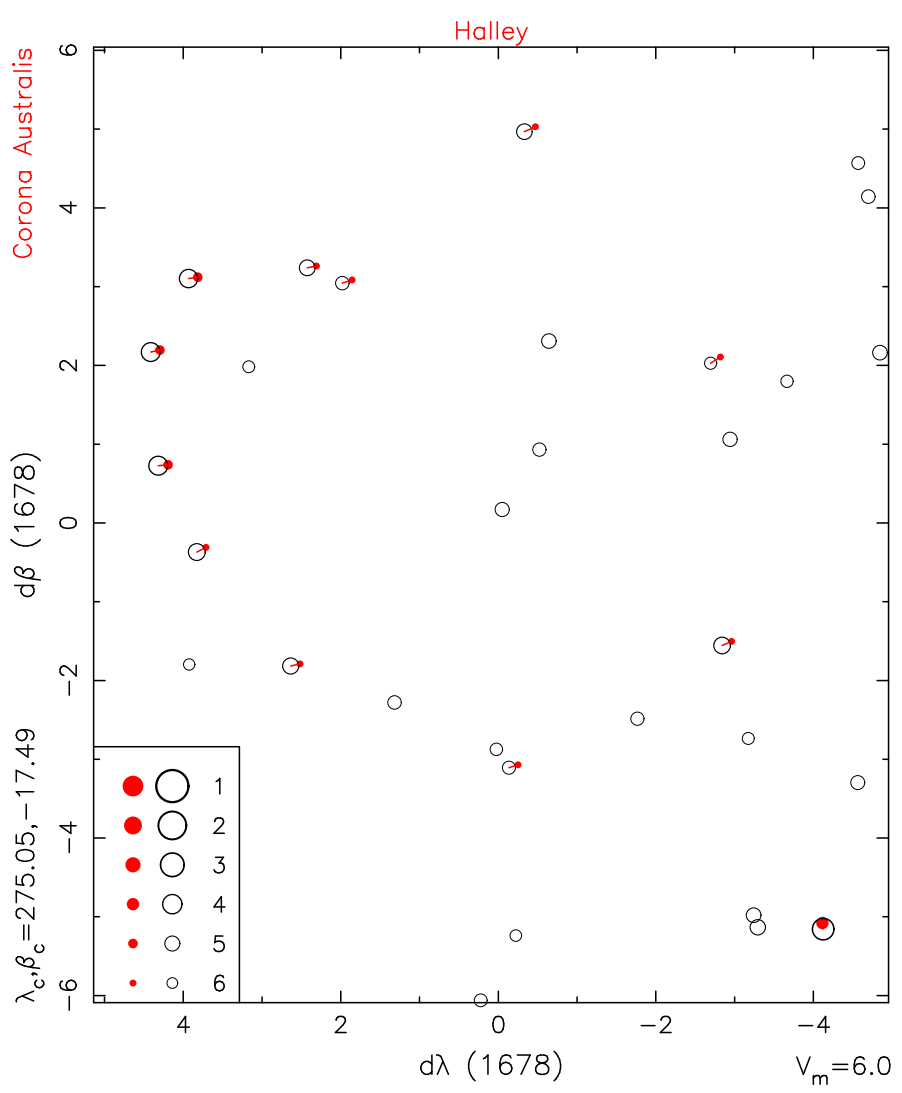

Fig. D.13. Corona Australis. 
Frank Verbunt and Robert H. van Gent: Early star catalogues of the southern sky
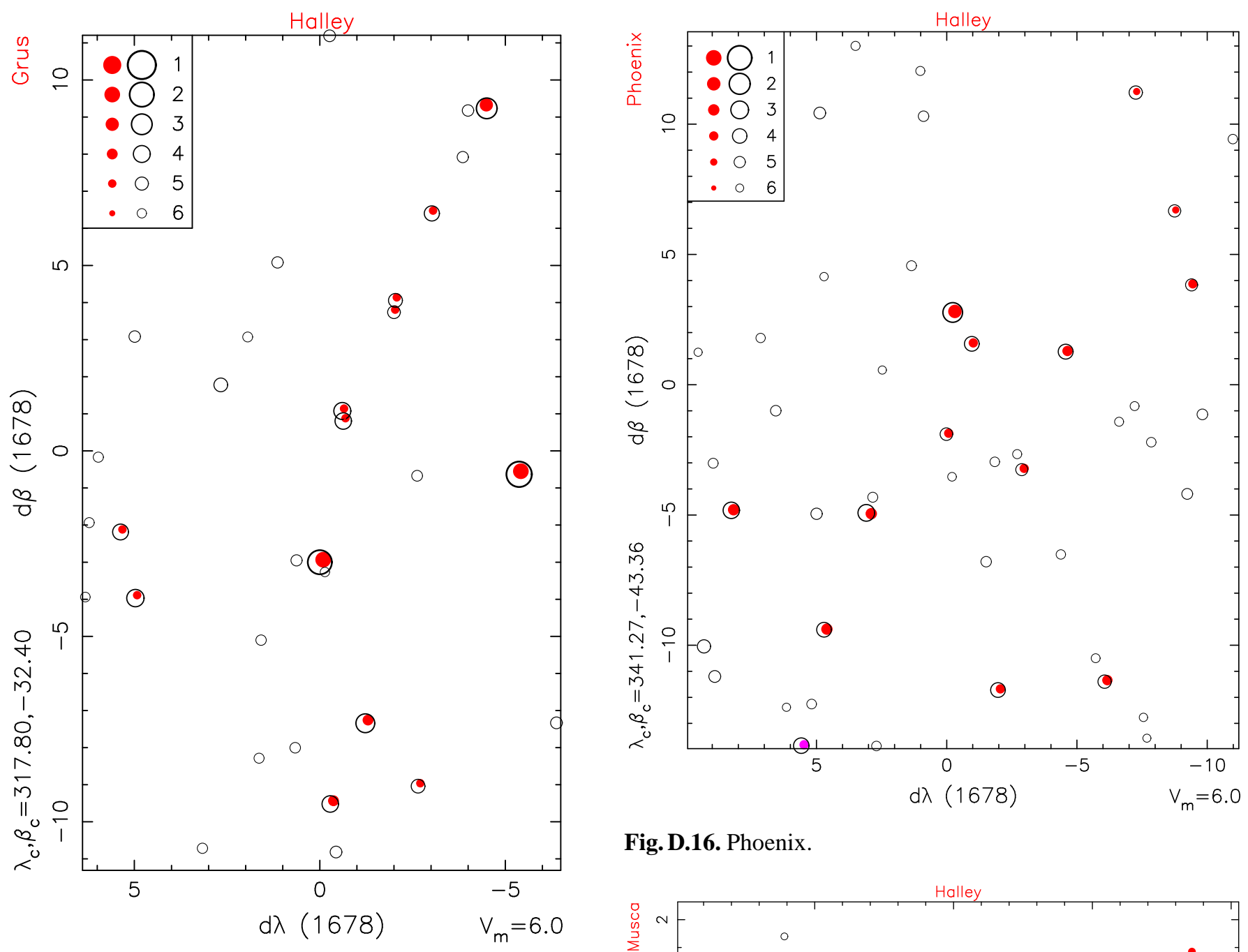

Fig. D.16. Phoenix.

Fig. D.14. Grus.

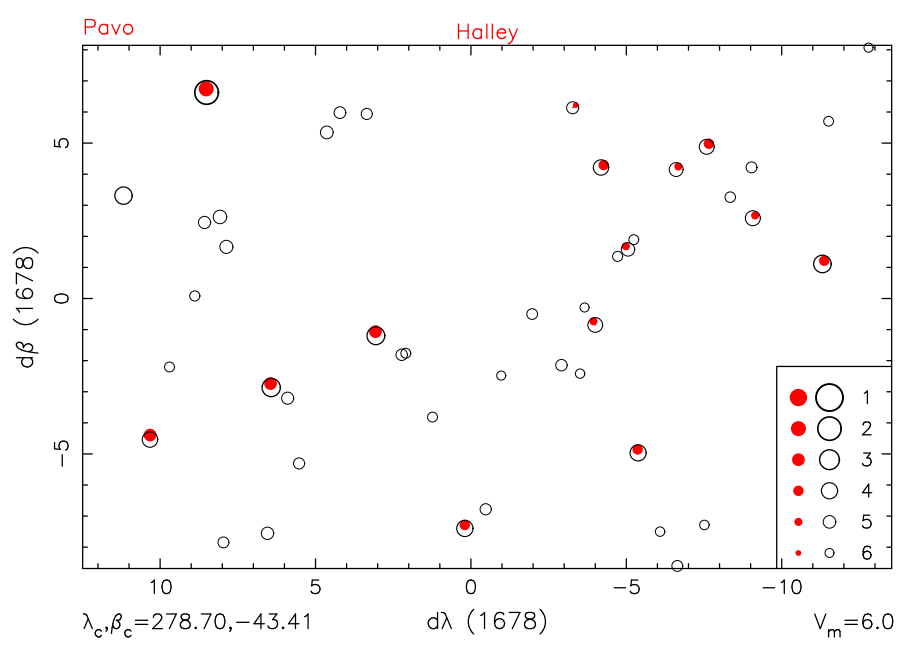

Fig. D.15. Pavo

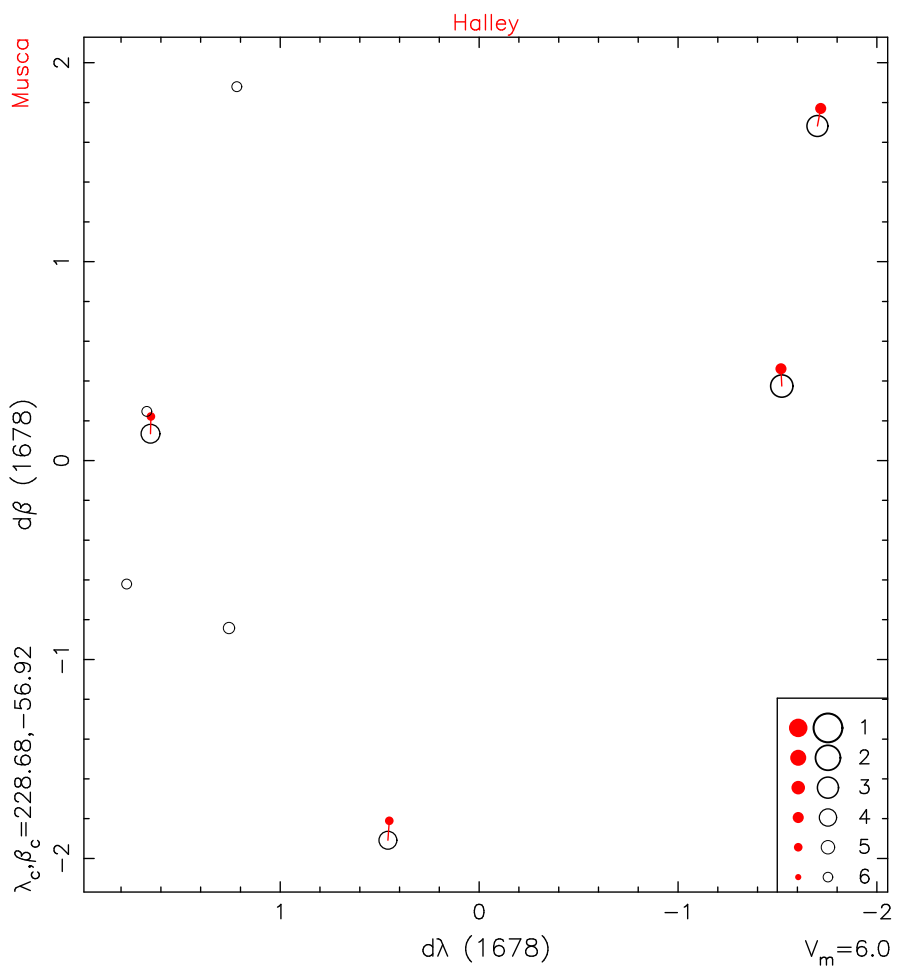

Fig. D.17. Musca Apis (fly, bee). 
Frank Verbunt and Robert H. van Gent: Early star catalogues of the southern sky

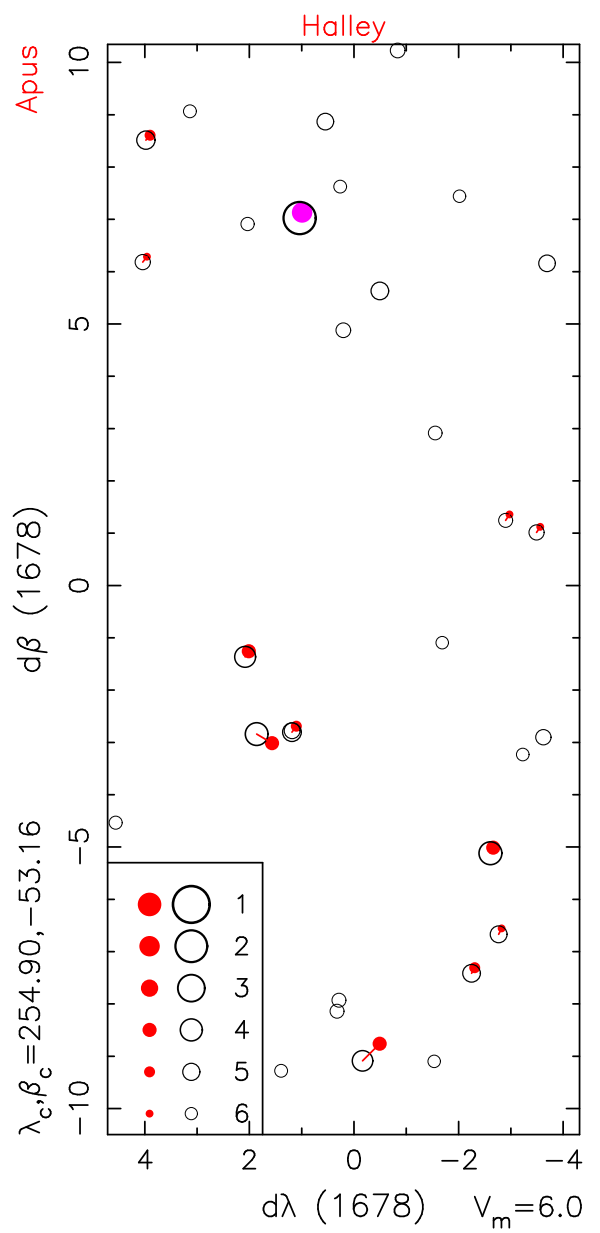

Fig. D.18. Apus Avis, Indica (Indian bird of paradise).

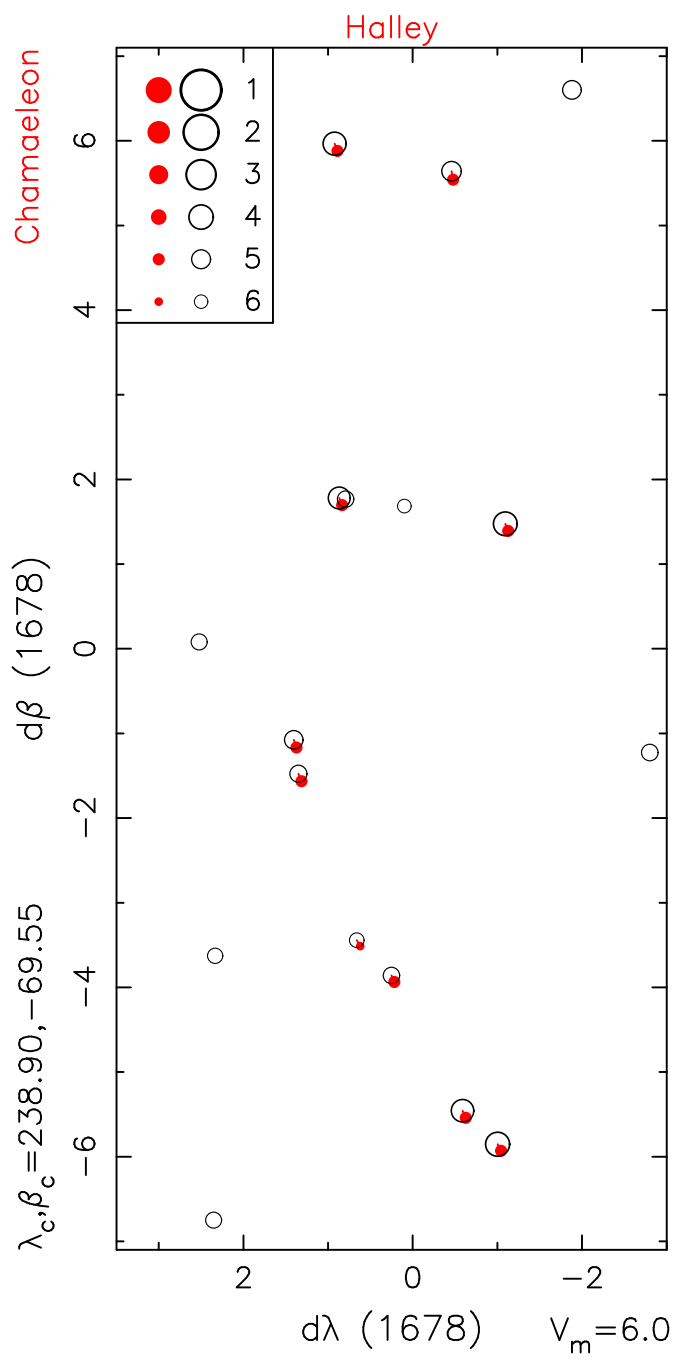

Fig. D.19. Chamaeleon.

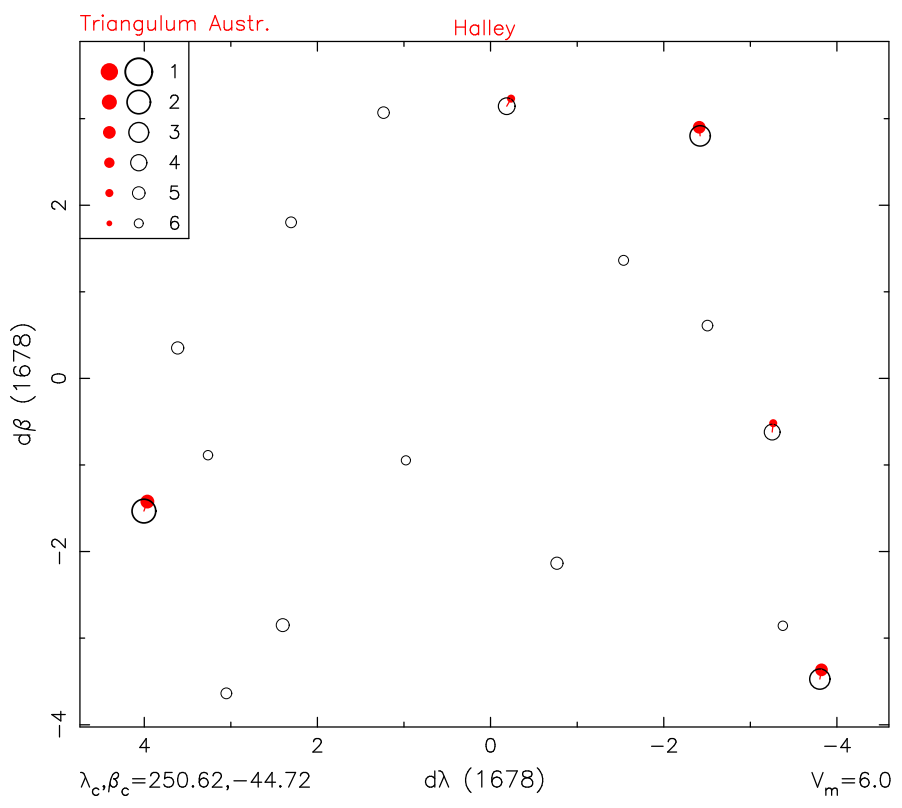

Fig. D.20. Triangulum Australe. 
Frank Verbunt and Robert H. van Gent: Early star catalogues of the southern sky
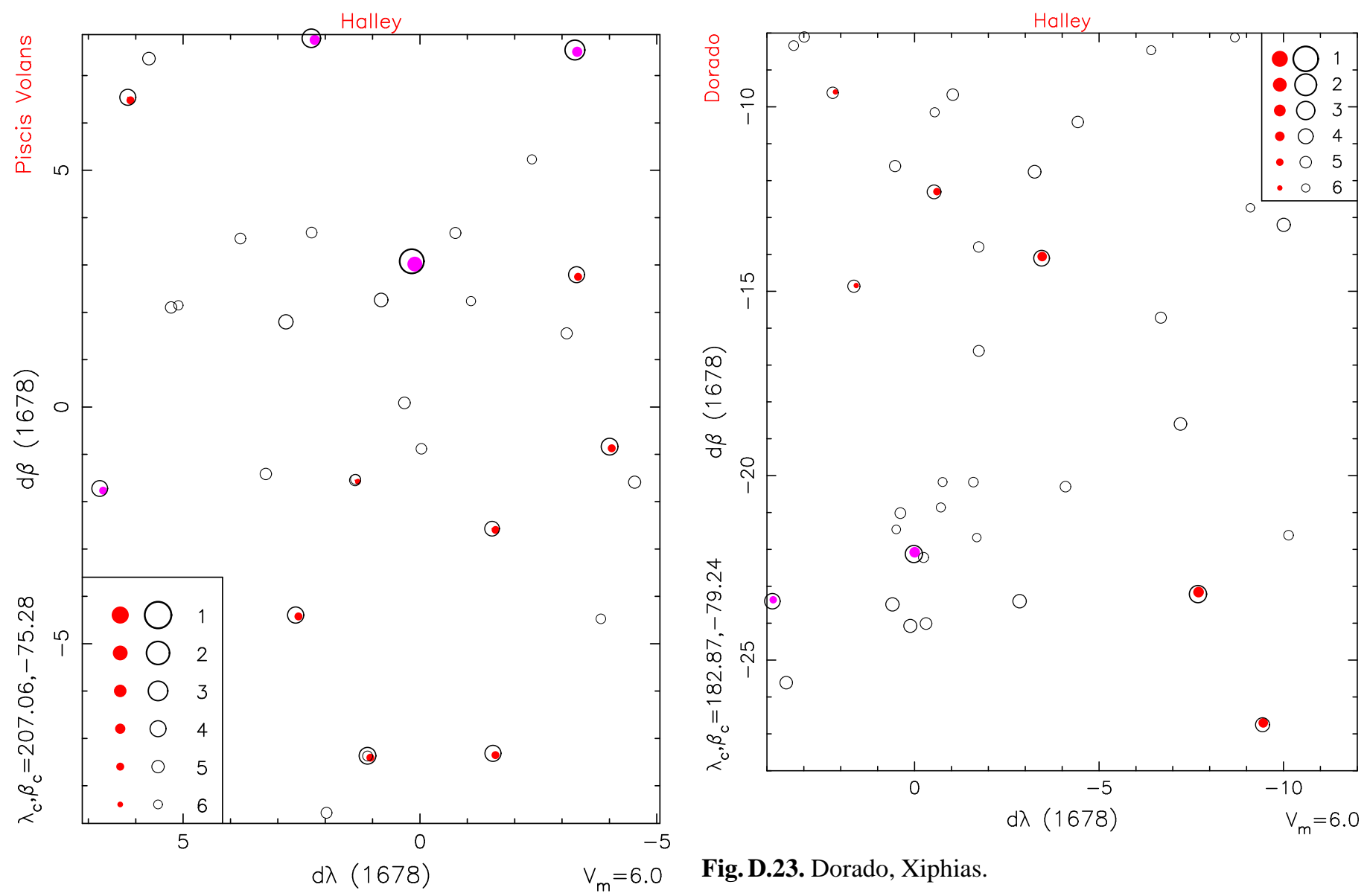

Fig. D.21. Piscis Volans (flying fish).

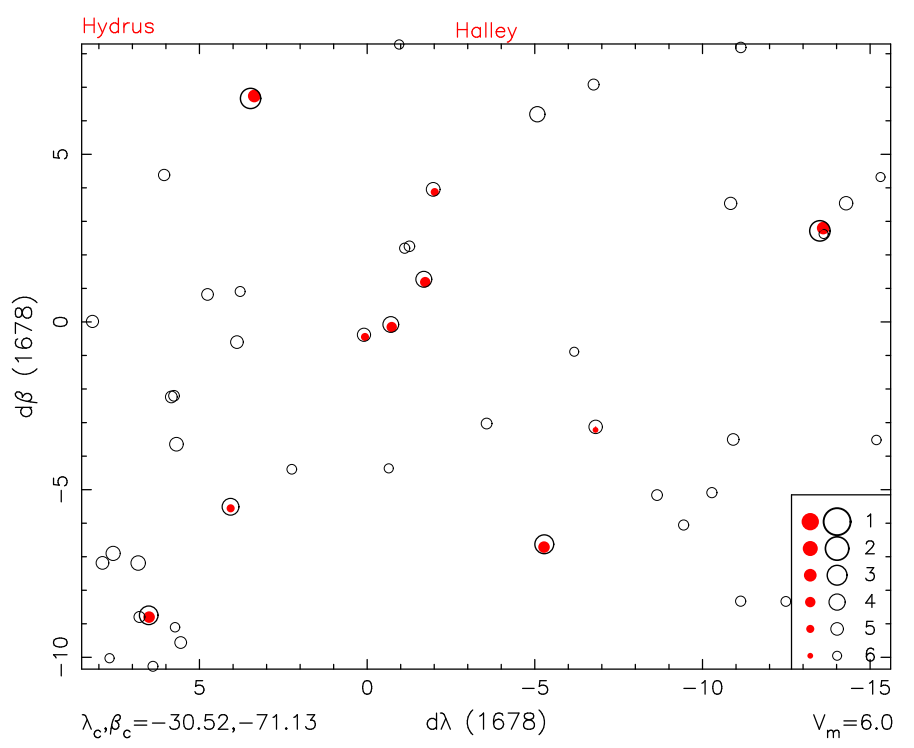

Fig. D.23. Dorado, Xiphias.

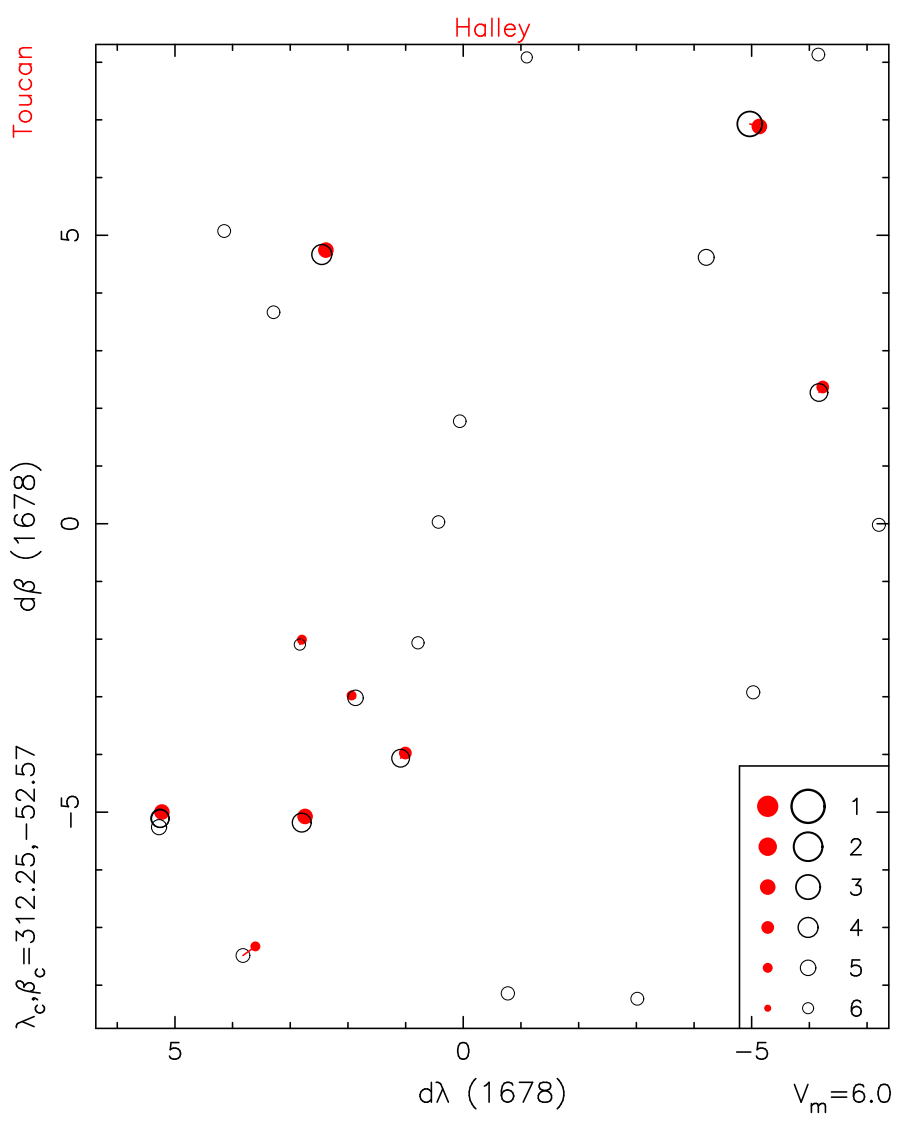

Fig. D.24. Tucana, Anser Americanus (American goose). 